Supporting Information for

\title{
A Diphosphazide-supported Trialkyl Thorium(IV) Complex
}

\author{
Tara K. K. Dickie, Ashraf A. Aborawi and Paul G. Hayes* \\ Department of Chemistry and Biochemistry, University of Lethbridge, Lethbridge, Alberta, T1K \\ 3M4 Canada \\ E-mail: p.hayes@uleth.ca
}

\section{Experimental Section.}

\section{General Considerations.}

Air- and moisture-sensitive materials and reagents were handled under an atmosphere of argon, which was achieved via vacuum line techniques or the use of an MBraun glove box. Solvents used for air-sensitive procedures were purified using an MBraun solvent purification system (SPS), stored in PTFE-sealed glass vessels over sodium benophenone ketyl (THF, dme, pentane, benzene, and toluene), and distilled at the time of use. Benzene- $d_{6}$ and THF- $d_{8}$ was dried over sodium benzophenone ketyl, distilled in vacuo and stored over $4 \AA$ molecular sieves in PTFEsealed glass vessels under argon. All NMR spectra were recorded at ambient temperature with a Bruker Avance II NMR spectrometer (300.13 MHz for ${ }^{1} \mathrm{H}, 75.47 \mathrm{MHz}$ for ${ }^{13} \mathrm{C}\left\{{ }^{1} \mathrm{H}\right\}$, and $121.48 \mathrm{MHz}$ for ${ }^{31} \mathrm{P}\left\{{ }^{1} \mathrm{H}\right\}$ ) or Avance III NMR spectrometer (700.44 MHz for ${ }^{1} \mathrm{H}, 176.13 \mathrm{MHz}$ for ${ }^{13} \mathrm{C}\left\{{ }^{1} \mathrm{H}\right\}$, and 283.54 MHz for $\left.{ }^{31} \mathrm{P}\left\{{ }^{1} \mathrm{H}\right\}\right)$ NMR spectrometer. $\mathrm{H}$-containing species, such as $\mathrm{C}_{6} \mathrm{D}_{5} \mathrm{H}\left(\delta 7.16\left({ }^{1} \mathrm{H}\right), \delta\right.$ $\left.128.39\left({ }^{13} \mathrm{C}\right)\right)$ and $d_{7}$-THF $\left(\left(\delta 1.73\right.\right.$ and $\delta 3.58\left({ }^{1} \mathrm{H}\right), \delta 25.37$ and $\left.\delta 67.57\left({ }^{13} \mathrm{C}\right)\right)$ were used as internal references $\left(\delta 7.16\left({ }^{1} \mathrm{H}\right), \delta 128.39\left({ }^{13} \mathrm{C}\right)\right) .(\mathrm{s}=$ singlet, $\mathrm{d}=$ doublet, $\mathrm{t}=$ triplet, $\mathrm{q}=$ quartet, quint $=$ quintet, $\mathrm{sp}=$ septet, $\mathrm{m}=$ multiplet, $\mathrm{br}=$ broad, ov = overlapping signals, $\mathrm{dd}=$ doublet of doublet). 
Assignments of resonances were supplemented by ${ }^{1} \mathrm{H}-{ }^{1} \mathrm{H}$ COSY, ${ }^{13} \mathrm{C}\left\{{ }^{1} \mathrm{H}\right\} \mathrm{APT},{ }^{1} \mathrm{H}-{ }^{13} \mathrm{C}\left\{{ }^{1} \mathrm{H}\right\}$, and HSQC/HMBC experiments. The compound 4-isopropylphenyl (Pipp) azide was prepared according to our previously reported synthesis ${ }^{1}$ and dried over $4 \AA$ sieves as a solution in toluene. $\mathrm{ThCl}_{4}(\mathrm{dme})_{2}$ was prepared according to Kiplinger et al. ${ }^{2}$ from Th$\left(\mathrm{NO}_{3}\right)_{4}\left(\mathrm{H}_{2} \mathrm{O}\right)_{5}$. $\mathrm{LiCH}_{2} \mathrm{SiMe}_{3}$ was purchased as a $1.0 \mathrm{M}$ solution in pentane from Sigma Aldrich; the solvent was removed in vacuo and the compound used as a solid. All other reagents were purchased from commercial sources and used as received.

\section{Synthesis and Characterization of Compounds.}

Synthesis of 2,5-bis(diisopropylphosphino)-N-K-pyrrole, (1).

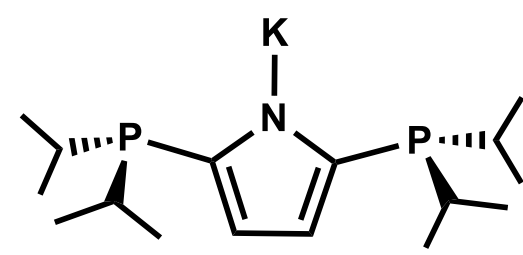

1

In an inert atmosphere glove box, 2,5- $\left(\mathrm{P}^{i} \mathrm{Pr}_{2}\right)_{2} \mathrm{NH}\left(\mathrm{C}_{4} \mathrm{H}_{2}\right)(49.8 \mathrm{mg}$, $0.167 \mathrm{mmol})^{3}$ and potassium hydride $(9.7 \mathrm{mg}, 0.24 \mathrm{mmol}, 1.4$ equiv) were added to a $100 \mathrm{~mL}$ RBF with a Teflon ${ }^{\circledR}$ coated stir

bar. The reaction vessel was attached to a double manifold vacuum line and $10 \mathrm{~mL}$ of THF was transferred to the flask at $-78{ }^{\circ} \mathrm{C}$. Evolution of $\mathrm{H}_{2}$ gas was observed upon gradual warming to ambient temperature. The reaction mixture was then heated while stirring under argon at $45^{\circ} \mathrm{C}$ for 2 hours. The solvent was removed in vacuo leaving an off-white solid. The product was extracted into benzene $(1 \times 1.5 \mathrm{~mL})$ and the solution was filtered through Celite ${ }^{\circledR}$ to remove excess KH. After filtration, benzene was removed in vacuo, affording a white solid. Yield: $42.1 \mathrm{mg}$, 74.6\%. ${ }^{1} \mathrm{H}$ NMR $\left(\mathrm{C}_{6} \mathrm{D}_{6}, 300.13 \mathrm{MHz}\right): \delta 1.16\left(\mathrm{~m}, 24 \mathrm{H}, \mathrm{CH}\left(\mathrm{CH}_{3}\right)_{2}\right), 2.06\left(\mathrm{sp},{ }^{3} \mathrm{~J}_{\mathrm{HH}}=6.7 \mathrm{~Hz}, 4 \mathrm{H}\right.$, $\left.\mathrm{CH}\left(\mathrm{CH}_{3}\right)_{2}\right), 6.70(\mathrm{~s}, 2 \mathrm{H}$, pyrrole- $\mathrm{CH}) .{ }^{13} \mathrm{C}\left\{{ }^{1} \mathrm{H}\right\} \operatorname{NMR}\left(\mathrm{C}_{6} \mathrm{D}_{6}, 75.47 \mathrm{MHz}\right): \delta 20.2\left(\mathrm{~d},{ }^{2} \mathrm{~J}_{\mathrm{CP}}=6.7 \mathrm{~Hz}\right.$, $\left.\mathrm{CH}\left(\mathrm{CH}_{3}\right)_{2}\right), 21.4\left(\mathrm{~d},{ }^{1} \mathrm{~J}_{\mathrm{CP}}=19.0 \mathrm{~Hz}, \mathrm{CH}\left(\mathrm{CH}_{3}\right)_{2}\right), 24.6\left(\mathrm{~d},{ }^{2} \mathrm{~J}_{\mathrm{CP}}=9.1 \mathrm{~Hz}, \mathrm{CH}\left(\mathrm{CH}_{3}\right)_{2}\right), 115.5(\mathrm{~s}$, 3,4-pyrrole 
$\mathrm{CH}), 135.02\left(\mathrm{dd},{ }^{1} \mathrm{~J}_{\mathrm{CP}}=15.9 \mathrm{~Hz},{ }^{3} \mathrm{~J}_{\mathrm{CP}}=12.6,2,5-\right.$ pyrrole $\left.C\right) .{ }^{31} \mathrm{P}\left\{{ }^{1} \mathrm{H}\right\} \mathrm{NMR}\left(\mathrm{C}_{6} \mathrm{D}_{6}, 121.49 \mathrm{MHz}\right): \delta-4.9$

(s). Anal. Calcd. (\%) for $\mathrm{C}_{16} \mathrm{H}_{30} \mathrm{KNP}_{2}$ : C: 56.95; H: 8.96; N: 4.15. Found: C: 56.98; H: 8.97; N: 4.30 .

\section{Synthesis of $K L_{P=N 3},(2)$.}

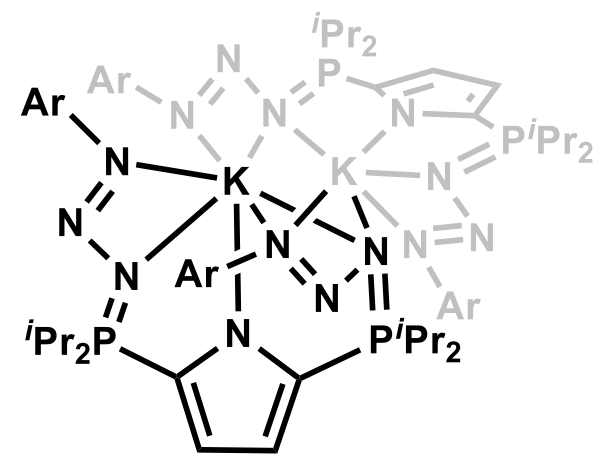

2

In an inert atmosphere glove box, compound $\mathbf{1}(175.3 \mathrm{mg}$, $0.5195 \mathrm{mmol}$ ) was added into a $20 \mathrm{~mL}$ scintillation vial and dissolved in toluene (3 mL). Pipp azide (167.0 mg, 1.036 mmol, 2 equiv) diluted in toluene $(1 \mathrm{~mL})$ was added dropwise to the vial containing $\mathbf{1}$, resulting in a clear dark yellow solution. The solution was stirred for two minutes with a Teflon ${ }^{\circledR}$ coated stir bar and the solvent was removed in vacuo. The product was washed with pentane $(3 \times 2 \mathrm{~mL})$ and dried in vacuo resulting in a pale yellow solid. Yield: $0.1753 \mathrm{~g}, 61.25 \% .{ }^{1} \mathrm{H}$ NMR $\left(\mathrm{C}_{6} \mathrm{D}_{6}, 300.13 \mathrm{MHz}\right): \delta 1.02$ $\left(\mathrm{dd},{ }^{3} \mathrm{~J}_{\mathrm{HP}}=14.9 \mathrm{~Hz},{ }^{3} \mathrm{~J}_{\mathrm{HH}}=6.9 \mathrm{~Hz}, 24 \mathrm{H}, \mathrm{P}-\mathrm{CH}\left(\mathrm{CH}_{3}\right)_{2}\right), 1.23\left(\mathrm{~d},{ }^{3} \mathrm{~J}_{\mathrm{HH}}=6.9 \mathrm{~Hz}, 12 \mathrm{H}, \mathrm{Ar}-\mathrm{CH}\left(\mathrm{CH}_{3}\right)_{2}\right), 2.44$ $\left(\mathrm{dsp},{ }^{3} \mathrm{~J}_{\mathrm{HH}}=6.9 \mathrm{~Hz},{ }^{2} \mathrm{~J}_{\mathrm{HP}}=2.4 \mathrm{~Hz}, 4 \mathrm{H}, \mathrm{P}-\mathrm{CH}\left(\mathrm{CH}_{3}\right)_{2}\right), 2.81\left(\mathrm{sp},{ }^{3} \mathrm{~J}_{\mathrm{HH}}=6.9 \mathrm{~Hz}, 2 \mathrm{H}, \mathrm{Ar}-\mathrm{CH}\left(\mathrm{CH}_{3}\right)_{2}\right), 6.88$ $\left(d,{ }^{3} \mathrm{JP}_{\mathrm{HP}}=2.2 \mathrm{~Hz}, 2 \mathrm{H}\right.$, pyrrole-CH), $7.19\left(\mathrm{~d},{ }^{3} \mathrm{~J}_{\mathrm{HH}}=8.4 \mathrm{~Hz}, 4 \mathrm{H}\right.$, meta-aromatic $\left.\mathrm{CH}\right), 7.59\left(\mathrm{~d},{ }^{3} \mathrm{~J}_{\mathrm{HH}}=8.4\right.$ $\mathrm{Hz} 4 \mathrm{H}$, ortho-aromatic $\mathrm{CH}) .{ }^{13} \mathrm{C}\left\{{ }^{1} \mathrm{H}\right\} \mathrm{NMR}\left(\mathrm{C}_{6} \mathrm{D}_{6}, 75.47 \mathrm{MHz}\right): \delta 16.0\left(\mathrm{~d},{ }^{2} \mathrm{~J}_{\mathrm{CP}}=2.8 \mathrm{~Hz}, \mathrm{P}-\mathrm{CH}\left(\mathrm{CH}_{3}\right)_{2}\right)$, $16.8\left(\mathrm{~d},{ }^{2} \mathrm{~J}_{\mathrm{CP}}=1.9 \mathrm{~Hz}, \mathrm{P}-\mathrm{CH}\left(\mathrm{CH}_{3}\right)_{2}\right), 24.2\left(\mathrm{~d},{ }^{1} \mathrm{~J}_{\mathrm{CP}}=54.8 \mathrm{~Hz}, \mathrm{P}-\mathrm{CH}\left(\mathrm{CH}_{3}\right)_{2}\right), 24.8\left(\mathrm{~s}, \mathrm{Ar}-\mathrm{CH}\left(\mathrm{CH}_{3}\right)_{2}\right), 34.5$ (s, $\left.\mathrm{Ar}-\mathrm{CH}\left(\mathrm{CH}_{3}\right)_{2}\right), 118.0\left(\mathrm{dd}^{2}{ }^{2} \mathrm{~J}_{\mathrm{CP}}=27.5 \mathrm{~Hz},{ }^{3} \mathrm{~J}_{\mathrm{CP}}=10.5 \mathrm{~Hz}, 3,4-\right.$ pyrrole $\left.\mathrm{CH}\right), 121.5$ (s, aromatic $\mathrm{CH}$ ), $127.3(\mathrm{~s}$, aromatic $C H), 125.0\left(\mathrm{dd},{ }^{1} \mathrm{~J}_{\mathrm{CP}}=134.1 \mathrm{~Hz},{ }^{3} \mathrm{~J}_{\mathrm{CP}}=20.8 \mathrm{~Hz}, 2,5\right.$-pyrrole $\left.C\right), 145.7$ (s, aromatic ipso-C), 152.04 (s, aromatic ipso-C). ${ }^{31} \mathrm{P}\left\{{ }^{1} \mathrm{H}\right\} \operatorname{NMR}\left(\mathrm{C}_{6} \mathrm{D}_{6}, 121.49 \mathrm{MHz}\right): \delta 44.7$ (s). Anal. Calcd. (\%) for $\mathrm{C}_{34} \mathrm{H}_{52} \mathrm{KN}_{7} \mathrm{P}_{2}: \mathrm{C}: 61.89 ; \mathrm{H}: 7.94 ; \mathrm{N}: 14.86$. Found: $\mathrm{C}: 61.85 ; \mathrm{H}: 7.98 ; \mathrm{N}: 14.76$. 


\section{Synthesis of $\mathrm{LP}_{\mathrm{P}=\mathrm{N} 3} \mathrm{ThCl}_{3}(3)$.}

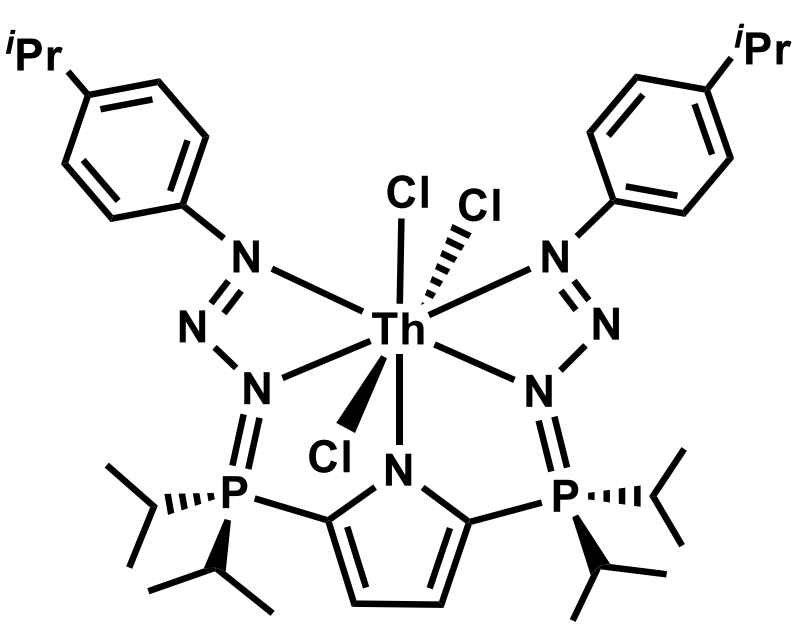

3

In an inert atmosphere glove box, compound $\mathbf{2}$ (31.2 $\mathrm{mg}, 0.0455 \mathrm{mmol})$ and $\mathrm{ThCl}_{4}(\mathrm{dme})_{2}(26.1$ $\mathrm{mg}, 0.0455 \mathrm{mmol}$ ) were weighed into a $20 \mathrm{~mL}$ scintillation vial with a Teflon ${ }^{\circledR}$ coated stir bar. THF cooled to $-35{ }^{\circ} \mathrm{C}$ was added $(3 \mathrm{~mL})$ to the vial and the cloudy solution was kept cold (-35 ${ }^{\circ} \mathrm{C}$ ) and stirred with a Teflon ${ }^{\circledR}$ coated stir bar for

5 minutes. The solution was then quickly filtered through Celite ${ }^{\circledR}$ to remove salts and the THF was removed in vacuo. The product was washed with pentane $(3 \times 3 \mathrm{~mL})$ and dried in vacuo. The resulting product was an off-white solid. Yield: $38.6 \mathrm{mg}, 85.1 \% .{ }^{1} \mathrm{H}$ NMR (THF- $\left.d_{8}, 300.13 \mathrm{MHz}\right): \delta$ $1.27\left(\mathrm{~d},{ }^{3} \mathrm{~J}_{\mathrm{HH}}=6.9 \mathrm{~Hz}, 12 \mathrm{H}, \mathrm{Ar}-\mathrm{CH}\left(\mathrm{CH}_{3}\right)_{2}\right), 1.34\left(\mathrm{~m}, 24 \mathrm{H}, \mathrm{P}-\mathrm{CH}\left(\mathrm{CH}_{3}\right)_{2}\right), 2.97$ (ov sp and dsp, 6H, P$\mathrm{CH}\left(\mathrm{CH}_{3}\right)_{2}$ and $\left.\mathrm{Ar}-\mathrm{CH}\left(\mathrm{CH}_{3}\right)_{2}\right), 7.0\left(\mathrm{dd},{ }^{2} \mathrm{~J}_{\mathrm{HP}}=2.4 \mathrm{~Hz},{ }^{3} J_{\mathrm{HP}}=1.2 \mathrm{~Hz}, 2 \mathrm{H}\right.$, pyrrole-CH), $7.27\left(\mathrm{~d},{ }^{3} \mathrm{~J}_{\mathrm{HH}}=8.4\right.$ $\mathrm{Hz}, 4 \mathrm{H}$, meta-aromatic $\mathrm{CH}), 7.93\left(\mathrm{~d},{ }^{3} \mathrm{~J}_{\mathrm{HH}}=8.4 \mathrm{~Hz}, 4 \mathrm{H}\right.$, ortho-aromatic $\left.\mathrm{CH}\right) .{ }^{13} \mathrm{C}\left\{{ }^{1} \mathrm{H}\right\} \mathrm{NMR}\left(\mathrm{C}_{6} \mathrm{D}_{6}\right.$, $75.47 \mathrm{MHz}$ ): $\delta 15.9\left(\mathrm{~s}, \mathrm{P}-\mathrm{CH}\left(\mathrm{CH}_{3}\right)_{2}\right), 16.2\left(\mathrm{~s}, \mathrm{P}-\mathrm{CH}\left(\mathrm{CH}_{3}\right)_{2}\right), 24.4\left(\mathrm{~s}, \mathrm{Ar}-\mathrm{CH}\left(\mathrm{CH}_{3}\right)_{2}\right), 26.0\left(\mathrm{~d},{ }^{1} J_{\mathrm{CP}}=50.7\right.$ $\left.\mathrm{Hz}, \mathrm{P}-\mathrm{CH}\left(\mathrm{CH}_{3}\right)_{2}\right), 34.6$ (s, $\left.\mathrm{Ar}-\mathrm{CH}\left(\mathrm{CH}_{3}\right)_{2}\right), 118.8$ (s, 3,4-pyrrole $\left.\mathrm{CH}\right), 123.0$ (s, aromatic $\left.\mathrm{CH}\right), 126.6$ (d, ${ }^{1} J_{\mathrm{CP}}=15.7 \mathrm{~Hz}, 2,5-$ pyrrole $C$ ), 127.5 (s, aromatic $C \mathrm{H}$ ), 147.4 (s, aromatic ipso-C), 149.8 (s, aromatic ipso-C). ${ }^{31} \mathrm{P}\left\{{ }^{1} \mathrm{H}\right\}$ NMR $\left(\mathrm{C}_{6} \mathrm{D}_{6}, 121.49 \mathrm{MHz}\right): \delta 63.0$ (s). Anal. Calcd. (\%) for $\mathrm{C}_{34} \mathrm{H}_{52} \mathrm{Cl}_{3} \mathrm{~N}_{7} \mathrm{P} 2 \mathrm{Th}$ : C: 42.58; H: 5.46; N: 10.22. Found: C: 41.81; H: 5.04; N: 10.02. 


\section{Synthesis of $\mathrm{LP}_{\mathrm{P}=\mathrm{N} 3} \mathrm{Th}\left(\mathrm{CH}_{2} \mathrm{SiMe}_{3}\right)_{3} \bullet \mathrm{LiCl},(4 \bullet \mathrm{LiCl})$.}

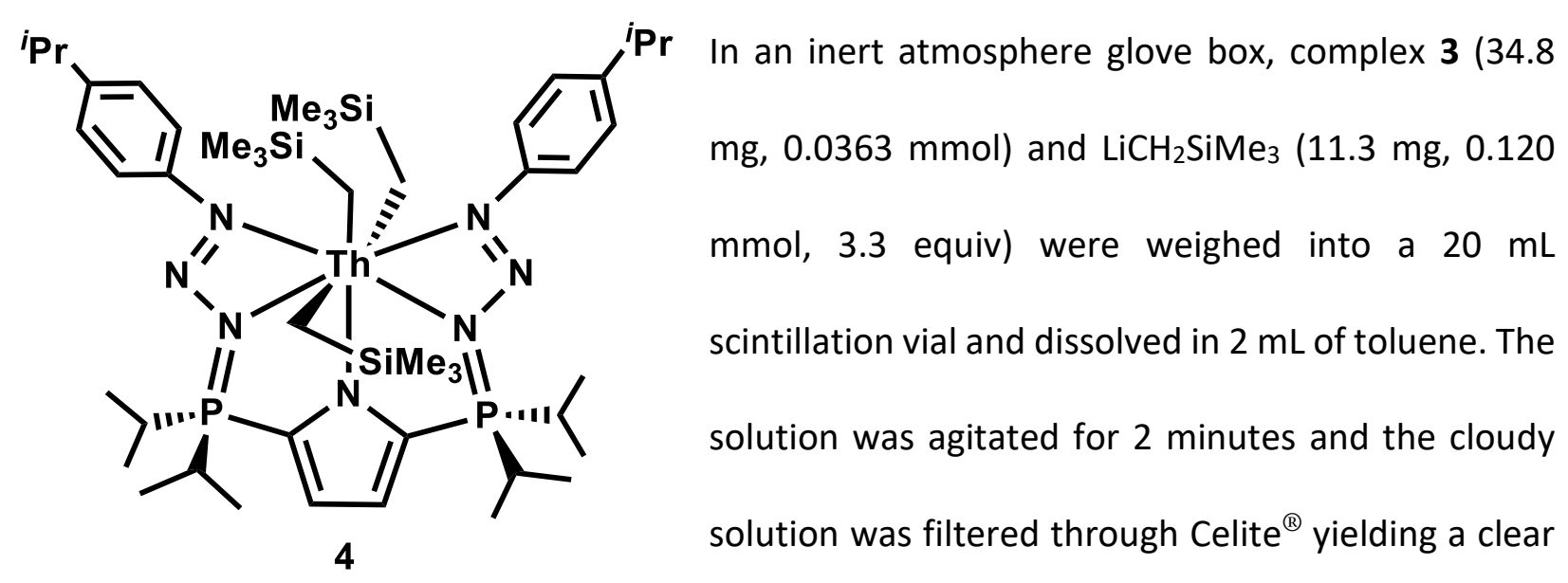

dark orange solution. The toluene was removed in vacuo, resulting in an orange oil. Pentane (2 $\mathrm{mL}$ ) was added to the oil, and the solution was cooled to $-35^{\circ} \mathrm{C}$ for 15 minutes resulting in the formation of X-ray quality crystals. Yield: $30.2 \mathrm{mg}, 71.9 \% .{ }^{1} \mathrm{H} N \mathrm{NMR}\left(\mathrm{C}_{6} \mathrm{D}_{6}, 300.13 \mathrm{MHz}\right): \delta 0.15(\mathrm{br}$ $\left.\mathrm{s}, 6 \mathrm{H}, \mathrm{Th}-\mathrm{CH}_{2}\right), 0.34\left(\mathrm{~s}, 27 \mathrm{H}, \mathrm{Si}\left(\mathrm{CH}_{3}\right)_{3}\right), 1.01\left(\mathrm{~m}, 24 \mathrm{H}, \mathrm{P}-\mathrm{CH}\left(\mathrm{CH}_{3}\right)_{2}\right), 1.12\left(\mathrm{~d},{ }^{3} \mathrm{HHH}_{\mathrm{H}}=6.9 \mathrm{~Hz}, 12 \mathrm{H}, \mathrm{Ar}-\right.$ $\left.\mathrm{CH}\left(\mathrm{CH}_{3}\right)_{2}\right), 2.31\left(\mathrm{dsp},{ }^{3} \mathrm{~J}_{\mathrm{HH}}=7.0 \mathrm{~Hz},{ }^{2} J_{\mathrm{HP}}=3.6 \mathrm{~Hz}, 4 \mathrm{H}, \mathrm{P}-\mathrm{CH}\left(\mathrm{CH}_{3}\right)_{2}\right), 2.71\left(\mathrm{sp},{ }^{3} \mathrm{JHH}_{\mathrm{HH}}=6.9 \mathrm{~Hz}, 2 \mathrm{H}, \mathrm{Ar}-\right.$ $\left.\mathrm{CH}\left(\mathrm{CH}_{3}\right)_{2}\right), 2.44\left(\mathrm{~d},{ }^{3} \mathrm{~J}_{\mathrm{HP}}=6.6 \mathrm{~Hz}, 2 \mathrm{H}\right.$, pyrrole- $\left.\mathrm{CH}\right), 7.30\left(\mathrm{~d}, 3^{3} \mathrm{JH}_{\mathrm{HH}}=8.4 \mathrm{~Hz}, 4 \mathrm{H}\right.$, meta-aromatic $\left.\mathrm{CH}\right)$, $8.13\left(\mathrm{~d},{ }^{3} \mathrm{~J}_{\mathrm{HH}}=8.4 \mathrm{~Hz}, 4 \mathrm{H}\right.$, ortho-aromatic $\left.\mathrm{CH}\right) .{ }^{13} \mathrm{C}\left\{{ }^{1} \mathrm{H}\right\} \mathrm{NMR}\left(\mathrm{C}_{6} \mathrm{D}_{6}, 75.47 \mathrm{MHz}\right): \delta 5.6\left(\mathrm{~s}, \mathrm{Si}\left(\mathrm{CH}_{3}\right)_{3}\right)$, 16.7 (s, P- $\left.\mathrm{CH}\left(\mathrm{CH}_{3}\right)_{2}\right), 16.8$ (s, P- $\left.\mathrm{CH}\left(\mathrm{CH}_{3}\right)_{2}\right), 24.4$ (s, $\left.\mathrm{Ar}-\mathrm{CH}\left(\mathrm{CH}_{3}\right)_{2}\right), 27.0$ (d, ${ }^{1} \mathrm{~J}_{\mathrm{CP}}=49.9 \mathrm{~Hz}, \mathrm{P}-$ $\left.\mathrm{CH}\left(\mathrm{CH}_{3}\right)_{2}\right), 34.51\left(\mathrm{~s}, \mathrm{Ar}-\mathrm{CH}\left(\mathrm{CH}_{3}\right)_{2}\right), 85.4\left(\mathrm{br} \mathrm{s}, \mathrm{Th}-\mathrm{CH}_{2}\right), 119.2\left(\mathrm{dd},{ }^{2} \mathrm{~J}_{\mathrm{CP}}=25.9 \mathrm{~Hz},{ }^{3} \mathrm{~J}_{\mathrm{CP}}=10.4 \mathrm{~Hz}, 3,4-\right.$ pyrrole $\mathrm{CH}$ ), 122.60 (s, aromatic $\mathrm{CH}$ ), $126.0\left(\mathrm{dd},{ }^{1} \mathrm{~J}_{\mathrm{CP}}=133.3 \mathrm{~Hz},{ }^{3} \mathrm{~J}_{\mathrm{CP}}=16.3 \mathrm{~Hz}, 2,5\right.$-pyrrole $\left.C\right), 127.6$ (s, aromatic $\mathrm{CH}$ ), 148.6 (s, aromatic ipso-C), 149.3 (s, aromatic ipso- $\mathrm{C}) .{ }^{31} \mathrm{P}\left\{{ }^{1} \mathrm{H}\right\} \mathrm{NMR}\left(\mathrm{C}_{6} \mathrm{D}_{6}, 121.49\right.$ $\mathrm{MHz}$ ): $\delta 59.4$ (s). Anal. Calcd. (\%) for $\mathrm{C}_{46} \mathrm{H}_{85} \mathrm{~N}_{7} \mathrm{P}_{2} \mathrm{Si}_{3} \mathrm{Th} \bullet$ LiCl: C:47.76; H: 7.41; N: 8.48. Found: C: 47.74; H: 7.46; N: 8.48. The presence of $\mathrm{LiCl}$ was confirmed by ${ }^{7} \mathrm{Li}\left\{{ }^{1} \mathrm{H}\right\} N M R:{ }^{7} \mathrm{Li}\left\{{ }^{1} \mathrm{H}\right\} N M R\left(\mathrm{D}_{2} \mathrm{O}\right.$, 272.22 MHz): $\delta 0.1(\mathrm{~s})$. 


\section{Synthesis of $\mathrm{L}_{\mathrm{P}=\mathrm{N}} \mathrm{ThCl}_{3}$, (5).}

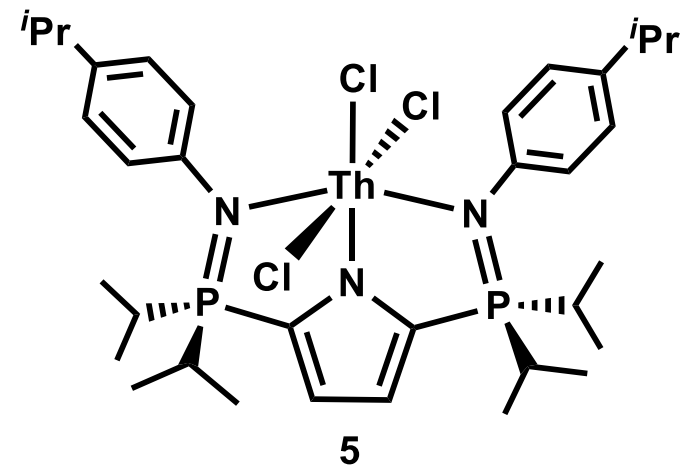

In an inert atmosphere glove box, NaLP=N $(111.2 \mathrm{mg}$, $0.1892 \mathrm{mmol})^{3}$ and $\mathrm{ThCl}_{4}(\mathrm{dme})_{2}(104.8 \mathrm{mg}, 0.1891$ $\mathrm{mmol}$ ) were added to a $20 \mathrm{~mL}$ scintillation vial with a Teflon ${ }^{\circledR}$ coated stir bar. To this mixture, $5 \mathrm{~mL}$ of dme was added and the solution was stirred for one hour.

The solution was filtered through Celite ${ }^{\circledR}$ to remove salts, and the solvent was removed in vacuo. The product was washed with pentane $(2 \times 2 \mathrm{~mL})$ and dried in vacuo resulting in an off-white solid. Yield: $151.7 \mathrm{mg}, 89.4 \% .{ }^{1} \mathrm{H}\left\{{ }^{31} \mathrm{P}\right\} \mathrm{NMR}\left(\mathrm{C}_{6} \mathrm{D}_{6}, 300.13 \mathrm{MHz}\right): \delta 1.01\left(\mathrm{~m}, 24 \mathrm{H}, \mathrm{P}-\mathrm{CH}\left(\mathrm{CH}_{3}\right)_{2}\right), 1.09$ $\left(\mathrm{d},{ }^{3} \mathrm{~J}_{\mathrm{HH}}=7.0 \mathrm{~Hz}, 12 \mathrm{H}, \mathrm{Ar}-\mathrm{CH}\left(\mathrm{CH}_{3}\right)_{2}\right), 1.97\left(\mathrm{~m}, 4 \mathrm{H}, \mathrm{P}-\mathrm{CH}\left(\mathrm{CH}_{3}\right)_{2}\right), 2.64\left(\mathrm{sp},{ }^{3} \mathrm{JHH}_{\mathrm{HH}}=7.0 \mathrm{~Hz}, 2 \mathrm{H}, \mathrm{Ar}-\right.$ $\left.\mathrm{CH}\left(\mathrm{CH}_{3}\right)_{2}\right), 6.54(\mathrm{~s}, 2 \mathrm{H}$, pyrrole- $\mathrm{CH}), 7.11\left(\mathrm{~d},{ }^{3} \mathrm{~J}_{\mathrm{HH}}=8.4 \mathrm{~Hz}, 4 \mathrm{H}\right.$, meta-aromatic $\left.\mathrm{CH}\right), 7.75\left(\mathrm{~d},{ }^{3} \mathrm{JHH}_{\mathrm{HH}}=\right.$ $8.4 \mathrm{~Hz}, 4 \mathrm{H}$, orth-aromatic $\mathrm{CH}) .{ }^{13} \mathrm{C}\left\{{ }^{1} \mathrm{H}\right\} \operatorname{NMR}\left(\mathrm{C}_{6} \mathrm{D}_{6}, 75.47 \mathrm{MHz}\right): \delta 16.4\left(\mathrm{~d},{ }^{2} J_{\mathrm{CP}}=45.4 \mathrm{~Hz}, \mathrm{P}-\right.$ $\left.\mathrm{CH}\left(\mathrm{CH}_{3}\right)_{2}\right), 24.4\left(\mathrm{~s}, \mathrm{Ar}-\mathrm{CH}\left(\mathrm{CH}_{3}\right)_{2}\right), 27.7\left(\mathrm{~d},{ }^{1}{ }_{\mathrm{CP}}=53.4 \mathrm{~Hz}, \mathrm{P}-\mathrm{CH}\left(\mathrm{CH}_{3}\right)_{2}\right), 34.2\left(\mathrm{~s}, \mathrm{Ar}-\mathrm{CH}\left(\mathrm{CH}_{3}\right)_{2}\right), 116.6$ $\left(\mathrm{dd},{ }^{2} J_{\mathrm{CP}}=25.8 \mathrm{~Hz},{ }^{3} J_{\mathrm{CP}}=9.9 \mathrm{~Hz}, 3,4\right.$-pyrrole $\left.C \mathrm{H}\right), 129.7$ (s, aromatic $\left.C \mathrm{H}\right), 130.0\left(\mathrm{~d},{ }^{3} J_{\mathrm{CP}}=5.2 \mathrm{~Hz}\right.$ aromatic $\mathrm{CH}$ ), 135.6 (s, aromatic ipso- $C$ ), 148.2 (s, aromatic ipso- $C$ ). The 2,5-pyrrole $C$ resonance overlapped with the benzene- $d_{6}$ solvent, but was observed using $d_{8}$-THF: $130.2\left(\mathrm{dd},{ }^{1} J_{\mathrm{CP}}=132.1\right.$ $\mathrm{Hz},{ }^{3} J_{\mathrm{CP}}=14.3 \mathrm{~Hz}, 2,5$-pyrrole $\left.C\right) .{ }^{31} \mathrm{P}\left\{{ }^{1} \mathrm{H}\right\} \operatorname{NMR}\left(\mathrm{C}_{6} \mathrm{D}_{6}, 121.49 \mathrm{MHz}\right): \delta 56.1(\mathrm{~s})$. Anal. Calcd. (\%) for $\mathrm{C}_{34} \mathrm{H}_{52} \mathrm{~N}_{3} \mathrm{P}_{2}$ Th: C: 45.22; H: 5.80; N: 4.65. Found: C: 45.16; H: 5.89; N: 4.42. 


\section{Synthesis of $\mathrm{LP}_{\mathrm{P}=\mathrm{N}} * \mathrm{Th}\left(\mathrm{CH}_{2} \mathrm{SiMe}_{3}\right)_{2} \bullet 2 \mathrm{LiCl},(6 \bullet 2 \mathrm{LiCl})$.}

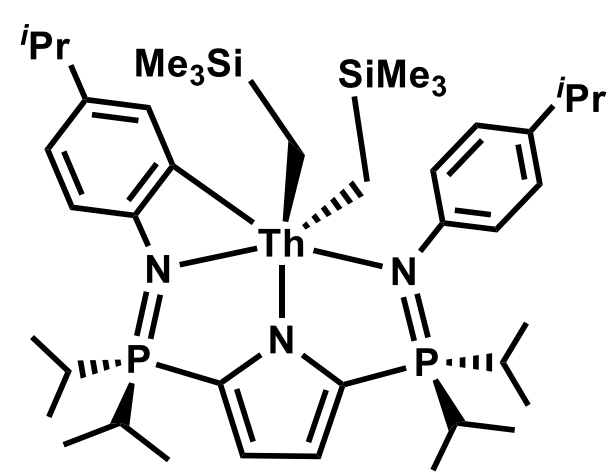

6

In an inert atmosphere glove box, complex 5 (29.8 mg, $0.0330 \mathrm{mmol})$ and $\mathrm{LiCH}_{2} \mathrm{SiMe}_{3}(9.3 \mathrm{mg}, 0.099 \mathrm{mmol}, 3$ equiv) were weighed into a $20 \mathrm{~mL}$ scintillation vial and dissolved in toluene $(2 \mathrm{~mL})$. The vial was agitated for 2 minutes and the cloudy solution was quickly filtered through Celite ${ }^{\circledR}$ to remove salts. The toluene was removed in vacuo yielding an off-white sticky solid. The product was washed with pentane $(3 \times 1 \mathrm{~mL})$ cooled to $-35^{\circ} \mathrm{C}$ and dried in vacuo to yield a crystalline white solid. X-ray quality crystals were grown from pentane at $-35{ }^{\circ} \mathrm{C}$ in 15 minutes. Yield: $18.8 \mathrm{mg}, 54.0 \% .{ }^{1} \mathrm{H}\left\{{ }^{31} \mathrm{P}\right\}$ NMR $\left(\mathrm{C}_{6} \mathrm{D}_{6}, 700.44\right.$ $\mathrm{MHz}): \delta 0.02\left(\mathrm{~d},{ }^{2}{ }_{\mathrm{HH}}=11.5 \mathrm{~Hz}, 2 \mathrm{H}, \mathrm{Th}-\mathrm{CH}_{2}\right), 0.29\left(\mathrm{~d},{ }^{2}{ }_{\mathrm{HH}}=11.5 \mathrm{~Hz}, 2 \mathrm{H}, \mathrm{Th}-\mathrm{CH}_{2}\right), 0.33(\mathrm{~s}, 18 \mathrm{H}$, $\left.\mathrm{Si}\left(\mathrm{CH}_{3}\right)_{3}\right), 0.90$ (ov m, 24H, P-CH(CH3) $\left.)_{2}\right), 1.22\left(\mathrm{~d},{ }^{3} \mathrm{~J}_{\mathrm{HH}}=6.9 \mathrm{~Hz}, 6 \mathrm{H}, \mathrm{Ar}-\mathrm{CH}\left(\mathrm{CH}_{3}\right)_{2}\right), 1.44\left(\mathrm{~d},{ }^{3} \mathrm{~J}_{\mathrm{HH}}=6.9\right.$ $\left.\mathrm{Hz}, 6 \mathrm{H}, \mathrm{Ar}-\mathrm{CH}\left(\mathrm{CH}_{3}\right)_{2}\right), 1.99\left(\mathrm{sp},{ }^{3} \mathrm{~J}_{\mathrm{HH}}=7.1 \mathrm{~Hz}, 2 \mathrm{H}, \mathrm{P}-\mathrm{CH}\left(\mathrm{CH}_{3}\right)_{2}\right), 2.20\left(\mathrm{sp}, 3^{3} \mathrm{HH}_{\mathrm{H}}=7.1 \mathrm{~Hz}, 2 \mathrm{H}, \mathrm{P}-\right.$ $\left.\mathrm{CH}\left(\mathrm{CH}_{3}\right)_{2}\right), 2.79\left(\mathrm{sp}, 3^{3} \mathrm{HH}_{1}=6.9 \mathrm{~Hz}, 1 \mathrm{H}, \mathrm{Ar}-\mathrm{CH}\left(\mathrm{CH}_{3}\right)_{2}\right), 3.00\left(\mathrm{sp},{ }^{3} J_{\mathrm{HH}}=6.9 \mathrm{~Hz}, 1 \mathrm{H}, \mathrm{Ar}-\mathrm{CH}\left(\mathrm{CH}_{3}\right)_{2}\right), 6.42$ $\left(d,{ }^{3} J_{H H}=3.3 \mathrm{~Hz}, 1 \mathrm{H}\right.$, pyrrole-CH), $6.49\left(\mathrm{~d},{ }^{3} \mathrm{~J}_{\mathrm{HH}}=3.3 \mathrm{~Hz}, 1 \mathrm{H}\right.$, pyrrole- $\left.\mathrm{CH}\right), 6.54\left(\mathrm{~d},{ }^{3} \mathrm{~J}_{\mathrm{HH}}=7.8 \mathrm{~Hz}, 1 \mathrm{H}\right.$, ortho-cyclometallated $\mathrm{Ar}-\mathrm{CH}$ ), $7.13\left(\mathrm{dd},{ }^{3} \mathrm{~J}_{\mathrm{HH}}=7.8 \mathrm{~Hz},{ }^{4} \mathrm{~J}_{\mathrm{HH}}=2.0 \mathrm{~Hz}, 1 \mathrm{H}\right.$, meta-cyclometallated $\operatorname{Ar}-\mathrm{CH}), 7.28\left(\mathrm{~d},{ }^{3} \mathrm{~J}_{\mathrm{HH}}=8.2 \mathrm{~Hz}, 2 \mathrm{H}\right.$, meta-aromatic $\left.\mathrm{CH}\right), 7.46\left(\mathrm{~d}, 3^{3} \mathrm{JH}_{\mathrm{HH}}=8.2 \mathrm{~Hz}, 2 \mathrm{H}\right.$, ortho-aromatic $\mathrm{CH}), 8.30\left(\mathrm{~d},{ }^{4} \mathrm{~J}_{\mathrm{HH}}=2.1 \mathrm{~Hz}, 1 \mathrm{H}\right.$, meta-cyclometallated $\left.\mathrm{Ar}-\mathrm{CH}\right) .{ }^{13} \mathrm{C}\left\{{ }^{1} \mathrm{H}\right\} \mathrm{NMR}\left(\mathrm{C}_{6} \mathrm{D}_{6}, 176.13 \mathrm{MHz}\right): \delta$ $4.4\left(\mathrm{~s}, \mathrm{Si}\left(\mathrm{CH}_{3}\right)_{3}\right), 15.9\left(\mathrm{~d},{ }^{2} J_{\mathrm{CP}}=2.3 \mathrm{~Hz} \mathrm{P}-\mathrm{CH}\left(\mathrm{CH}_{3}\right)_{2}\right), 16.3\left(\mathrm{~d},{ }^{2} J_{\mathrm{CP}}=1.8 \mathrm{~Hz}, \mathrm{P}-\mathrm{CH}\left(\mathrm{CH}_{3}\right)_{2}\right), 16.4\left(\mathrm{~d},{ }^{2} J_{\mathrm{CP}}\right.$ $\left.=2.0 \mathrm{~Hz}, \mathrm{P}-\mathrm{CH}\left(\mathrm{CH}_{3}\right)_{2}\right), 16.8\left(\mathrm{~d},{ }^{2} \mathrm{~J}_{\mathrm{CP}}=2.3 \mathrm{~Hz}, \mathrm{P}-\mathrm{CH}\left(\mathrm{CH}_{3}\right)_{2}\right), 24.7\left(\mathrm{~s}, \mathrm{Ar}-\mathrm{CH}\left(\mathrm{CH}_{3}\right)_{2}\right), 25.5$ (s, $\mathrm{Ar}-$ $\left.\mathrm{CH}\left(\mathrm{CH}_{3}\right)_{2}\right), 26.8\left(\mathrm{~d},{ }^{1} J_{\mathrm{CP}}=53.3 \mathrm{~Hz}, \mathrm{P}-\mathrm{CH}\left(\mathrm{CH}_{3}\right)_{2}\right), 26.9\left(\mathrm{~d},{ }^{1} \mathrm{~J}_{\mathrm{CP}}=53.3 \mathrm{~Hz}, \mathrm{P}-\mathrm{CH}\left(\mathrm{CH}_{3}\right)_{2}\right), 34.2(\mathrm{~s}, \mathrm{Ar}-$ $\left.\mathrm{CH}\left(\mathrm{CH}_{3}\right)_{2}\right), 35.3\left(\mathrm{~s}, \mathrm{Ar}-\mathrm{CH}\left(\mathrm{CH}_{3}\right)_{2}\right), 87.3\left(\mathrm{~s}, \mathrm{Th}-\mathrm{CH}_{2}\right), 116.9$ (s, aromatic $\left.\mathrm{CH}\right), 117.4\left(\mathrm{dd},{ }^{2} \mathrm{~J}_{\mathrm{CP}}=25.6\right.$ 
$\mathrm{Hz},{ }^{3} J_{\mathrm{CP}}=10.4 \mathrm{~Hz}, 3,4$-pyrrole $\mathrm{CH}$ ), $119.2\left(\mathrm{dd},{ }^{2} \mathrm{~J}_{\mathrm{CP}}=24.4 \mathrm{~Hz},{ }^{3} \mathrm{JP}_{\mathrm{CP}}=10.4 \mathrm{~Hz}, 3,4\right.$-pyrrole $\mathrm{CH}$ ), 125.0 (s, aromatic $\mathrm{CH}), 128.7(\mathrm{~s}$, aromatic $\mathrm{CH}), 128.8\left(\mathrm{~d},{ }^{3} \mathrm{CP}_{\mathrm{CP}}=6.4 \mathrm{~Hz}\right.$, aromatic $\left.\mathrm{CH}\right), 130.3\left(\mathrm{dd},{ }^{1} J_{\mathrm{CP}}=\right.$ 136.4 Hz, ${ }^{3} J_{\mathrm{CP}}=14.4 \mathrm{~Hz}, 2,5$-pyrrole $C$ ), $132.3\left(\mathrm{dd},{ }^{1} \mathrm{JP}_{\mathrm{CP}}=139.4 \mathrm{~Hz},{ }^{3} \mathrm{JP}_{\mathrm{CP}}=13.5 \mathrm{~Hz}, 2,5\right.$-pyrrole $C$ ), $135.8\left(\mathrm{~d},{ }^{4} \mathrm{~J}_{\mathrm{CP}}=5.0 \mathrm{~Hz}\right.$, aromatic $\left.\mathrm{CH}\right), 140.8\left(\mathrm{~s}\right.$, aromatic ipso-C), $143.8\left(\mathrm{~d},{ }^{2} \mathrm{~J}_{\mathrm{CP}}=4.7 \mathrm{~Hz}\right.$, aromatic ipso-C), $145.0\left(\mathrm{~d},{ }^{5} \mathrm{JP}_{\mathrm{CP}}=3.6 \mathrm{~Hz}\right.$, aromatic ipso-C), $150.8\left(\mathrm{~d},{ }^{2} \mathrm{~J}_{\mathrm{CP}}=3.7 \mathrm{~Hz}\right.$, aromatic ipso-C), $203.5(\mathrm{br}$ s, cyclometallated Th-C). ${ }^{31} \mathrm{P}\left\{{ }^{1} \mathrm{H}\right\}$ NMR $\left(\mathrm{C}_{6} \mathrm{D}_{6}, 283.54 \mathrm{MHz}\right): \delta 47.7$ (s), 49.5 (s). Anal. Calcd. (\%) for $\mathrm{C}_{42} \mathrm{H}_{73} \mathrm{~N}_{3} \mathrm{P}_{2} \mathrm{Si}_{2} \mathrm{Th} \cdot 2 \mathrm{LiCl}: \mathrm{C}: 47.82 ; \mathrm{H}: 6.97 ; \mathrm{N}: 3.98$. Found: C: $47.81 ; \mathrm{H}: 6.97 ; \mathrm{N}: 4.00$. The presence of LiCl was confirmed by ${ }^{7} \mathrm{Li}\left\{{ }^{1} \mathrm{H}\right\} \mathrm{NMR}:{ }^{7} \mathrm{Li}\left\{{ }^{1} \mathrm{H}\right\} \mathrm{NMR}\left(\mathrm{D}_{2} \mathrm{O}, 272.22 \mathrm{MHz}\right): \delta 0.1(\mathrm{~s})$. 
II. NMR Spectra.

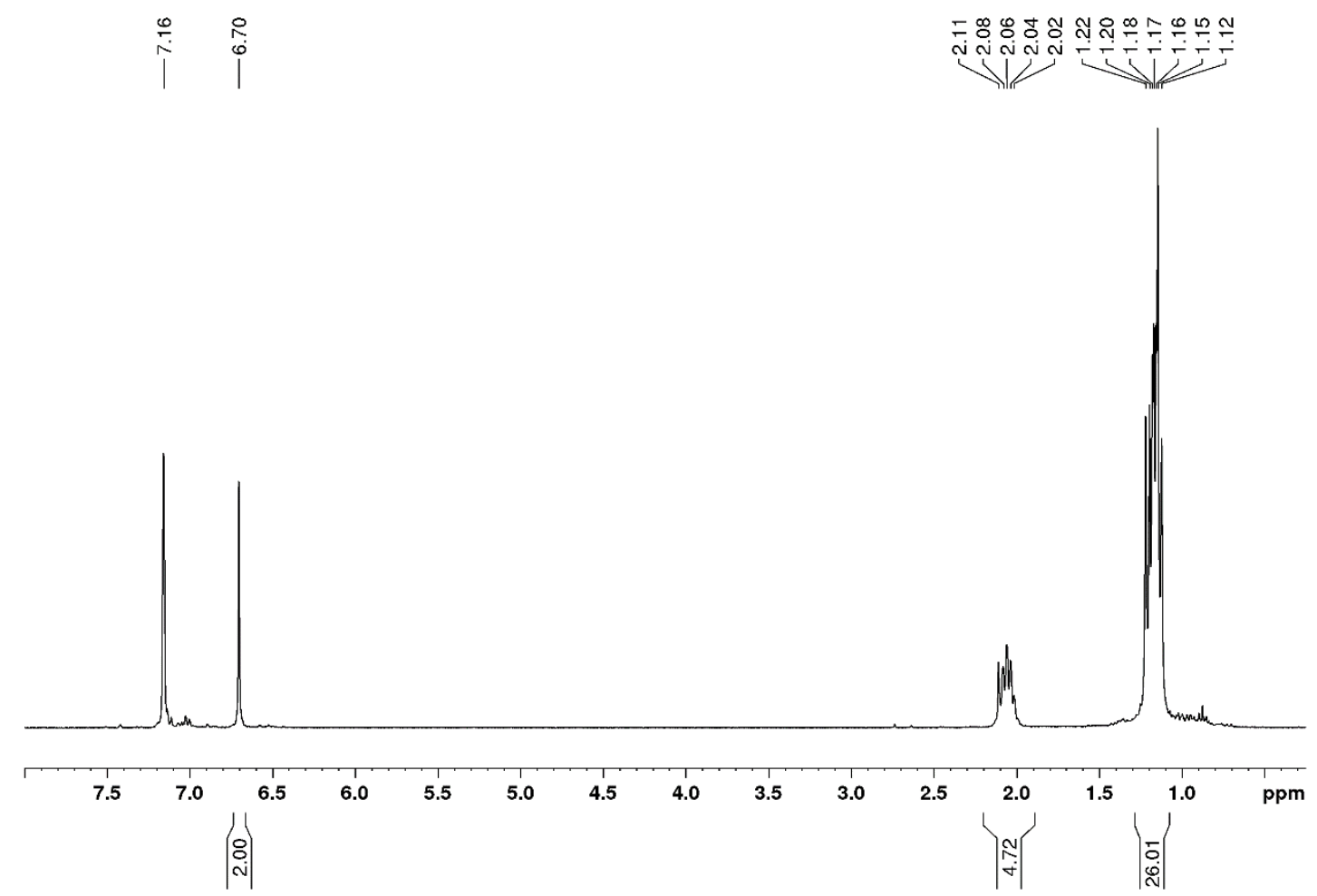

Figure S1: ${ }^{1} \mathrm{H}$ NMR spectrum of 2,5-bis(diisopropylphosphino)-N-K-pyrrole (1) (benzene- $d_{6}$ ).

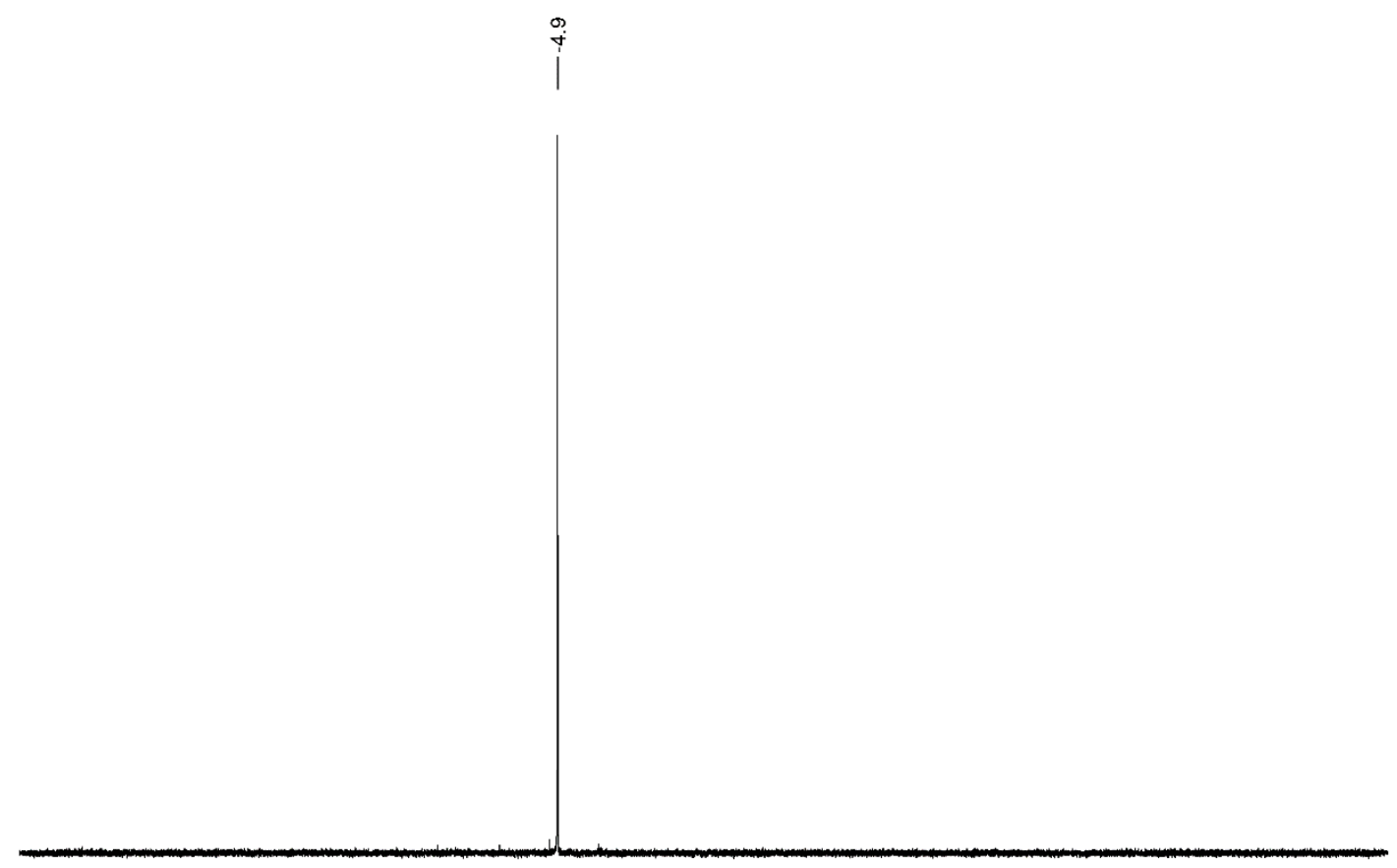

Figure S2: ${ }^{31} \mathrm{P}\left\{{ }^{1} \mathrm{H}\right\}$ NMR spectrum of 2,5-bis(diisopropylphosphino)-N-K-pyrrole (1) (benzene- $d_{6}$ ). 


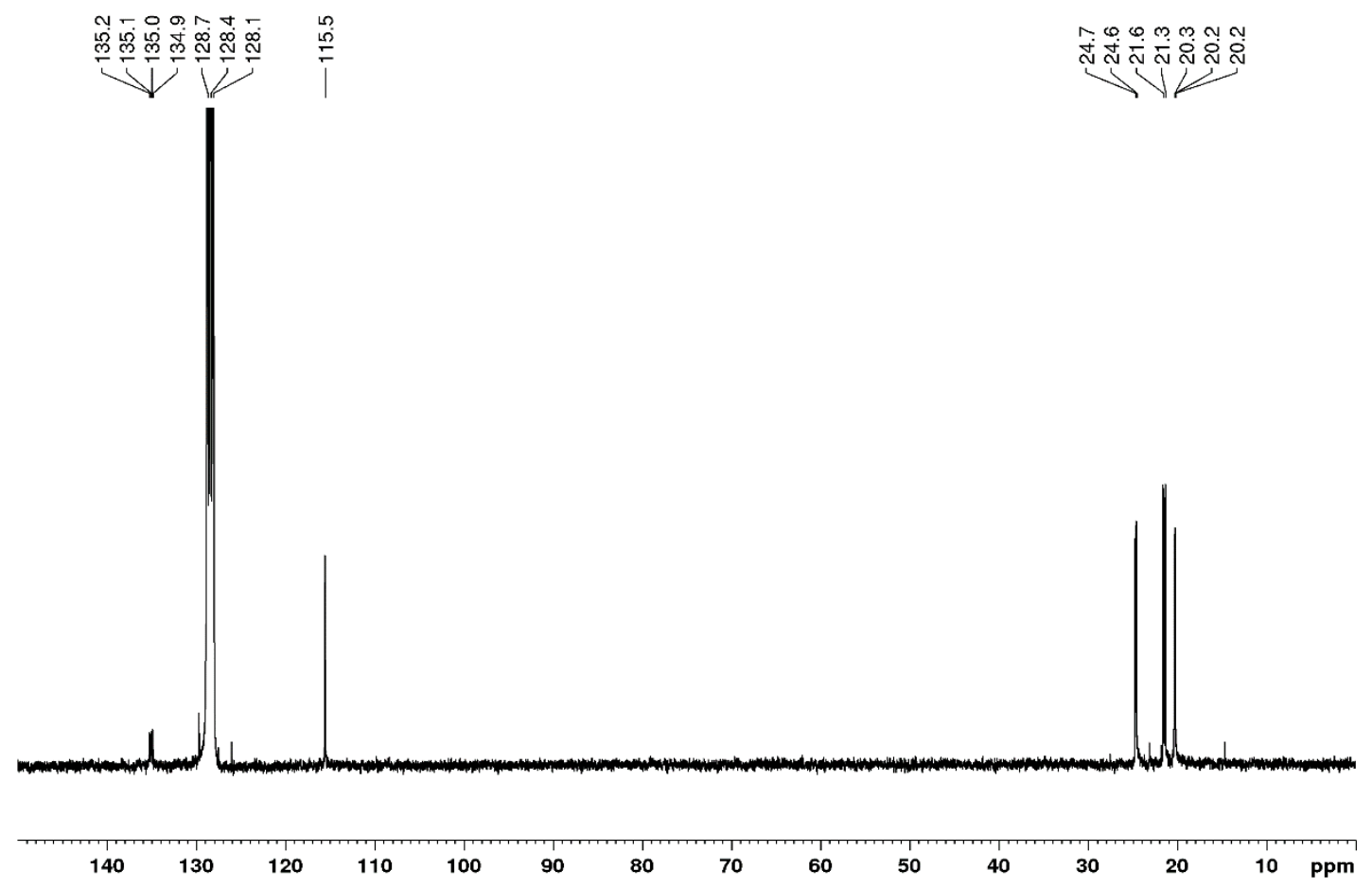

Figure S3: ${ }^{13} \mathrm{C}\left\{{ }^{1} \mathrm{H}\right\}$ NMR spectrum of 2,5-bis(diisopropylphosphino)-N-K-pyrrole (1) (benzene- $d_{6}$ ).

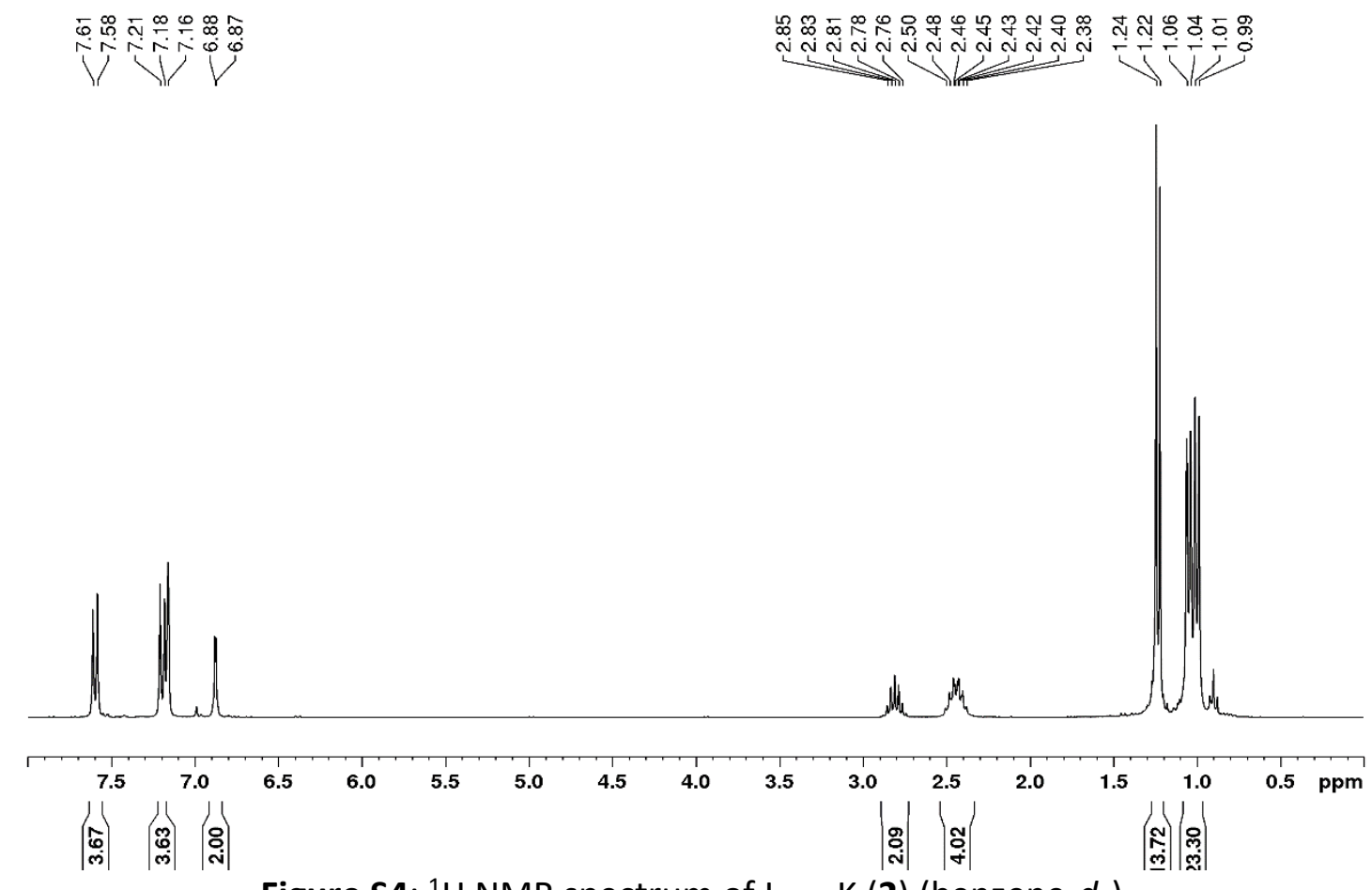

Figure S4: ${ }^{1} \mathrm{H}$ NMR spectrum of $\mathrm{L}_{\mathrm{P}=\mathrm{N} 3} \mathrm{~K}(2)$ (benzene- $d_{6}$ ). 


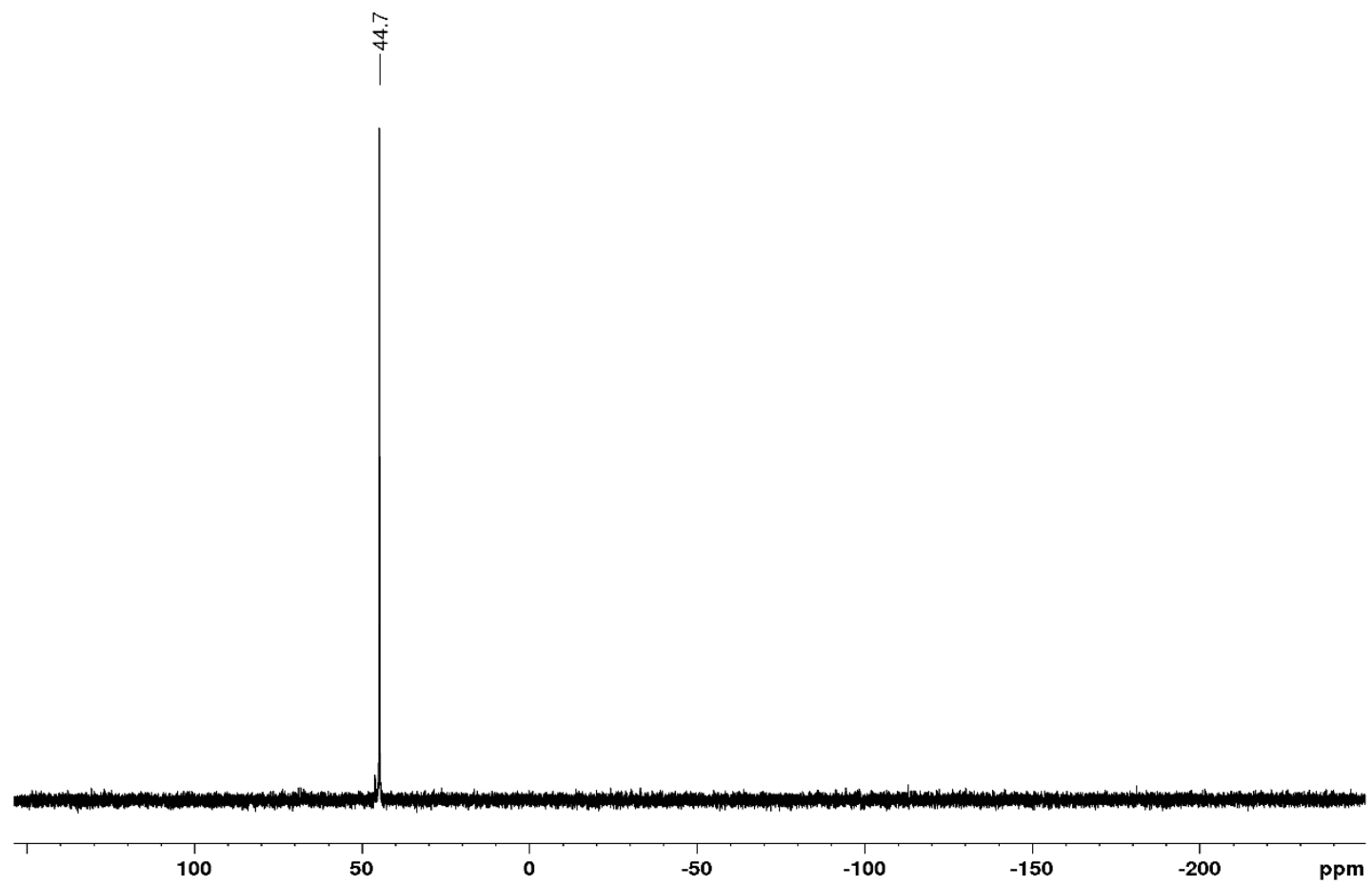

Figure S5: ${ }^{31} \mathrm{P}\left\{{ }^{1} \mathrm{H}\right\}$ NMR spectrum of $\mathrm{L}_{\mathrm{P}=\mathrm{N} 3} \mathrm{~K}(2)$ (benzene- $d_{6}$ ).

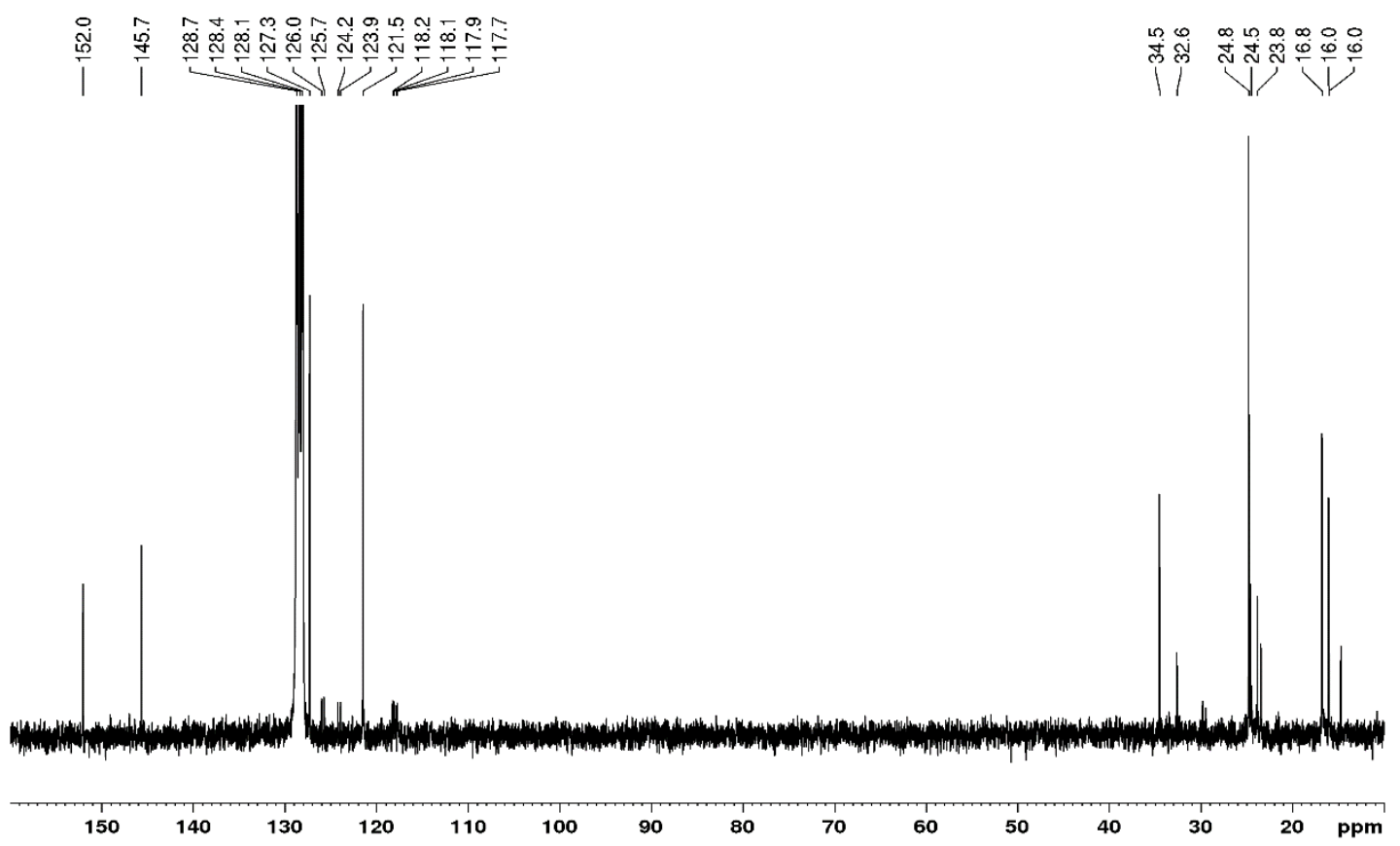

Figure S6: $\left.{ }^{13} \mathrm{C}^{1}{ }^{1} \mathrm{H}\right\}$ NMR spectrum of $\mathrm{LP}_{\mathrm{P}} \mathrm{N}_{3} \mathrm{~K}(2)$ (benzene- $d_{6}$ ). 


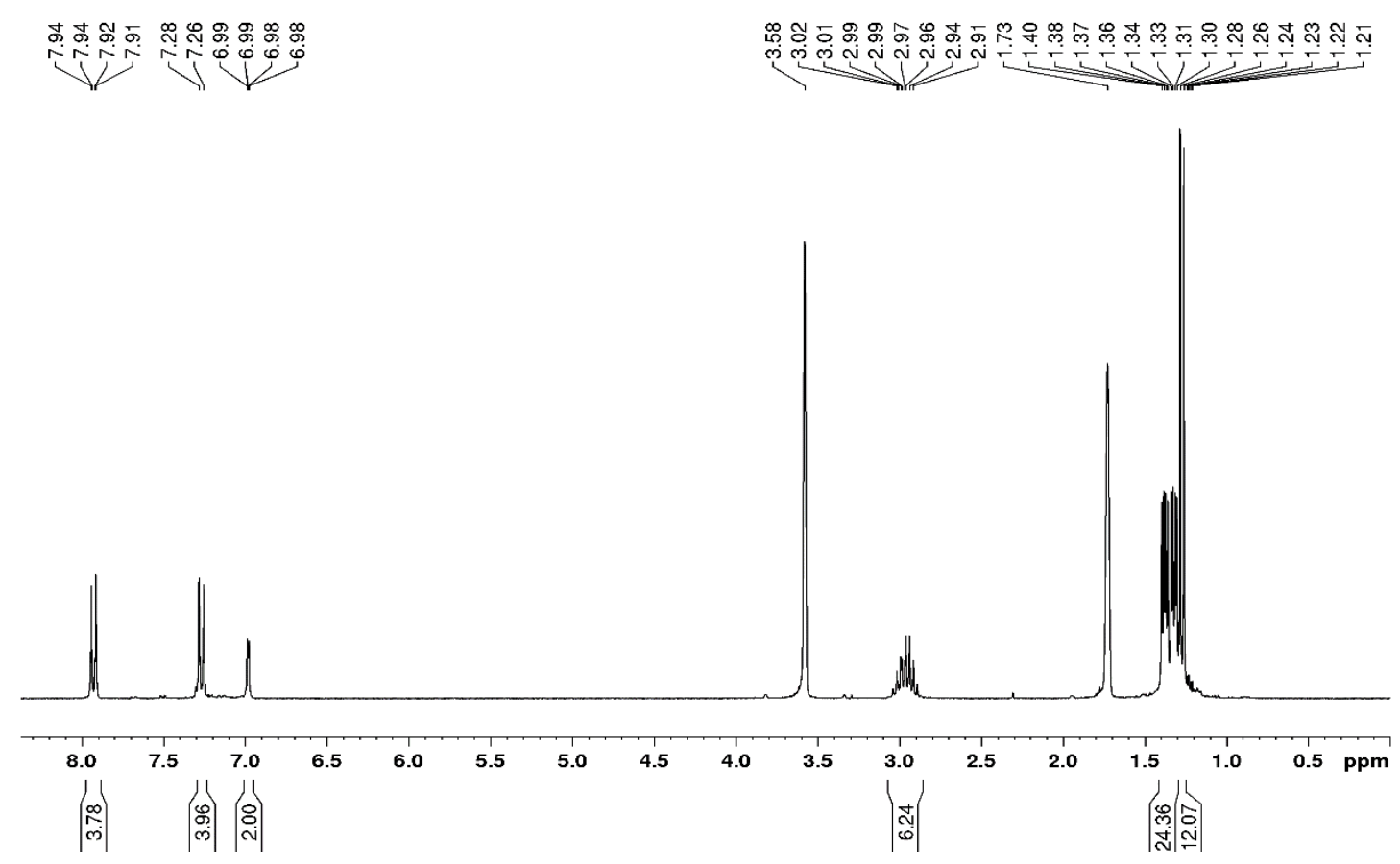

Figure S7: ${ }^{1} \mathrm{H}$ NMR spectrum of $\mathrm{L}_{\mathrm{P}=\mathrm{N} 3} \mathrm{ThCl}_{3}(3)\left(\mathrm{THF}-\mathrm{d}_{8}\right)$.

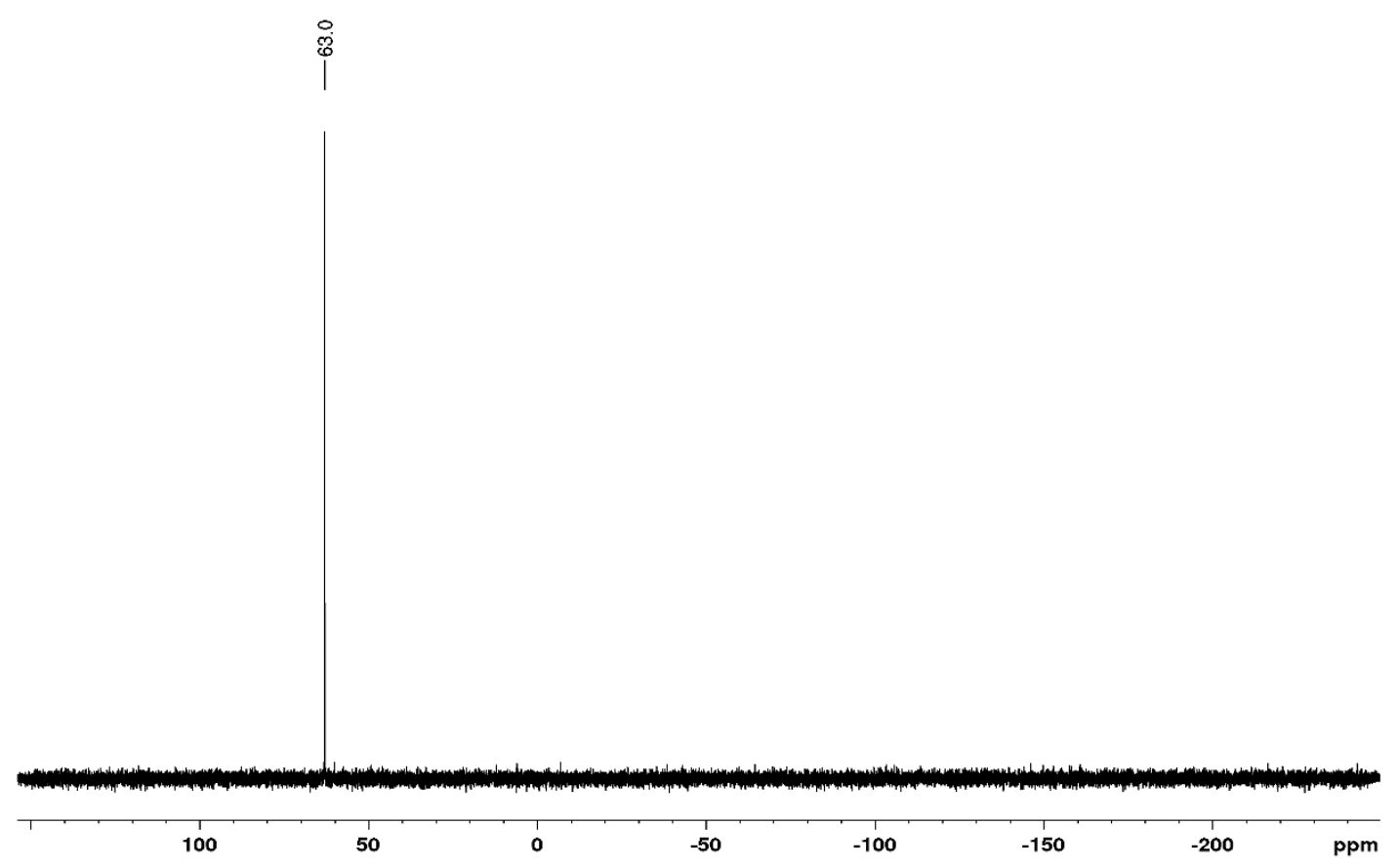

Figure S8: ${ }^{11} \mathrm{P}\left\{{ }^{1} \mathrm{H}\right\}$ NMR spectrum of $\mathrm{L}_{\mathrm{P}=\mathrm{N} 3} \mathrm{ThCl}_{3}(3)$ (benzene- $d_{6}$ ). 

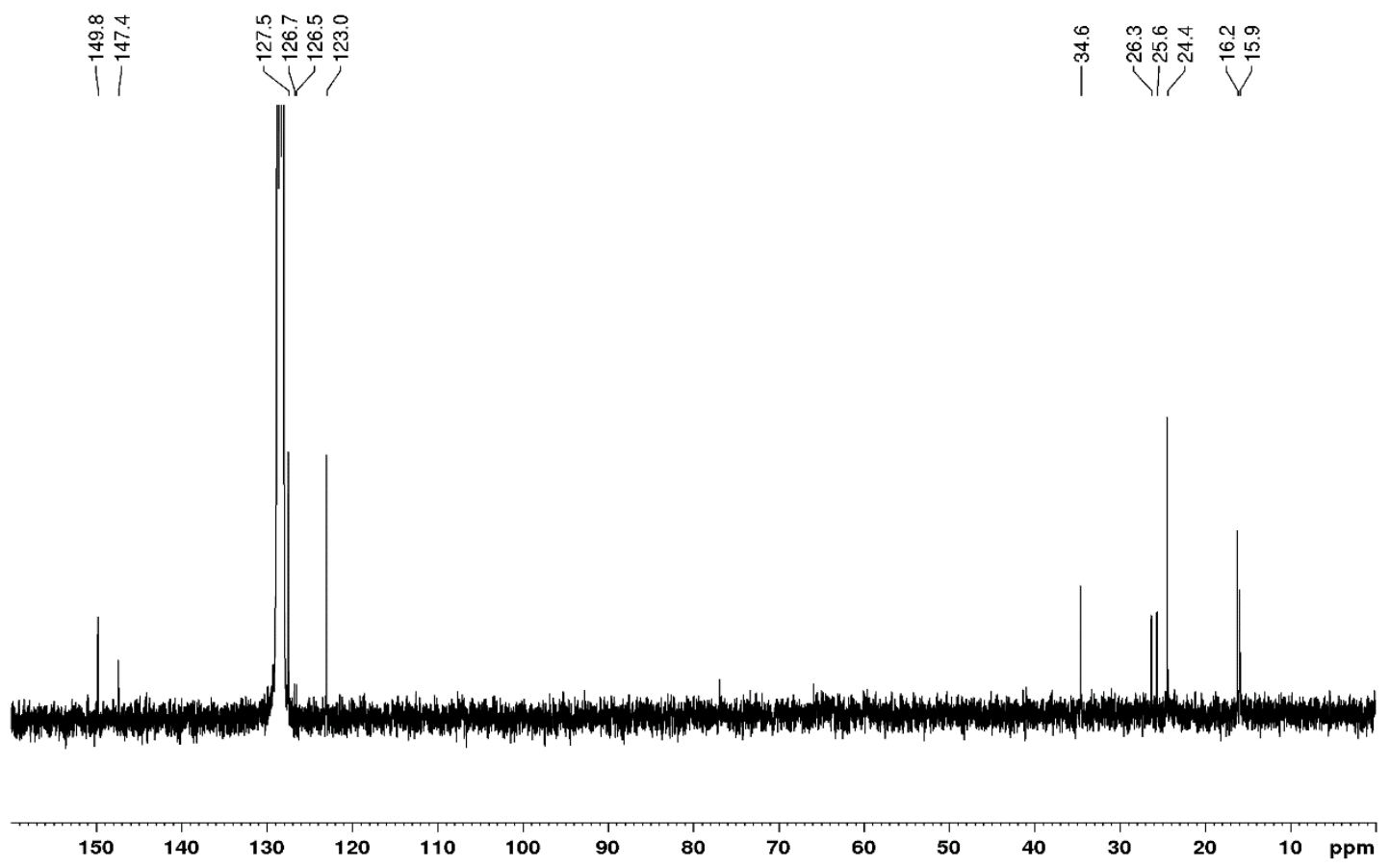

Figure S9: ${ }^{13} \mathrm{C}\left\{{ }^{1} \mathrm{H}\right\}$ NMR spectrum of $\mathrm{LP}_{\mathrm{N}=\mathrm{N} 3} \mathrm{ThCl}_{3}(3)$ (benzene- $d_{6}$ ).

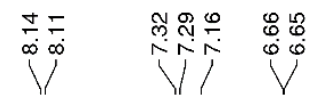

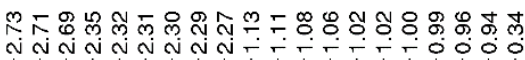
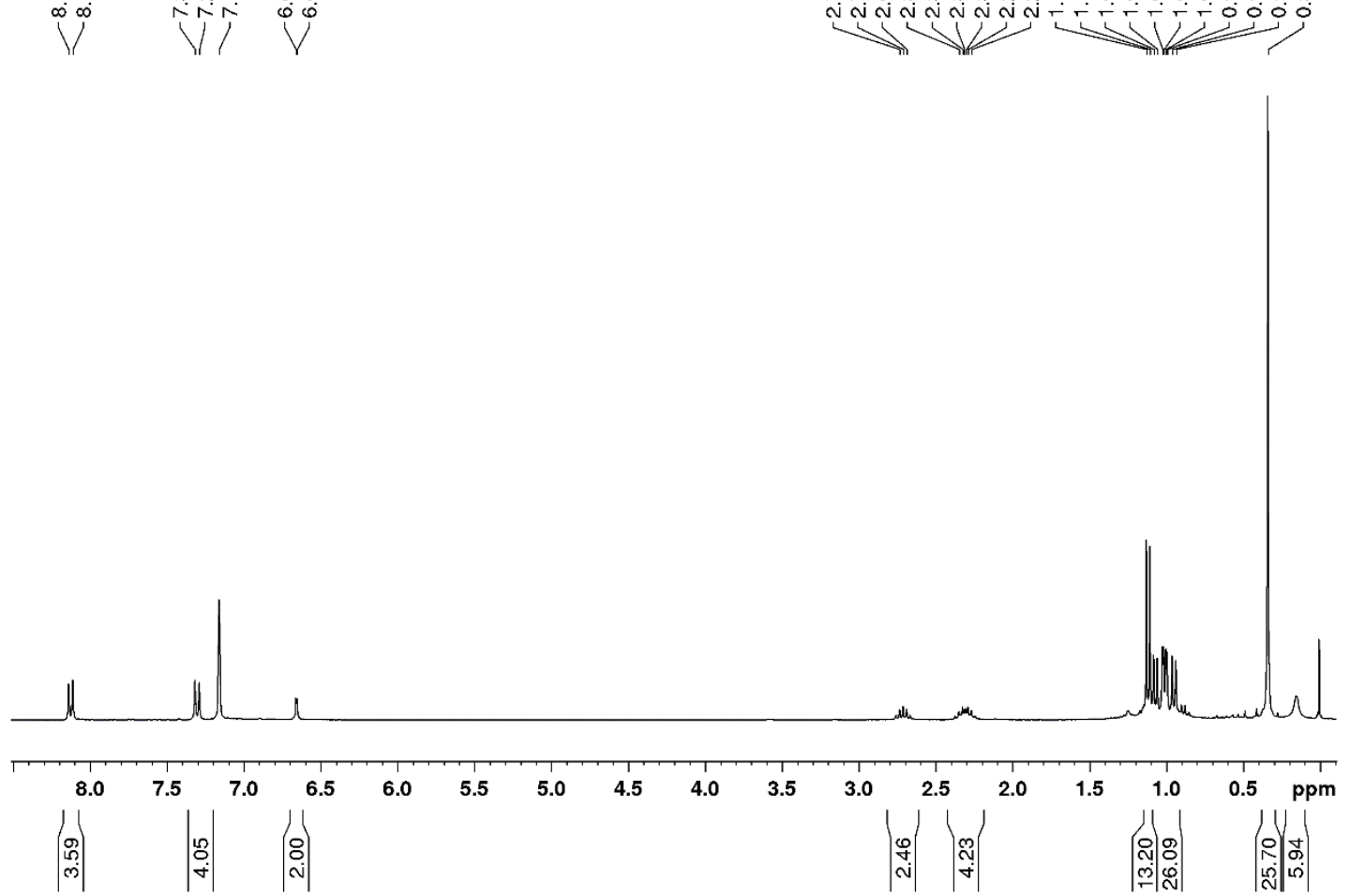

Figure S10: ${ }^{1} \mathrm{H}$ NMR spectrum of $\left.\mathrm{LP}_{\mathrm{p}=\mathrm{N} 3} \mathrm{Th}\left(\mathrm{CH}_{2} \mathrm{SiMe}\right)_{3}\right)_{3} \mathrm{LiCl}(4 \cdot \mathrm{LiCl})\left(\right.$ benzene- $\left.d_{6}\right)$. 


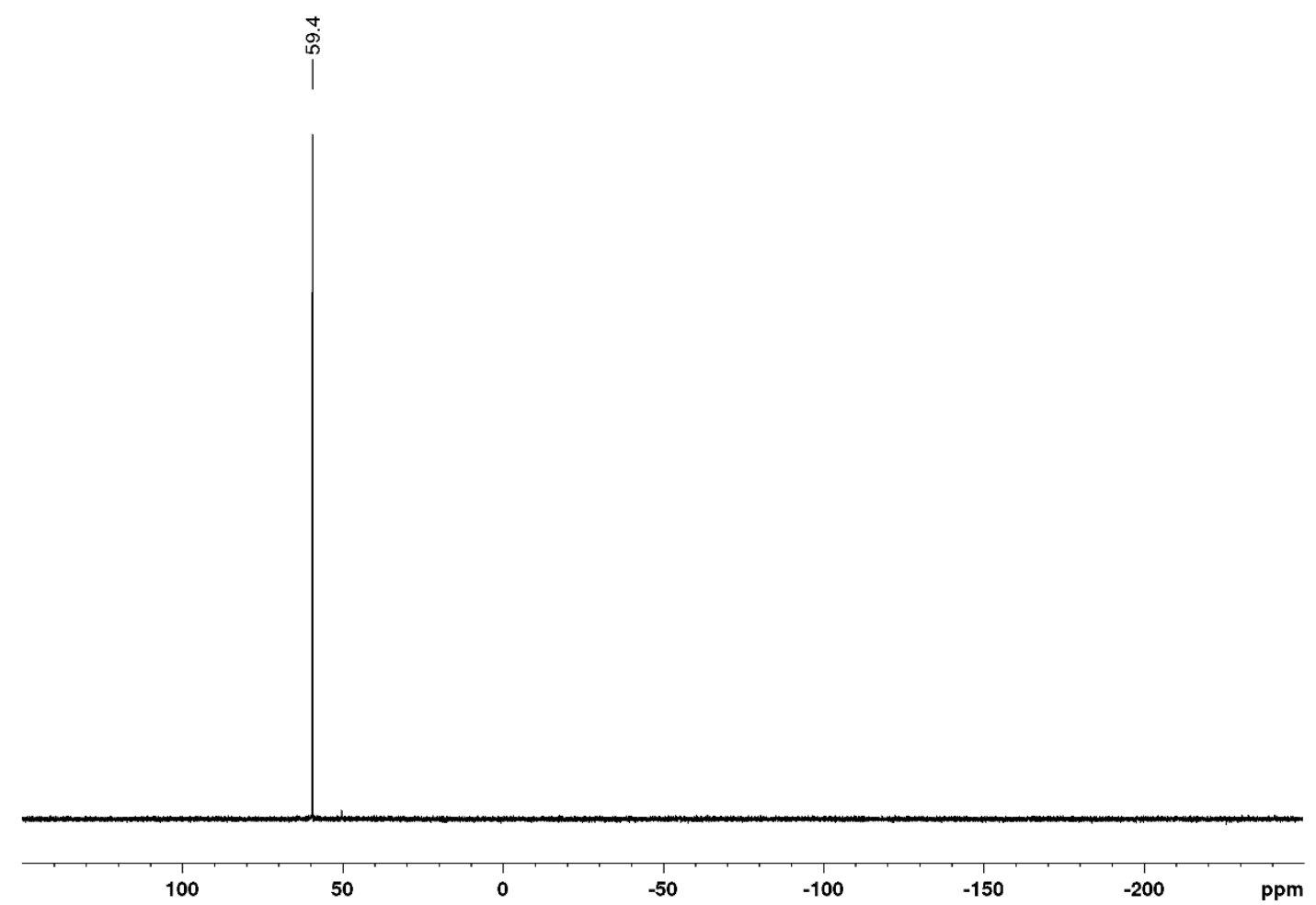

Figure S11: ${ }^{31} \mathrm{P}\left\{{ }^{1} \mathrm{H}\right\}$ NMR spectrum of $\mathrm{LP}_{\mathrm{P}=\mathrm{N} 3} \mathrm{Th}\left(\mathrm{CH}_{2} \mathrm{SiMe}_{3}\right)_{3} \bullet \mathrm{LiCl}(\mathbf{4} \bullet \mathrm{LiCl})$ (benzene- $\left.d_{6}\right)$.

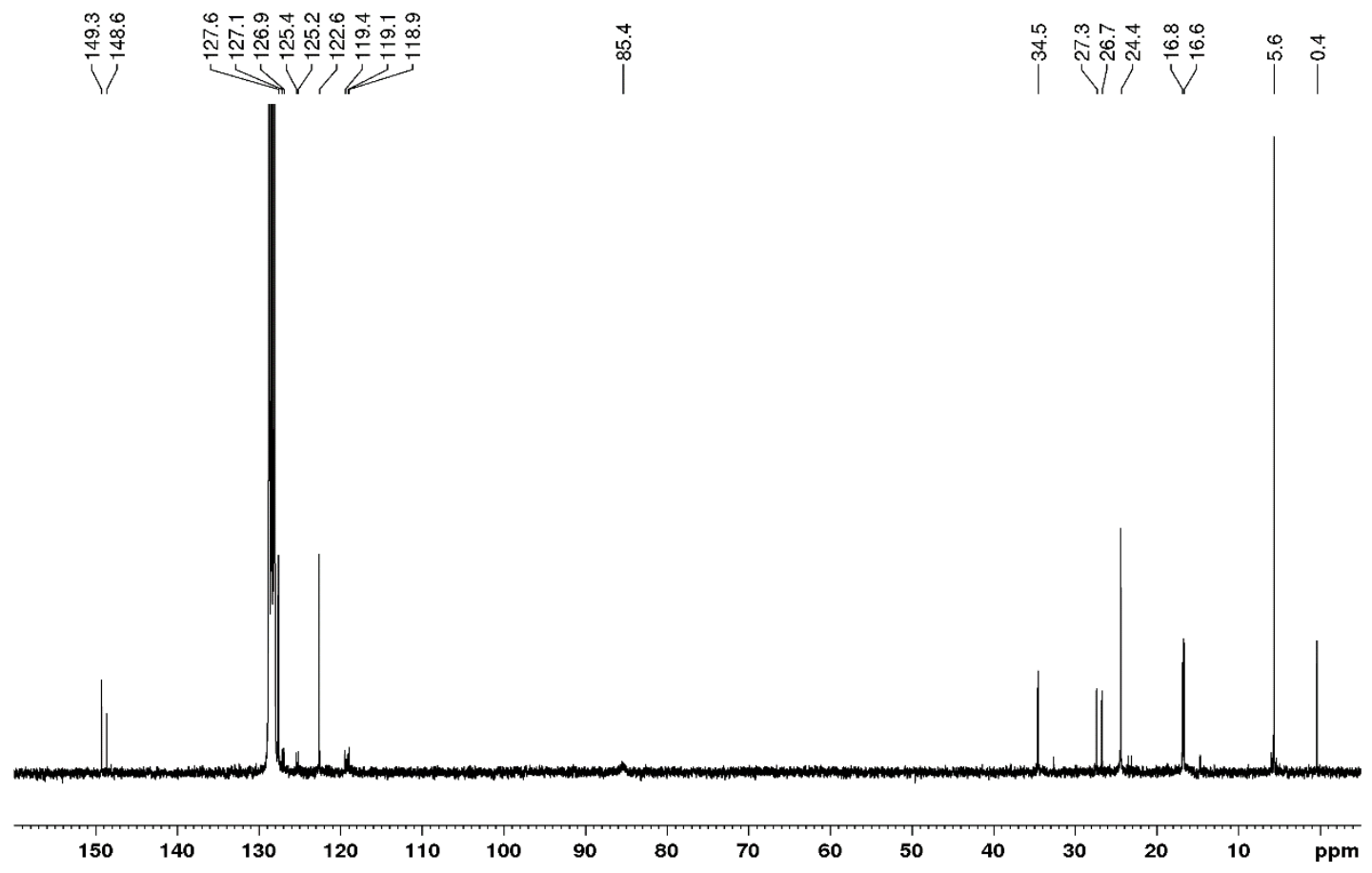

Figure S12: ${ }^{13} \mathrm{C}\left\{{ }^{1} \mathrm{H}\right\}$ NMR spectrum of $\mathrm{LP}_{\mathrm{P}=\mathrm{N} 3} \mathrm{Th}\left(\mathrm{CH}_{2} \mathrm{SiMe}_{3}\right)_{3} \bullet \mathrm{LiCl}(\mathbf{4} \bullet \mathrm{LiCl})$ (benzene- $\left.d_{6}\right)$. 


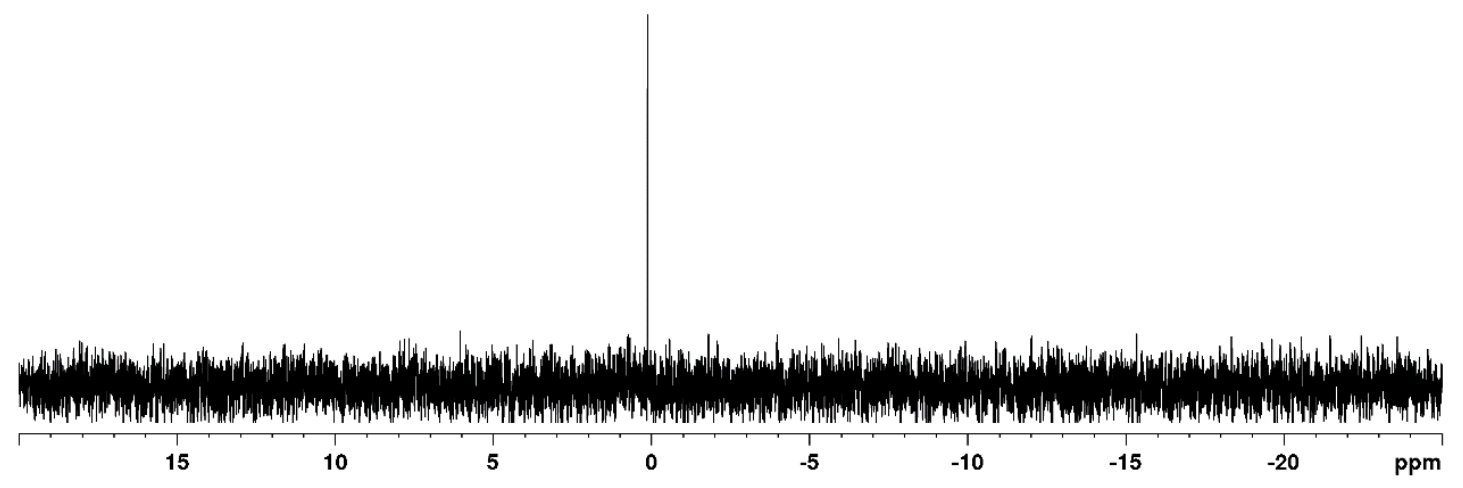

Figure S13: ${ }^{7} \mathrm{Li}\left\{{ }^{1} \mathrm{H}\right\}$ NMR spectrum of $\mathrm{LP}_{\mathrm{P}=\mathrm{N} 3} \mathrm{Th}\left(\mathrm{CH}_{2} \mathrm{SiMe}_{3}\right)_{3} \bullet \mathrm{LiCl}(4 \bullet \mathrm{LiCl})\left(\mathrm{D}_{2} \mathrm{O}\right)$.

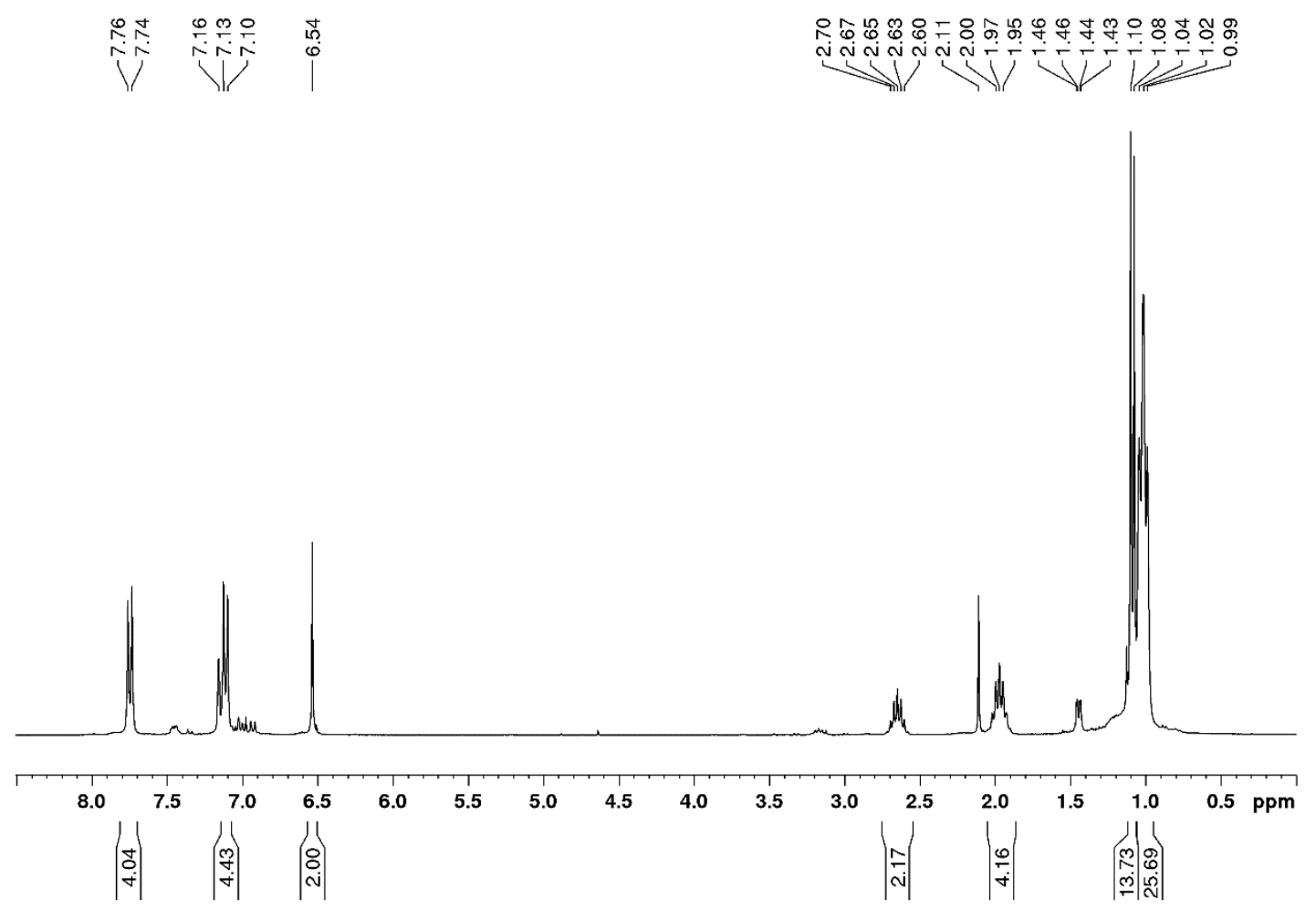

Figure S14: ${ }^{1} \mathrm{H}\left\{{ }^{31} \mathrm{P}\right\} \mathrm{NMR}$ spectrum of $\mathrm{L}_{\mathrm{P}=\mathrm{N}} \mathrm{ThCl}_{3}(5)$ (benzene- $d_{6}$ ). 


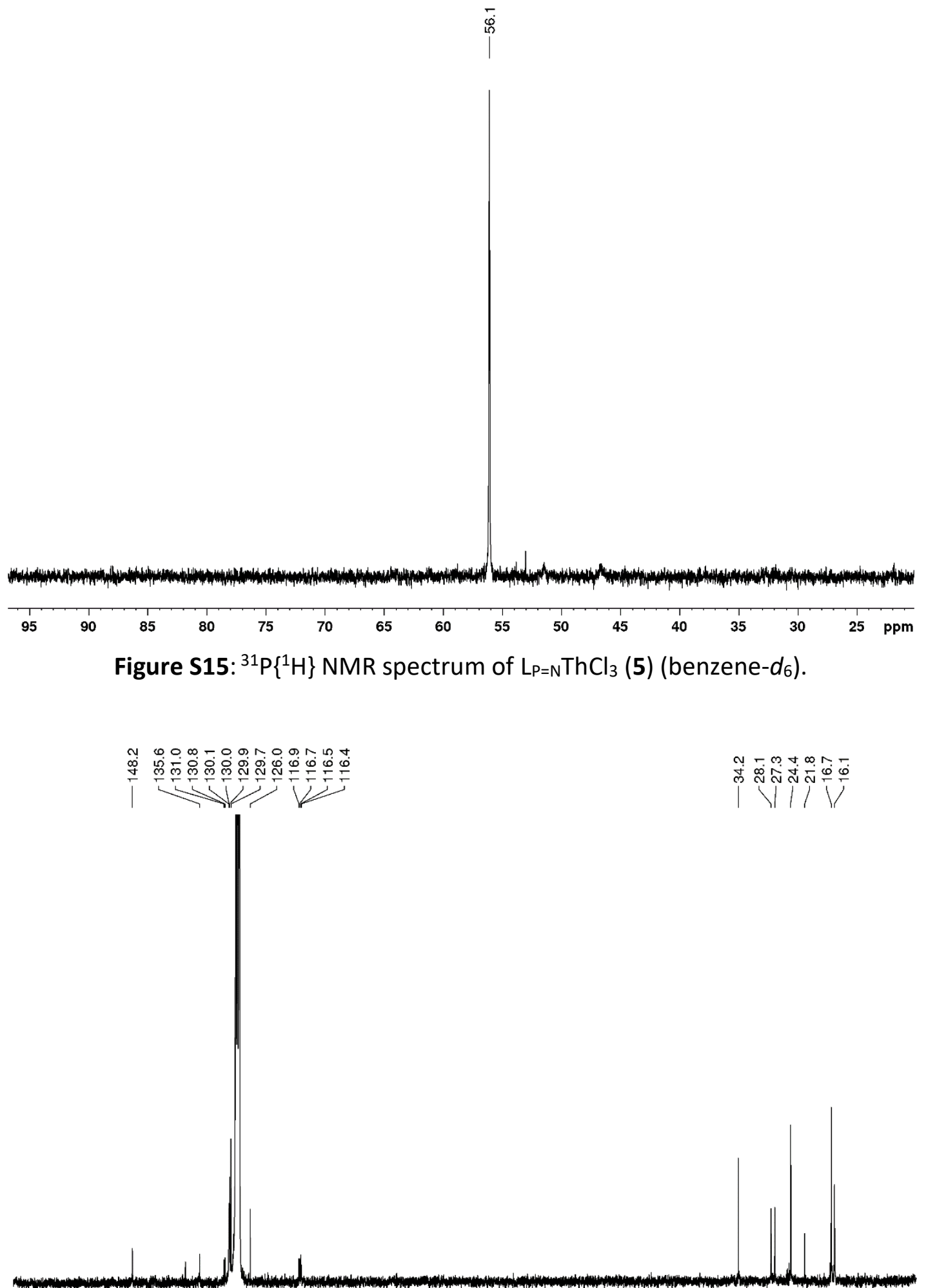

$\begin{array}{lllllllllllllllll}160 & 150 & 140 & 130 & 120 & 110 & 100 & 90 & 80 & 70 & 60 & 50 & 40 & 30 & 20 & 10 & \mathrm{ppm}\end{array}$ Figure S16: ${ }^{13} \mathrm{C}\left\{{ }^{1} \mathrm{H}\right\}$ NMR spectrum of $\mathrm{L}_{\mathrm{P}=\mathrm{N}} \mathrm{ThCl}_{3}(5)$ (benzene- $d_{6}$ ). 


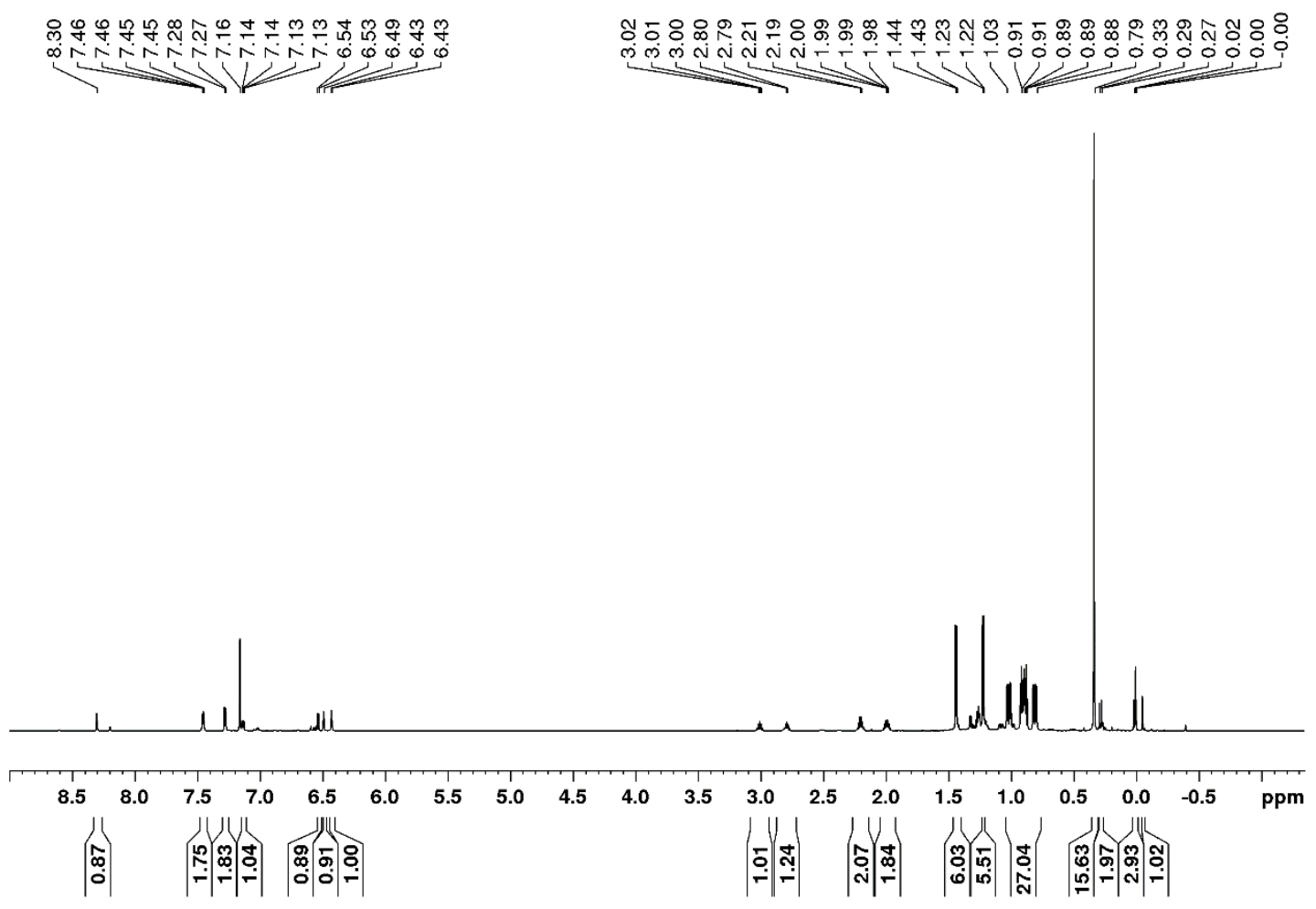

Figure S17: ${ }^{1} \mathrm{H}$ NMR spectrum of $\mathrm{L}_{\mathrm{p}=\mathrm{N}} * \mathrm{Th}\left(\mathrm{CH}_{2} \mathrm{SiMe}_{3}\right)_{2} \bullet 2 \mathrm{LiCl}(\mathbf{6} \bullet \mathbf{2} \mathrm{LiCl})\left(\right.$ benzene- $\left.d_{6}\right)$.
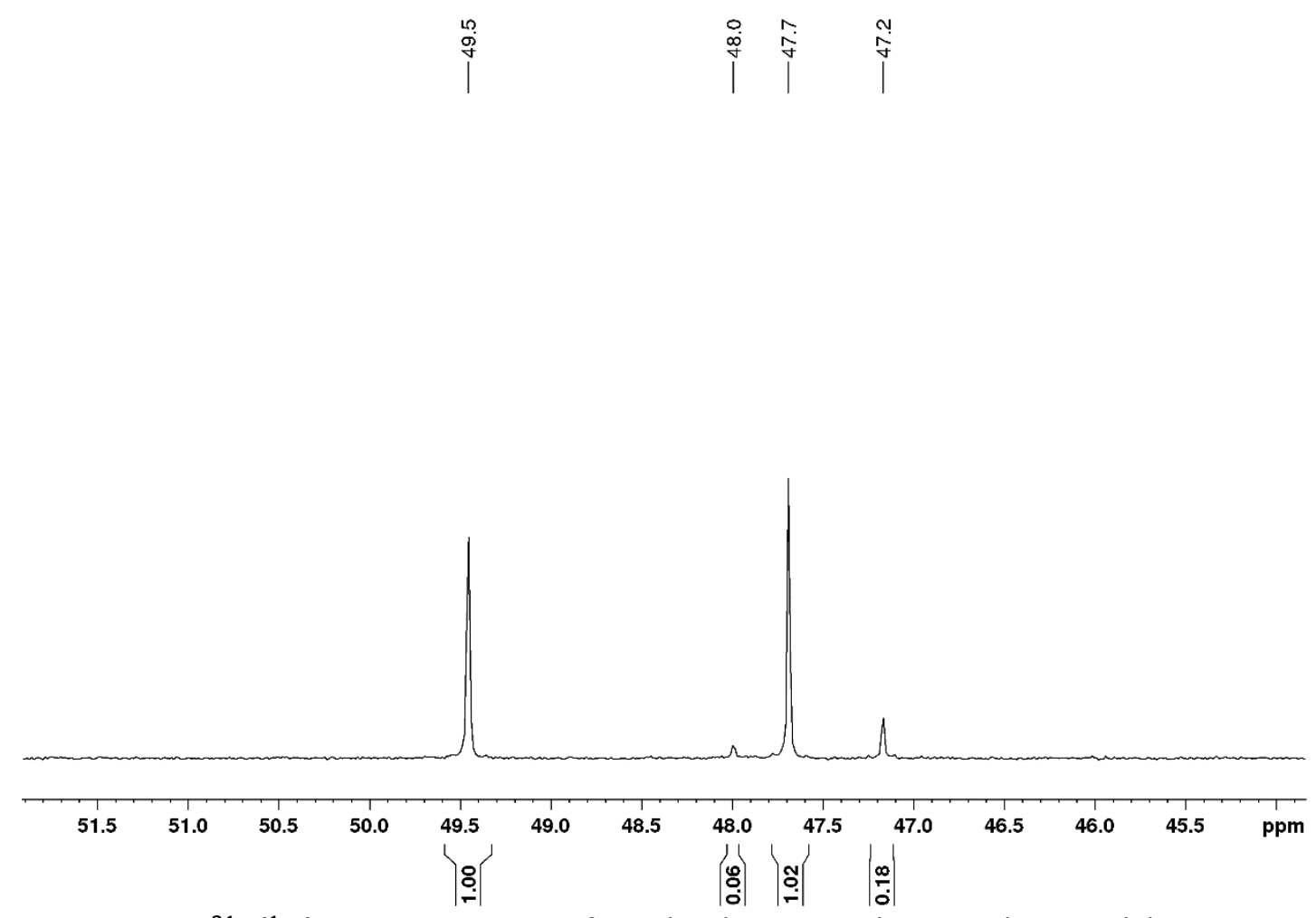

Figure S18: ${ }^{31} \mathrm{P}\left\{{ }^{1} \mathrm{H}\right\}$ NMR spectrum of $\mathrm{LP}_{\mathrm{P}} * \mathrm{Th}\left(\mathrm{CH}_{2} \mathrm{SiMe}_{3}\right)_{2} \bullet 2 \mathrm{LiCl}(\mathbf{6} \bullet \mathbf{2} \mathrm{LiCl})$ (benzene$d_{6}$ ). 


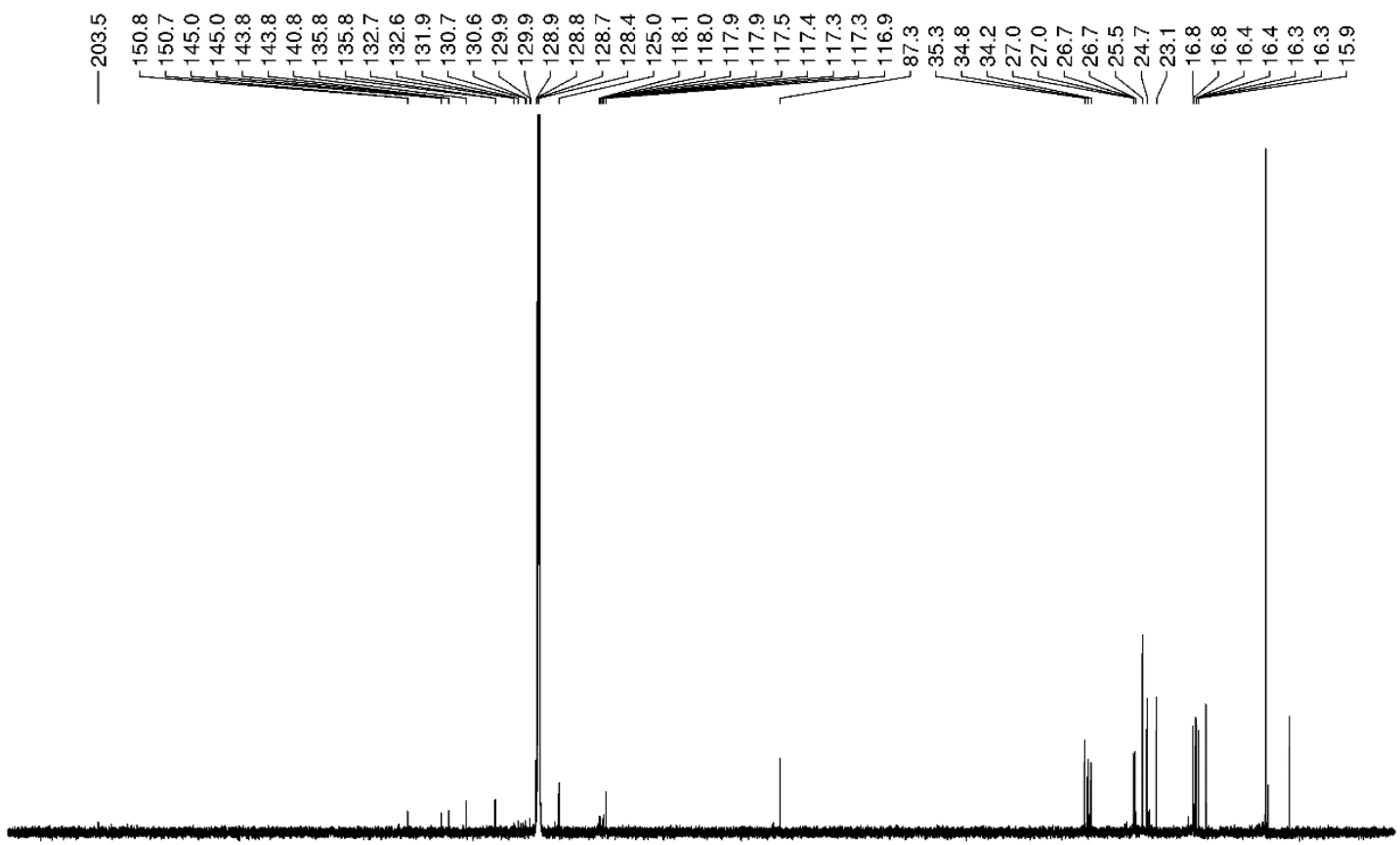

$\begin{array}{llllllllllllllllllllllll}210 & 200 & 190 & 180 & 170 & 160 & 150 & 140 & 130 & 120 & 110 & 100 & 90 & 80 & 70 & 60 & 50 & 40 & 30 & 20 & 10 & 0 & \mathrm{ppm}\end{array}$ Figure S19: ${ }^{13} \mathrm{C}\left\{{ }^{1} \mathrm{H}\right\}$ NMR spectrum of $\mathrm{LP}_{\mathrm{P}=\mathrm{N}} * \mathrm{Th}\left(\mathrm{CH}_{2} \mathrm{SiMe}_{3}\right)_{2} \bullet 2 \mathrm{LiCl}(\mathbf{6} \bullet \mathbf{2} \mathrm{LiCl})$ (benzenedal

Figure S20: ${ }^{7} \mathrm{Li}\left\{{ }^{1} \mathrm{H}\right\} \mathrm{NMR}$ spectrum of $\mathrm{LP}_{\mathrm{N}}{ }^{*} \mathrm{Th}\left(\mathrm{CH}_{2} \mathrm{SiMe}_{3}\right)_{2} \bullet 2 \mathrm{LiCl}(\mathbf{6} \bullet \mathbf{2} \mathrm{LiCl})\left(\mathrm{D}_{2} \mathrm{O}\right)$. 


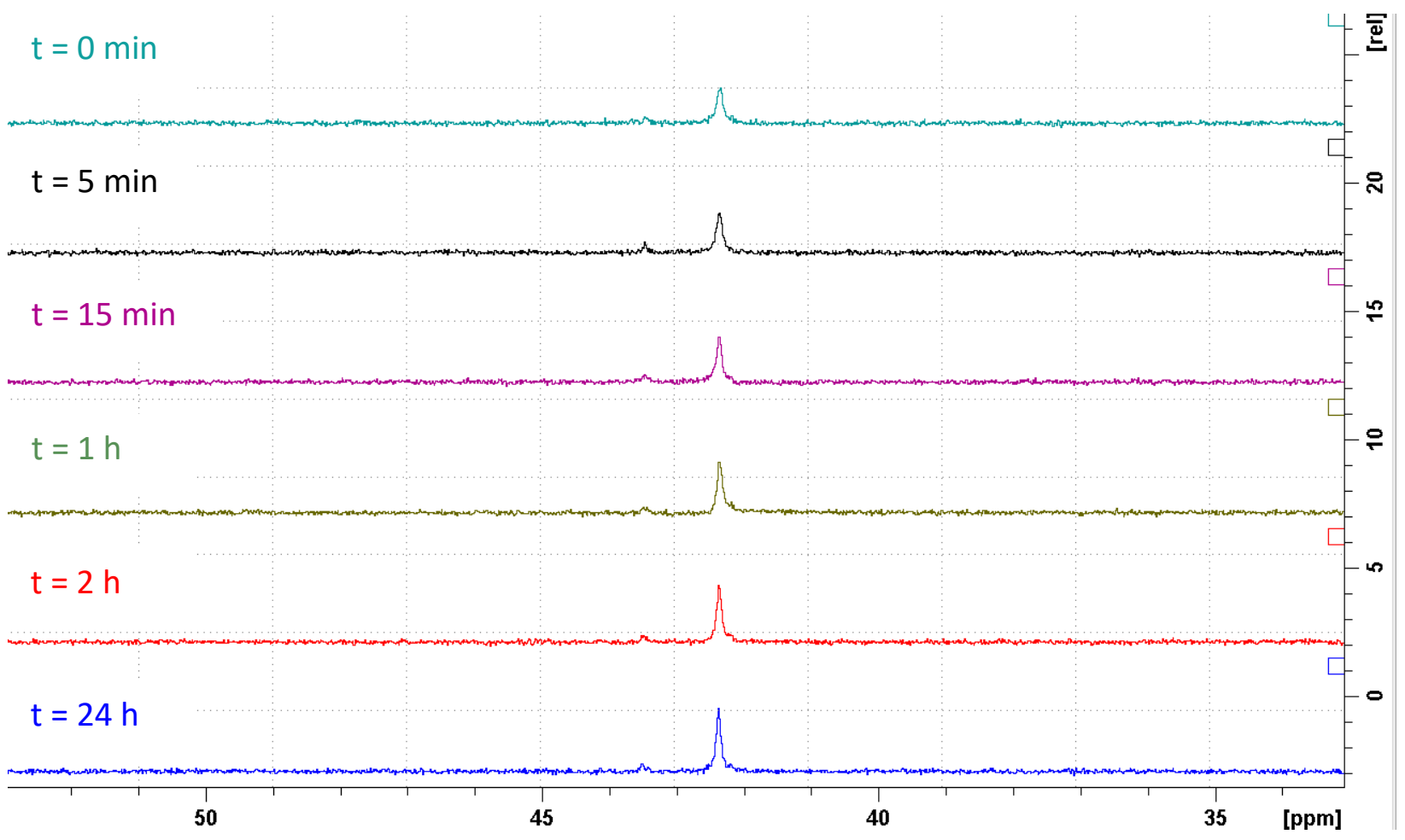

Figure S21: ${ }^{31} \mathrm{P}\left\{{ }^{1} \mathrm{H}\right\}$ NMR spectra of $\mathrm{L}_{\mathrm{P}=\mathrm{N} 3} \mathrm{~K}(2)$ taken at different time intervals at ambient temperature (benzene- $d_{6}$ ).

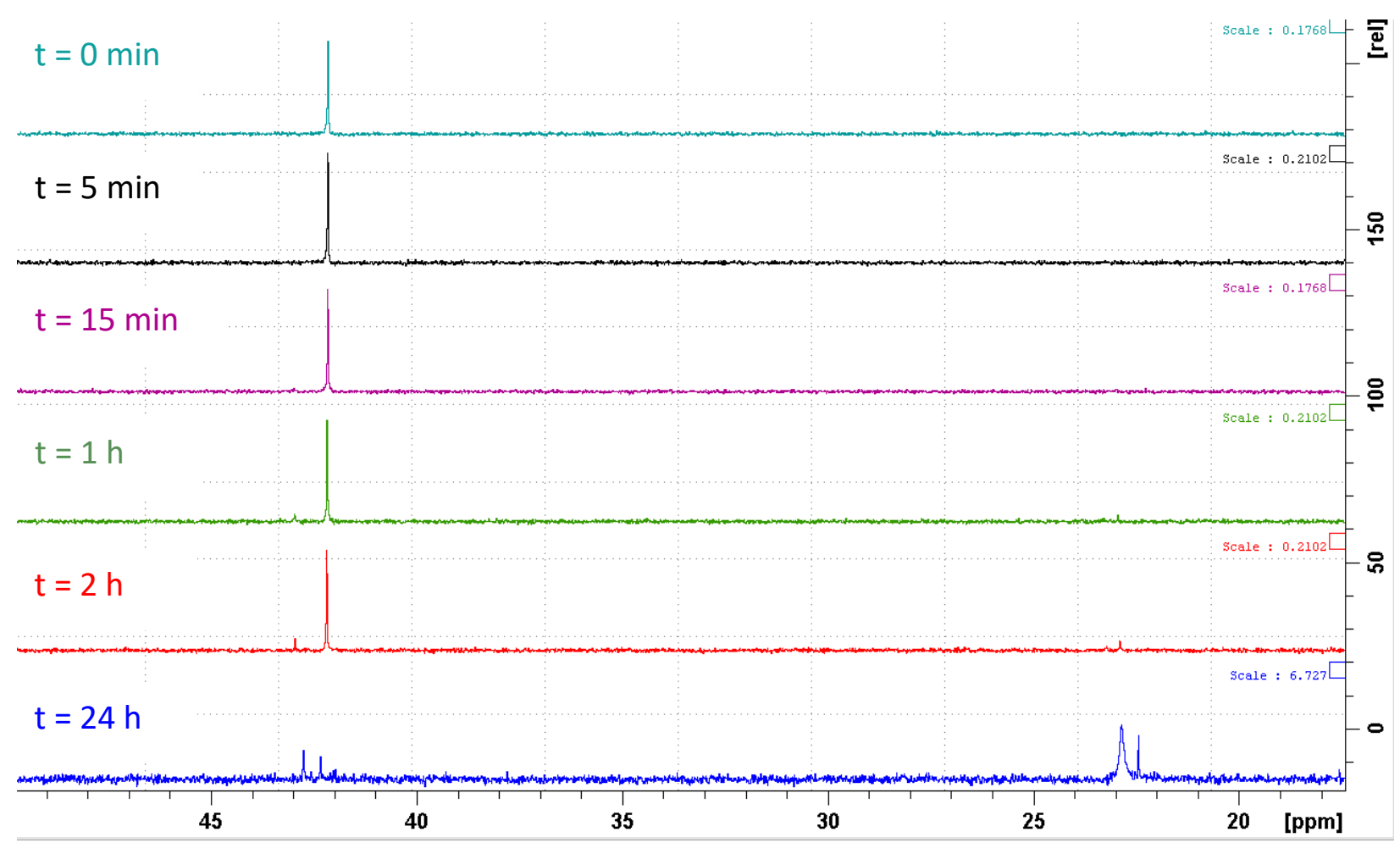

Figure S22: ${ }^{31} \mathrm{P}\left\{{ }^{1} \mathrm{H}\right\}$ NMR spectra of $L_{P=N 3} K(2)$ taken at different time intervals at $55{ }^{\circ} \mathrm{C}$ (benzene- $\left.d_{6}\right)$. 


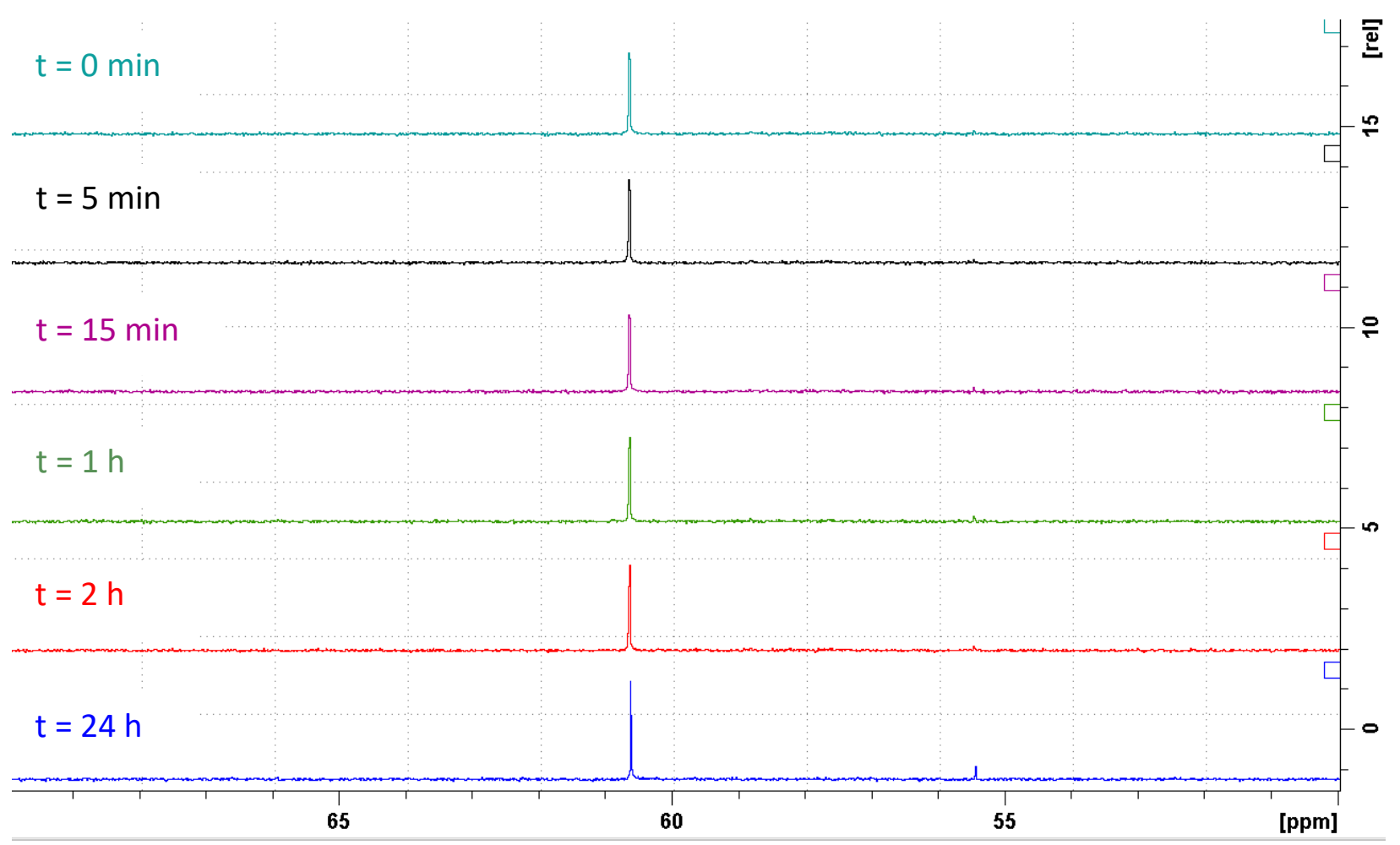

Figure S23: ${ }^{31} \mathrm{P}\left\{{ }^{1} \mathrm{H}\right\}$ NMR spectra of $\mathrm{L}_{\mathrm{P}=\mathrm{N} 3} \mathrm{ThCl}_{3}(\mathbf{3})$ taken at different time intervals at ambient temperature (benzene- $d_{6}$ ).

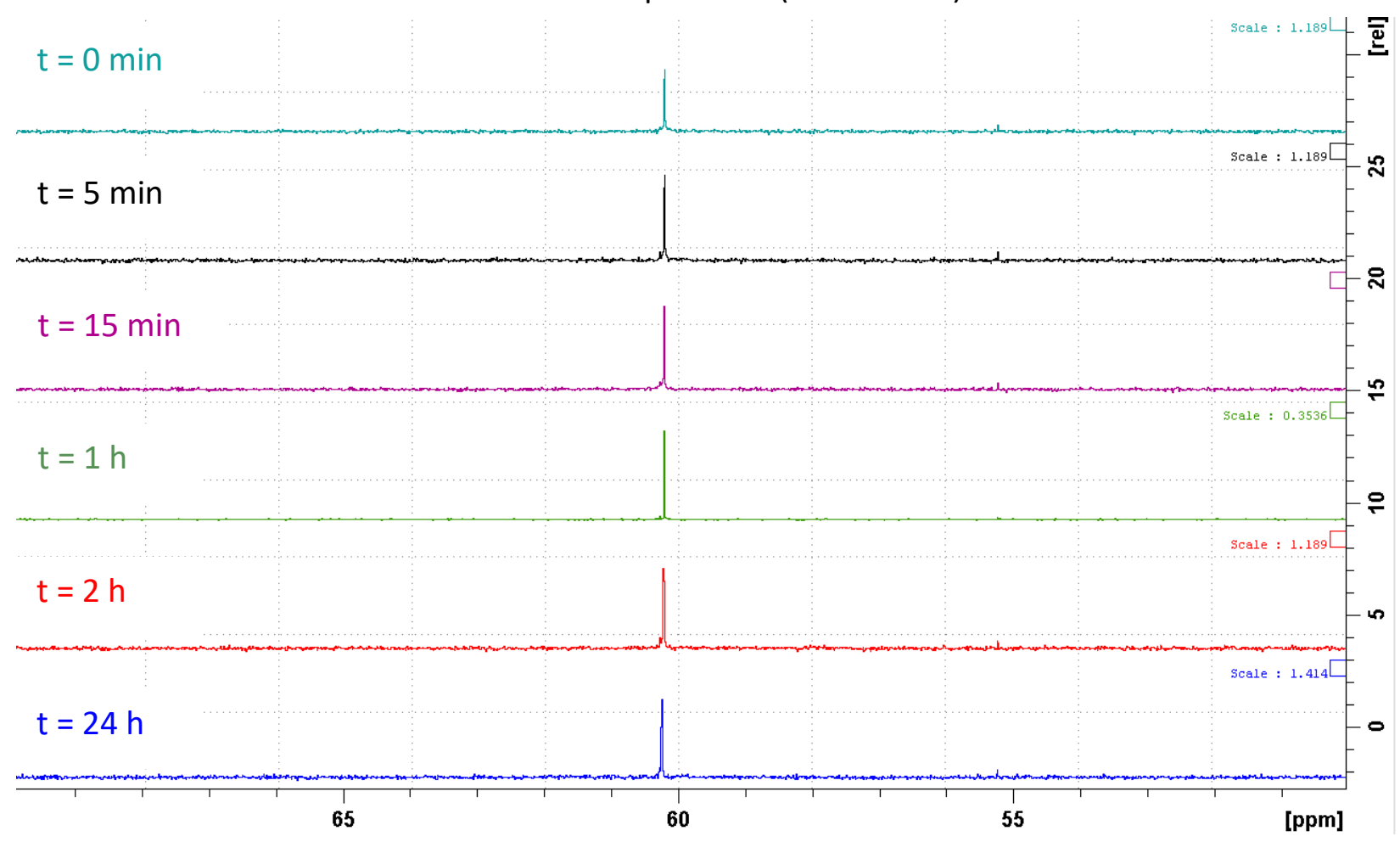

Figure S24: ${ }^{31} \mathrm{P}\left\{{ }^{1} \mathrm{H}\right\}$ NMR spectra of $\mathrm{L}_{\mathrm{P}=\mathrm{N} 3} \mathrm{ThCl}_{3}(\mathbf{3})$ taken at different time intervals at $55^{\circ} \mathrm{C}$ (benzene- $d_{6}$ ). 


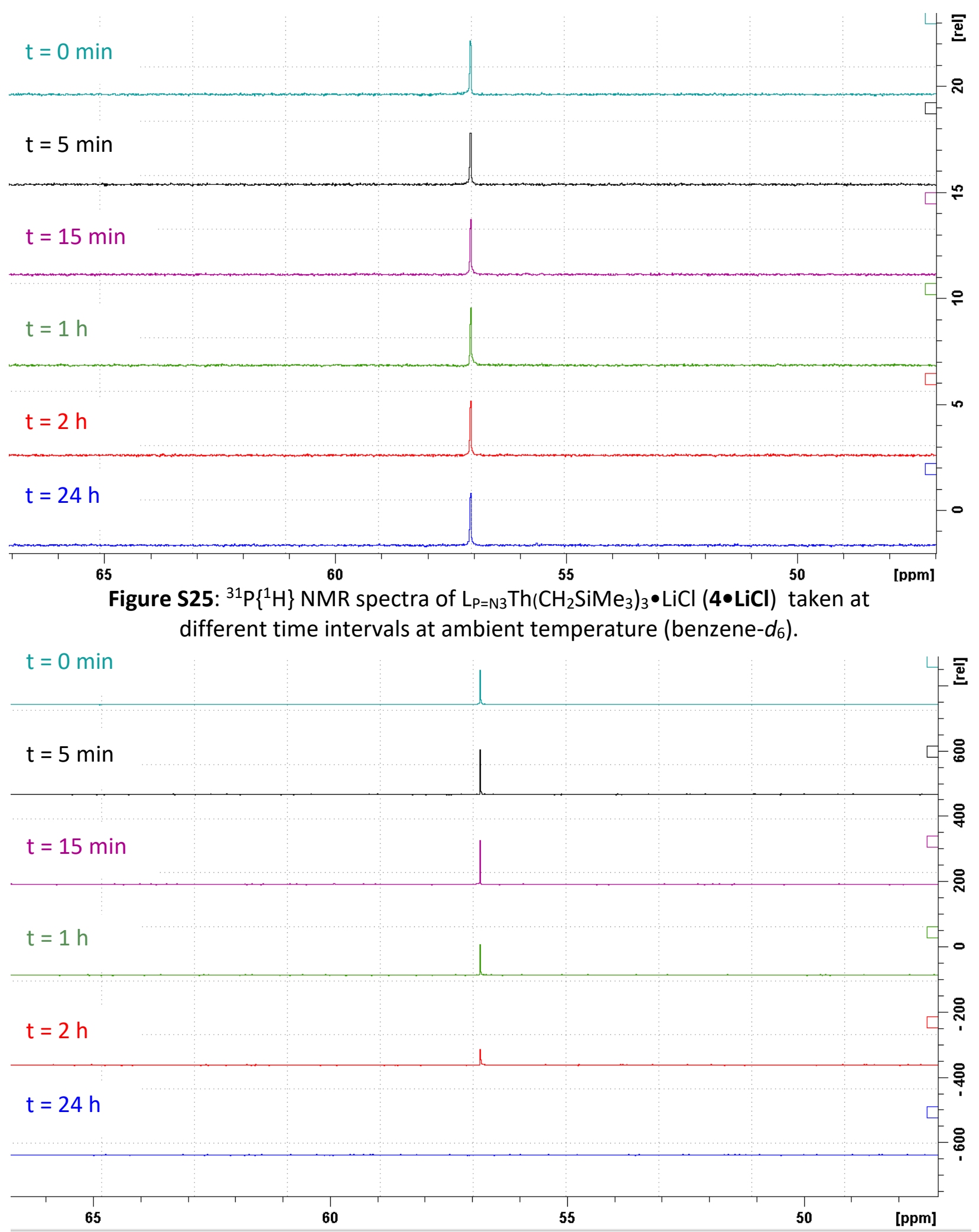

Figure S26: ${ }^{31} \mathrm{P}\left\{{ }^{1} \mathrm{H}\right\}$ NMR spectra of $\mathrm{L}_{\mathrm{P}=\mathrm{N} 3} \mathrm{Th}\left(\mathrm{CH}_{2} \mathrm{SiMe}_{3}\right)_{3} \bullet \mathrm{LiCl}(\mathbf{4} \bullet \mathrm{LiCl})$ taken at different time intervals at $55^{\circ} \mathrm{C}$ (benzene- $d_{6}$ ). 


\section{Crystallographic Details}

Unfortunately, single crystals of $\mathbf{2}$ grown in toluene, and $\mathbf{3}$ grown in benzene- $d_{6}$, succumb to rapid decomposition upon removal from the mother liquor, resulting in the crystals becoming covered with amorphic material. However, inner portions of the crystals remain intact, allowing for modest diffraction of X-ray radiation, despite interference from the polycrystalline material. The data is not ideal, however, and therefore discussions of the metrical parameters are not included in the body of the manuscript. The data set does, though, allow for qualitative discussions associated with the connectivity and geometry of complexes $\mathbf{2}$ and $\mathbf{3}$. The structures of $\mathbf{2}$ and $\mathbf{3}$ are depicted in Figures S27 and Figure S28, respectively.

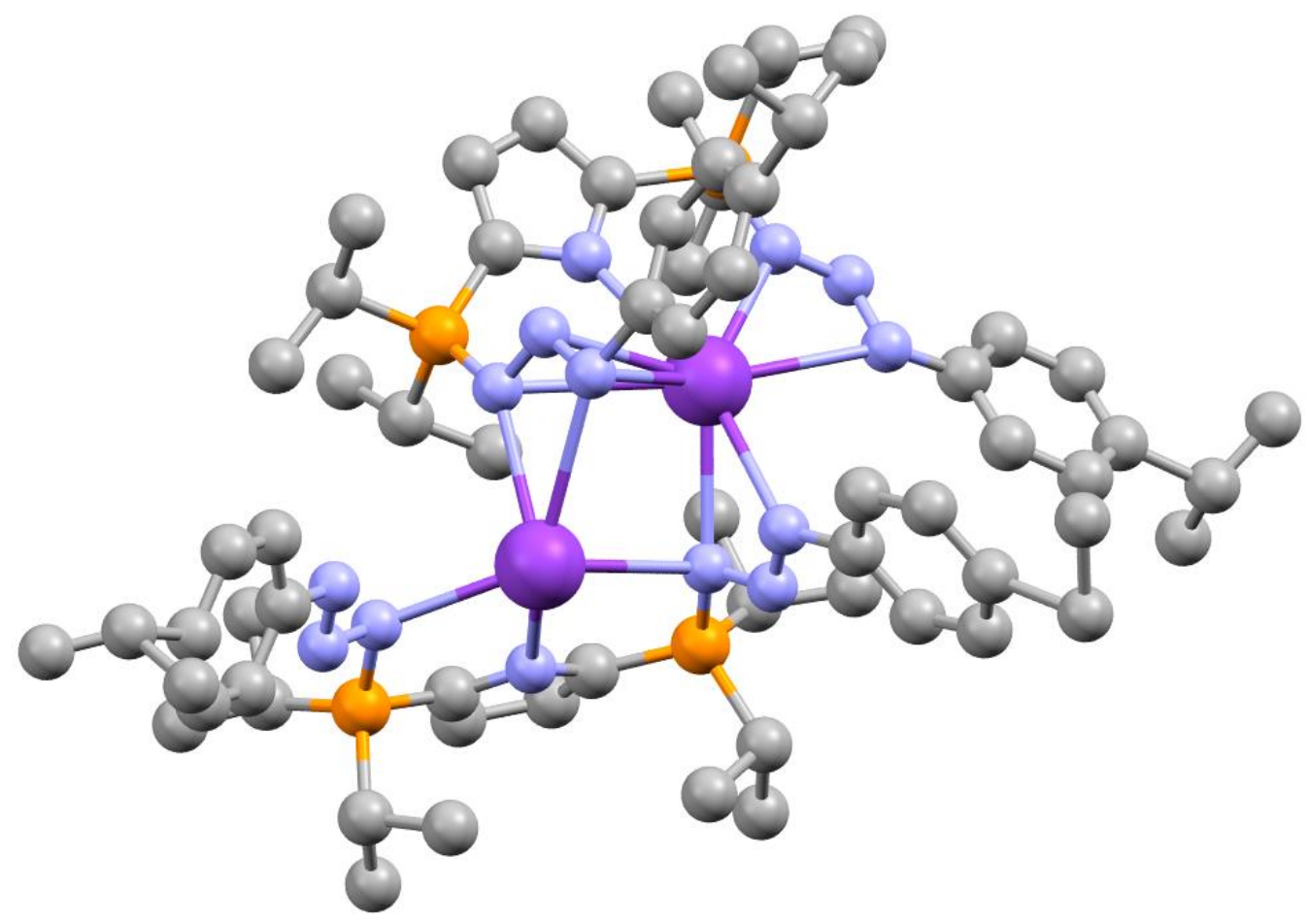

Figure S27. Connectivity structure of 2. Hydrogens have been removed for clarity. 


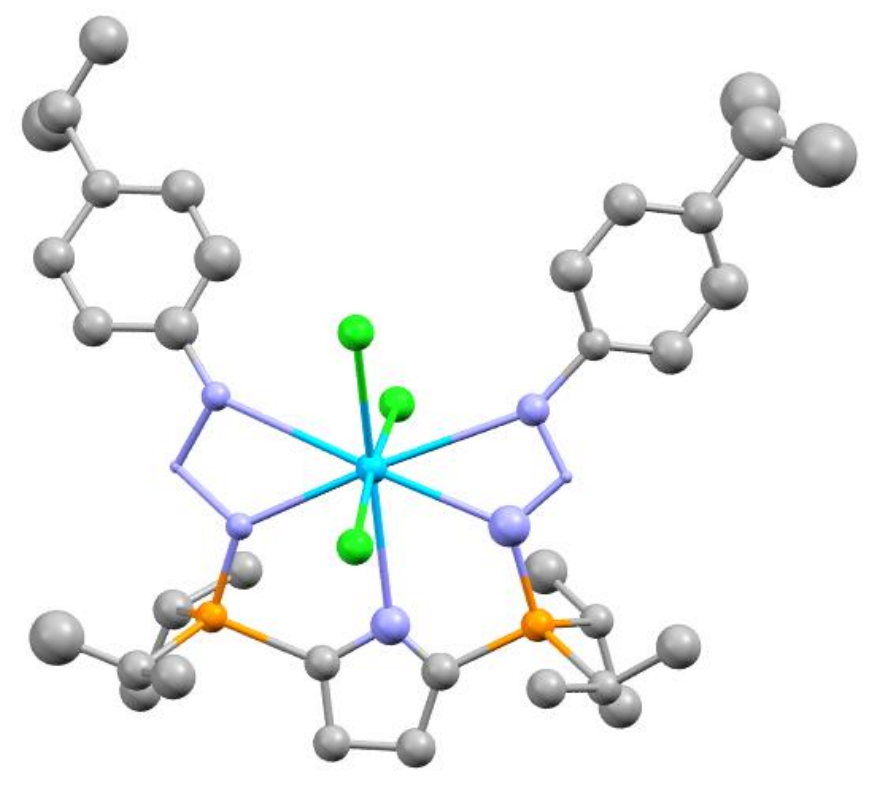

Figure S28. Connectivity structure of $\mathbf{3}$. Hydrogens have been removed for clarity. Only one of the two independent molecules in the asymmetric unit is shown.

Single crystals of complexes 4 and $\mathbf{6}$ were grown from pentane/toluene at $-35{ }^{\circ} \mathrm{C}$, and single crystals of ${ }^{5} \mathrm{C}_{7} \mathrm{H}_{8}$ were grown from a saturated solution of toluene at $-35^{\circ} \mathrm{C}$. Single crystals were coated in a dry Paratone ${ }^{\circledR}$ oil under an inert argon atmosphere. Crystals were mounted using MiTeGen Microloops ${ }^{\mathrm{TM}}$. Crystal data was collected on a Rigaku SuperNova, Dual, $\mathrm{Cu}$ at zero, Pilatus 200K diffractometer. Samples were cooled to $100 \mathrm{~K}$ using an Oxford Cryostream device. Data reduction was accomplished by the CrysAlis ${ }^{\text {Pro }}$ (version 1.171.38.43) software package. Absorption corrections were applied by multi-scan techniques and empirical absorption correction using spherical harmonics, implemented in SCALE3 ABSPACK scaling algorithm. Structures were solved in the Olex $2^{5}$ environment using intrinsic phasing and refined by full-matrix least squares method on $F^{2}$ using the SHELX software suite..$^{4-5}$ All non-hydrogen atoms were refined anisotropically, $\mathrm{C}-\mathrm{H}$ hydrogens were calculated and refined isotropically as a riding model. Summary of the crystallographic data can be found below in Table S1. 


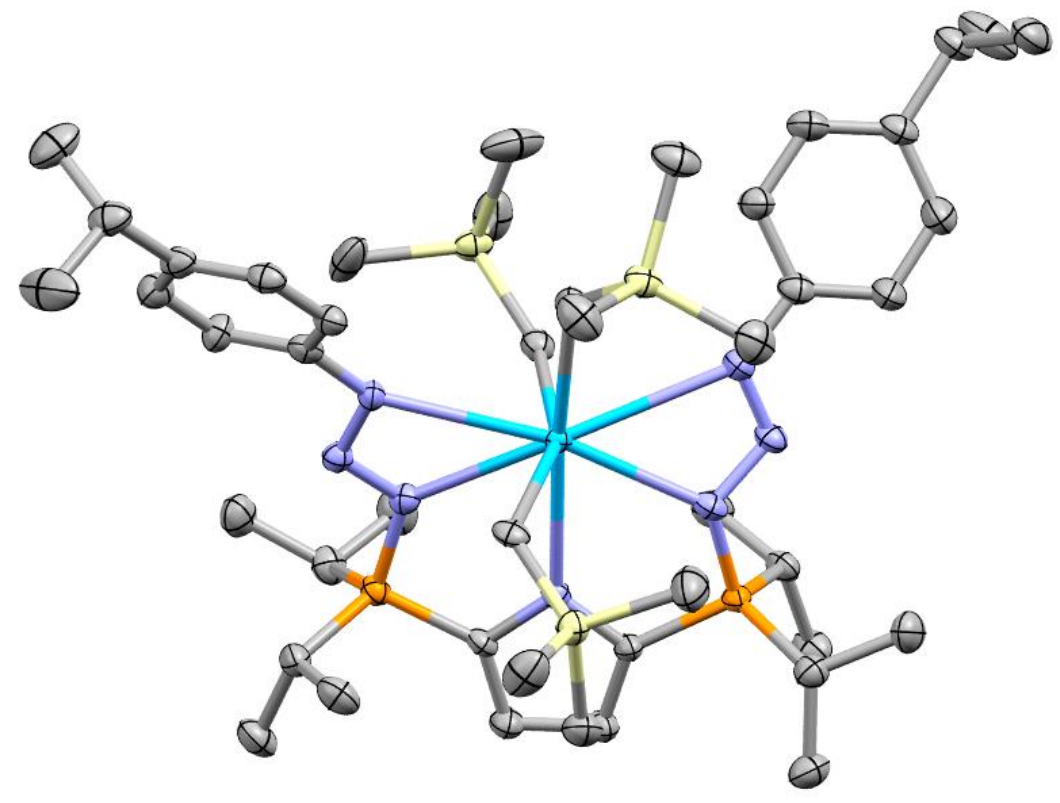

Figure S29. X-ray crystal structure of $\mathbf{4}$ with thermal ellipsoids drawn at 50\% probability. Hydrogens have been removed for clarity.

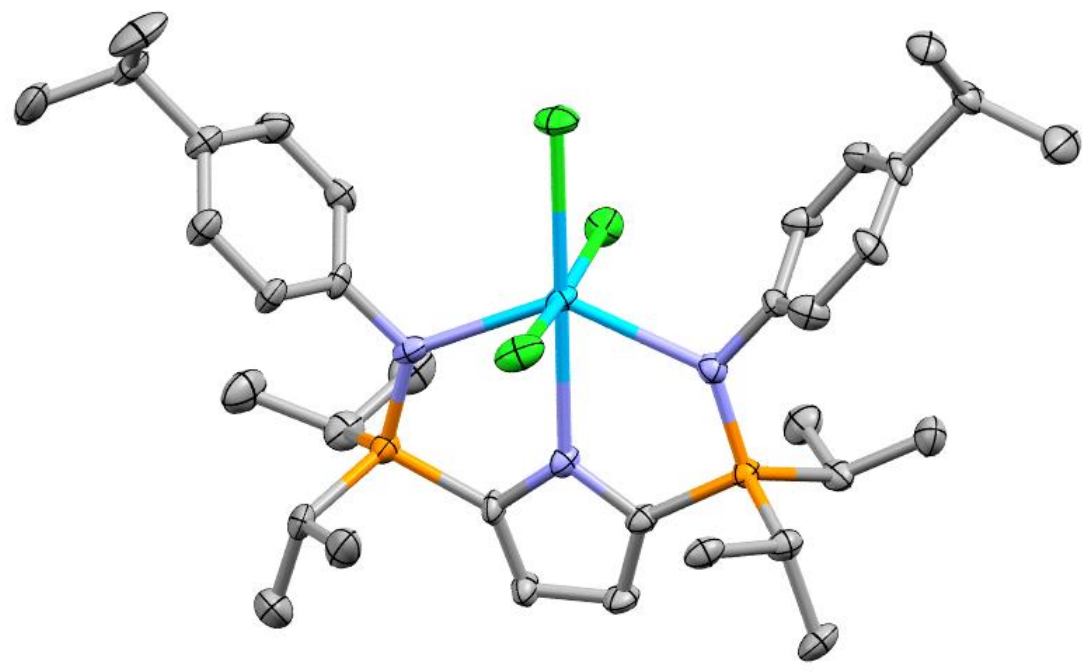

Figure S30. X-ray crystal structure of $\mathbf{5} \cdot \mathbf{C}_{7} \mathbf{H} \mathbf{8}$ with thermal ellipsoids drawn at $50 \%$ probability. Hydrogens and solvent molecule of crystallization have been removed for clarity. 


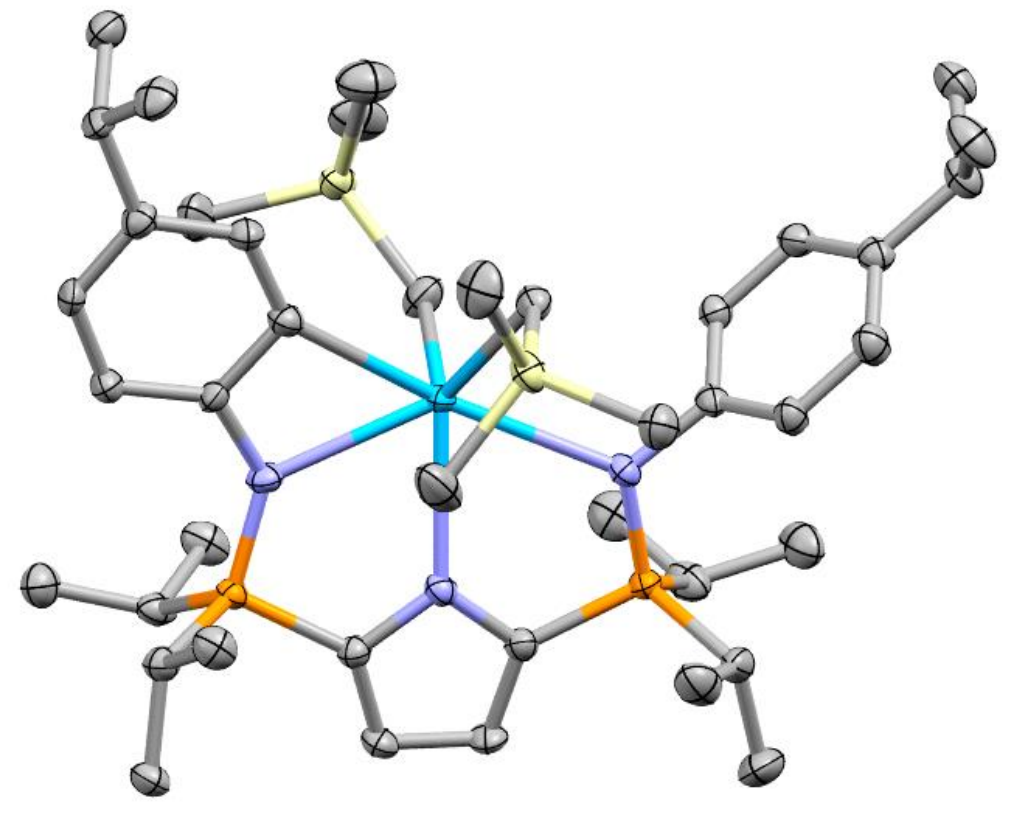

Figure S31. X-ray crystal structure of 6 with thermal ellipsoids drawn at 50\% probability. Hydrogens have been removed for clarity. 
Table S1. X-ray Crystallographic data and structure refinement for complexes 2, 3, 4, 5 and 6.

\begin{tabular}{|c|c|c|c|c|c|}
\hline & 2 & 3 & 4 & 5 & 6 \\
\hline CCDC \# & N/A & N/A & 1973166 & 1973168 & 1973167 \\
\hline Empirical formula & $\mathrm{C}_{67} \mathrm{H}_{97} \mathrm{~K}_{2} \mathrm{~N}_{14} \mathrm{P}_{4}$ & $\mathrm{C}_{68} \mathrm{H}_{102} \mathrm{Cl}_{6} \mathrm{~N}_{14} \mathrm{P}_{4} \mathrm{Th}_{2}$ & $\mathrm{C}_{46} \mathrm{H}_{85} \mathrm{~N}_{7} \mathrm{P}_{2} \mathrm{Si}_{3} \mathrm{Th}$ & $\mathrm{C}_{41} \mathrm{H}_{60} \mathrm{Cl}_{3} \mathrm{~N}_{3} \mathrm{P}_{2} \mathrm{Th}$ & $\mathrm{C}_{42} \mathrm{H}_{73} \mathrm{~N}_{3} \mathrm{P}_{2} \mathrm{Si}_{2} \mathrm{Th}$ \\
\hline Formula weight/g mol${ }^{-1}$ & 1300.66 & 1916.29 & 1114.45 & 995.25 & 970.19 \\
\hline Temperature/K & $145(1)$ & $120.01(10)$ & $99.9(4)$ & $100.01(17)$ & $100.00(10)$ \\
\hline Crystal system & monoclinic & orthorhombic & monoclinic & monoclinic & monoclinic \\
\hline \multirow{3}{*}{$\begin{array}{l}\text { Space group } \\
\mathrm{a} / \AA \\
\mathrm{b} / \AA\end{array}$} & $\mathrm{P} 2{ }_{1} / \mathrm{c}$ & Pna2 1 & $\mathrm{C} 2 / \mathrm{c}$ & $\mathrm{P} 2{ }_{1} / \mathrm{c}$ & $\mathrm{P} 2{ }_{1} / \mathrm{c}$ \\
\hline & $25.756(8)$ & $12.3671(2)$ & $38.1800(2)$ & $11.44990(10)$ & $12.25321(8)$ \\
\hline & $13.402(3)$ & $26.5522(5)$ & $12.01066(7)$ & $23.70340(10)$ & $19.54444(14)$ \\
\hline $\mathrm{c} / \AA$ & $24.793(8)$ & $26.8457(6)$ & $24.88322(15)$ & $17.73280(10)$ & $20.87594(14)$ \\
\hline$\alpha /^{\circ}$ & 90 & 90 & 90 & 90 & 90 \\
\hline$\beta /{ }^{\circ}$ & $106.25(3)$ & 90 & $101.1728(6)$ & $108.7210(10)$ & $97.9130(6)$ \\
\hline$\gamma /{ }^{\circ}$ & 90 & 90 & 90 & 90 & 90 \\
\hline Volume $/ \AA^{3}$ & $8216(4)$ & $8815.4(3)$ & 11194.37(12) & $4558.08(6)$ & 4951.81(6) \\
\hline $\mathrm{Z}$ & 4 & 4 & 8 & 4 & 4 \\
\hline$\rho_{\text {calc }} \mathrm{g} / \mathrm{cm}^{3}$ & 1.051 & 13.717 & 1.323 & 1.450 & 1.301 \\
\hline$\mu / \mathrm{mm}^{-1}$ & 2.085 & 128.268 & 10.010 & 13.051 & 10.989 \\
\hline $\mathrm{F}(000)$ & 2780.0 & 36100.0 & 4576.0 & 1992.0 & 1976.0 \\
\hline Crystal size $/ \mathrm{mm}^{3}$ & $0.2 \times 0.1 \times 0.02$ & $0.2 \times 0.2 \times 0.02$ & $0.35 \times 0.31 \times 0.25$ & $0.4 \times 0.2 \times 0.05$ & $0.15 \times 0.1 \times 0.02$ \\
\hline Radiation $(\AA)$ & $\mathrm{CuK} \alpha(\lambda=1.54184)$ & $\mathrm{CuK} \alpha(\lambda=1.54184)$ & $\mathrm{CuK} \alpha(\lambda=1.54184)$ & $\operatorname{CuK} \alpha(\lambda=1.54184)$ & $\mathrm{CuK} \alpha(\lambda=1.54184)$ \\
\hline $2 \Theta$ range for data collection ${ }^{\circ}$ & 7.504 to 103.954 & 8.548 to 160.35 & 7.242 to 160.772 & 6.45 to 160.4 & 7.284 to 160.65 \\
\hline Index ranges & $\begin{array}{l}-25 \leq \mathrm{h} \leq 26,-7 \leq \mathrm{k} \leq \\
10,-25 \leq 1 \leq 21\end{array}$ & $\begin{array}{l}-15 \leq \mathrm{h} \leq 15,-33 \leq \mathrm{k} \\
24,-34 \leq 1 \leq 34\end{array}$ & $\begin{array}{l}-48 \leq \mathrm{h} \leq 48,-14 \leq \mathrm{k} \\
14,-31 \leq 1 \leq 24\end{array}$ & $\begin{array}{l}-13 \leq \mathrm{h} \leq 14,-28 \leq \mathrm{k} \\
\leq 30,-22 \leq 1 \leq 22\end{array}$ & $\begin{array}{l}-15 \leq \mathrm{h} \leq 14,-22 \leq \mathrm{k} \leq \\
24,-26 \leq 1 \leq 26\end{array}$ \\
\hline \multirow{2}{*}{$\begin{array}{l}\text { Reflections collected } \\
\text { Independent reflections }\end{array}$} & 14731 & 23685 & 65766 & 28177 & 56770 \\
\hline & $\begin{array}{l}6994\left[R_{\text {int }}=0.2942,\right. \\
\left.R_{\text {sigma }}=0.4697\right]\end{array}$ & $\begin{array}{l}8689 \quad\left[R_{\text {int }}=0.1012\right. \\
\left.R_{\text {sigma }}=0.0667\right]\end{array}$ & $\begin{array}{l}12093\left[\mathrm{R}_{\text {int }}=0.0393\right. \\
\left.\mathrm{R}_{\text {sigma }}=0.0242\right]\end{array}$ & $\begin{array}{l}8728\left[R_{\text {int }}=0.0235\right. \\
\left.R_{\text {sigma }}=0.0214\right]\end{array}$ & $\begin{array}{l}10779\left[\mathrm{R}_{\text {int }}=0.0494,\right. \\
\left.\mathrm{R}_{\text {sigma }}=0.0340\right]\end{array}$ \\
\hline Data/restraints/parameters & $6994 / 0 / 322$ & $8689 / 1 / 81$ & $12093 / 6 / 577$ & $8728 / 0 / 464$ & $10779 / 4 / 477$ \\
\hline Goodness-of-fit on $\mathrm{F}^{2}(S)^{\mathrm{a}}$ & 1.185 & 6.696 & 1.177 & 1.072 & 1.156 \\
\hline Final $R$ indexes $[\mathrm{I}>=2 \sigma(\mathrm{I})]^{\mathrm{b}}$ & $\begin{array}{l}\mathrm{R}_{1}=0.1711 \\
\mathrm{wR}_{2}=0.3425\end{array}$ & $\begin{array}{l}\mathrm{R}_{1}=0.4198 \\
\mathrm{wR}_{2}=0.7399\end{array}$ & $\begin{array}{l}\mathrm{R}_{1}=0.0300 \\
\mathrm{wR}_{2}=0.0805\end{array}$ & $\begin{array}{l}\mathrm{R}_{1}=0.0248 \\
\mathrm{wR}_{2}=0.0655\end{array}$ & $\begin{array}{l}\mathrm{R}_{1}=0.0328 \\
\mathrm{wR}_{2}=0.0885\end{array}$ \\
\hline Final $\mathrm{R}$ indexes [all data] ${ }^{\mathrm{b}}$ & $\begin{array}{l}\mathrm{R}_{1}=0.3966 \\
\mathrm{wR}_{2}=0.4213\end{array}$ & $\begin{array}{l}\mathrm{R}_{1}=0.4403 \\
\mathrm{wR}_{2}=0.7622\end{array}$ & $\begin{array}{l}\mathrm{R}_{1}=0.0315 \\
\mathrm{wR}_{2}=0.0813\end{array}$ & $\begin{array}{l}\mathrm{R}_{1}=0.0259 \\
\mathrm{wR}_{2}=0.0679\end{array}$ & $\begin{array}{l}\mathrm{R}_{1}=0.0362 \\
\mathrm{wR}_{2}=0.0901\end{array}$ \\
\hline Largest diff. peak/hole / e $\AA^{-3}$ & $0.82 /-0.42$ & $65.17 /-14.62$ & $1.14 /-1.68$ & $0.81 /-1.17$ & $1.15 /-1.71$ \\
\hline
\end{tabular}

Programs for diffractometer operation, data collection, data reduction, and absorption correction were those supplied by Rigaku. a $S=\left[w\left(F_{\mathrm{o}}^{2}-F_{\mathrm{c}}^{2}\right)^{2} /(n-p)\right]^{1 / 2}\left(n=\right.$ number of data; $p=$ number of parameters varied; $w=\left[\square^{2}\left(F_{\mathrm{o}}^{2}\right)+(0.0540 P)^{2}+22.8160 P\right]^{-1}$ where $\left.P=\left[\operatorname{Max}\left(F_{\mathrm{o}}^{2}, 0\right)+2 F_{\mathrm{c}}{ }^{2}\right] / 3\right)$.

${ }^{\mathrm{b}} \mathrm{R}_{1}=\square|| F_{\mathrm{o}}|-| F_{\mathrm{c}}|| \square\left|F_{\mathrm{o}}\right| ; w \mathrm{R}_{2}=\left[\square w\left(F_{\mathrm{o}}{ }^{2}-F_{\mathrm{c}}{ }^{2}\right)^{2} / \square w\left(F_{\mathrm{o}}{ }^{4}\right)\right]^{1 / 2}$ 
Table S2. Fractional Atomic Coordinates $\left(\times 10^{4}\right)$ and Equivalent Isotropic Displacement Parameters $\left(\AA^{2} \times 10^{3}\right)$ for 4 . $U_{\text {eq }}$ is defined as $1 / 3$ of of the trace of the orthogonalized $U_{\text {IJ }}$ tensor.

\begin{tabular}{|c|c|c|c|c|}
\hline Atom & $\mathbf{x}$ & $\mathbf{y}$ & $\mathbf{z}$ & $\mathrm{U}(\mathbf{e q})$ \\
\hline Th1 & $3864.4(2)$ & $2789.0(2)$ & $5479.3(2)$ & $18.34(5)$ \\
\hline $\mathrm{P} 1$ & $3133.8(2)$ & $4911.8(7)$ & $4850.7(4)$ & $21.99(17)$ \\
\hline $\mathrm{P} 2$ & $4263.4(2)$ & $3195.1(7)$ & $4181.8(3)$ & $20.33(16)$ \\
\hline Si1 & $4183.4(3)$ & $81.8(8)$ & $6378.4(4)$ & $27.4(2)$ \\
\hline $\mathrm{Si} 2$ & $4190.9(3)$ & $5202.5(8)$ & $6622.7(4)$ & $27.6(2)$ \\
\hline $\mathrm{Si3}$ & $3310.6(3)$ & $570.1(9)$ & 4499.1(4) & 27.1(2) \\
\hline N1 & $3682.8(7)$ & 3914(2) & $4522.6(12)$ & $21.2(6)$ \\
\hline N2 & $3330.1(8)$ & $4127(2)$ & $5365.1(12)$ & $22.2(6)$ \\
\hline N3 & $3137.0(8)$ & $3910(2)$ & $5753.8(12)$ & $22.2(6)$ \\
\hline N4 & $3301.2(7)$ & $3182(2)$ & $6083.0(12)$ & $21.2(6)$ \\
\hline N5 & $4283.2(8)$ & 2647(3) & $4798.5(12)$ & $24.6(6)$ \\
\hline N6 & $4623.8(8)$ & $2498(3)$ & 5078.2(12) & $24.5(6)$ \\
\hline N7 & $4618.4(8)$ & $2269(2)$ & $5573.8(13)$ & $24.1(6)$ \\
\hline $\mathrm{C} 1$ & $3392.2(9)$ & $4592(3)$ & $4355.6(14)$ & $22.6(7)$ \\
\hline $\mathrm{C} 2$ & $3368.9(10)$ & $4954(3)$ & $3818.8(15)$ & $27.8(7)$ \\
\hline $\mathrm{C} 3$ & $3660.2(10)$ & $4483(3)$ & $3638.2(15)$ & $26.6(7)$ \\
\hline $\mathrm{C} 4$ & $3843.2(9)$ & $3860(3)$ & 4074.2(13) & $20.8(6)$ \\
\hline $\mathrm{C} 5$ & $3173.8(10)$ & $6401(3)$ & 4999.2(16) & $29.6(8)$ \\
\hline C6 & $3567.7(11)$ & $6740(3)$ & $5150(2)$ & $38.0(9)$ \\
\hline $\mathrm{C} 7$ & $2968.9(12)$ & $6721(4)$ & $5449.5(18)$ & $36.9(9)$ \\
\hline $\mathrm{C} 8$ & 2662.3(9) & $4565(3)$ & $4630.6(15)$ & $27.4(7)$ \\
\hline C9 & $2623.3(11)$ & $3380(4)$ & $4410.5(18)$ & $35.4(9)$ \\
\hline $\mathrm{C} 10$ & $2463.1(11)$ & $5387(4)$ & $4211.4(19)$ & $38.3(9)$ \\
\hline C11 & $4614.4(9)$ & 4241(3) & $4208.9(15)$ & $25.6(7)$ \\
\hline $\mathrm{C} 12$ & $4564.2(11)$ & $5136(3)$ & $4624.7(17)$ & $33.6(8)$ \\
\hline $\mathrm{C} 13$ & $4622.2(11)$ & $4770(4)$ & $3648.6(16)$ & $32.5(8)$ \\
\hline C14 & $4281.4(11)$ & 2111(3) & $3674.2(15)$ & 27.1(7) \\
\hline $\mathrm{C} 15$ & $4660.8(12)$ & $1632(4)$ & $3734.3(18)$ & $37.7(9)$ \\
\hline C16 & $4116.9(13)$ & $2449(4)$ & $3084.6(16)$ & $35.3(9)$ \\
\hline $\mathrm{C} 17$ & $4962.8(10)$ & $2114(3)$ & $5907.9(15)$ & $26.4(7)$ \\
\hline $\mathrm{C} 18$ & $5267.1(11)$ & $1873(4)$ & $5704.9(17)$ & 37.1(9) \\
\hline C19 & $5593.6(12)$ & 1772(4) & $6062.8(18)$ & $41.0(10)$ \\
\hline $\mathrm{C} 20$ & $5622.2(10)$ & $1879(3)$ & 6631.1(16) & $30.1(8)$ \\
\hline $\mathrm{C} 21$ & $5315.2(11)$ & $2082(3)$ & $6824.2(16)$ & $30.3(8)$ \\
\hline $\mathrm{C} 22$ & 4988.1(11) & $2208(3)$ & $6472.7(16)$ & $29.1(8)$ \\
\hline $\mathrm{C} 23$ & $5982.8(11)$ & $1787(4)$ & $7022.0(17)$ & $33.2(8)$ \\
\hline $\mathrm{C} 24$ & $6229.1(14)$ & $2746(4)$ & $6940(3)$ & $56.8(15)$ \\
\hline $\mathrm{C} 25$ & $6158.8(12)$ & $659(4)$ & 6976.1(19) & $42.5(10)$ \\
\hline $\mathrm{C} 26$ & $3114.5(10)$ & $2884(3)$ & $6501.8(15)$ & 24.2(7) \\
\hline $\mathrm{C} 27$ & $3138.6(9)$ & $1782(3)$ & $6683.9(14)$ & 25.3(7) \\
\hline $\mathrm{C} 28$ & $2943.7(10)$ & $1439(3)$ & $7076.7(15)$ & $28.1(7)$ \\
\hline
\end{tabular}




\begin{tabular}{|l|c|c|c|c|}
\hline C29 & $2729.6(10)$ & $2168(3)$ & $7300.5(16)$ & $28.6(8)$ \\
\hline C30 & $2714.4(11)$ & $3277(4)$ & $7117.0(16)$ & $32.6(8)$ \\
\hline C31 & $2903.5(10)$ & $3630(3)$ & $6726.2(15)$ & $28.2(7)$ \\
\hline C32 & $2515.3(12)$ & $1761(4)$ & $7714.4(16)$ & $35.8(9)$ \\
\hline C33 & $2132.6(14)$ & $1543(7)$ & $7440(2)$ & $68.9(19)$ \\
\hline C34 & $2537.1(17)$ & $2539(5)$ & $8198(2)$ & $51.2(12)$ \\
\hline C35 & $4038.0(10)$ & $1554(3)$ & $6305.8(15)$ & $25.4(7)$ \\
\hline C36 & $4158.0(10)$ & $4486(3)$ & $5960.2(15)$ & $27.9(7)$ \\
\hline C37 & $3356.6(10)$ & $1406(3)$ & $5116.8(14)$ & $25.8(7)$ \\
\hline C38 & $3723.9(14)$ & $-289(5)$ & $4537(2)$ & $48.6(12)$ \\
\hline C39 & $3240.0(11)$ & $1395(4)$ & $3844.9(16)$ & $33.4(8)$ \\
\hline C40 & $2932.9(13)$ & $-478(4)$ & $4394(2)$ & $45.3(11)$ \\
\hline C41 & $3746.4(12)$ & $5600(5)$ & $6770(2)$ & $49.1(13)$ \\
\hline C42 & $4466.5(12)$ & $6508(4)$ & $6679(2)$ & $44.1(11)$ \\
\hline C43 & $4424.2(18)$ & $4263(4)$ & $7177.5(19)$ & $59.5(16)$ \\
\hline C44 & $4506.7(12)$ & $-230(4)$ & $7036.8(18)$ & $40.6(10)$ \\
\hline C45 & $4426.5(13)$ & $-396(4)$ & $5832.5(19)$ & $41.2(10)$ \\
\hline C46 & $3777.7(12)$ & $-827(4)$ & $6354(2)$ & $42.4(10)$ \\
\hline
\end{tabular}

Table S3. Anisotropic Displacement Parameters $\left(\AA^{2} \times 10^{3}\right)$ for 4. The Anisotropic displacement factor exponent takes the form: $-2 \pi^{2}\left[h^{2} a^{* 2} U_{11}+2 h k a * b * U_{12}+\ldots\right]$.

\begin{tabular}{|c|c|c|c|c|c|c|}
\hline Atom & $\mathbf{U}_{\mathbf{1 1}}$ & $\mathbf{U}_{\mathbf{2 2}}$ & $\mathbf{U}_{\mathbf{3 3}}$ & $\mathbf{U}_{\mathbf{2 3}}$ & $\mathbf{U}_{\mathbf{1 3}}$ & $\mathbf{U}_{\mathbf{1 2}}$ \\
\hline Th1 & $19.04(7)$ & $18.96(7)$ & $16.33(6)$ & $0.77(4)$ & $1.71(4)$ & $0.97(4)$ \\
\hline P1 & $21.1(4)$ & $20.9(4)$ & $23.2(4)$ & $2.0(3)$ & $2.3(3)$ & $4.1(3)$ \\
\hline P2 & $19.9(4)$ & $23.3(4)$ & $17.7(4)$ & $2.4(3)$ & $3.3(3)$ & $1.1(3)$ \\
\hline Si1 & $30.5(5)$ & $21.6(5)$ & $27.9(5)$ & $1.9(4)$ & $0.0(4)$ & $1.3(4)$ \\
\hline Si2 & $34.4(5)$ & $23.3(5)$ & $23.5(4)$ & $-2.7(4)$ & $1.5(4)$ & $-3.6(4)$ \\
\hline Si3 & $27.2(5)$ & $25.6(5)$ & $28.6(5)$ & $-1.1(4)$ & $5.9(4)$ & $-0.2(4)$ \\
\hline N1 & $18.8(13)$ & $20.6(14)$ & $22.9(13)$ & $-1.3(11)$ & $1.4(11)$ & $0.8(11)$ \\
\hline N2 & $22.8(14)$ & $22.2(14)$ & $21.5(13)$ & $1.5(11)$ & $4.5(11)$ & $-1.4(11)$ \\
\hline N3 & $22.9(14)$ & $22.2(14)$ & $21.6(13)$ & $-0.6(11)$ & $4.5(11)$ & $-0.2(11)$ \\
\hline N4 & $18.6(13)$ & $24.3(15)$ & $21.5(13)$ & $-1.6(11)$ & $6.3(11)$ & $1.6(11)$ \\
\hline N5 & $22.9(14)$ & $25.3(15)$ & $22.8(14)$ & $3.1(11)$ & $-2.5(11)$ & $2.8(12)$ \\
\hline N6 & $23.8(14)$ & $25.0(14)$ & $23.3(14)$ & $4.9(12)$ & $1.0(12)$ & $2.6(12)$ \\
\hline N7 & $21.9(14)$ & $26.3(16)$ & $23.1(15)$ & $1.2(11)$ & $2.2(12)$ & $2.6(11)$ \\
\hline C1 & $19.5(15)$ & $22.7(16)$ & $23.6(16)$ & $2.9(13)$ & $-1.0(12)$ & $3.8(13)$ \\
\hline C2 & $26.8(17)$ & $31.1(19)$ & $24.7(17)$ & $5.5(14)$ & $2.9(14)$ & $5.3(15)$ \\
\hline C3 & $25.1(17)$ & $30.5(19)$ & $23.6(16)$ & $3.6(14)$ & $3.4(13)$ & $1.4(14)$ \\
\hline C4 & $19.8(15)$ & $25.4(17)$ & $17.5(14)$ & $2.5(12)$ & $4.3(12)$ & $1.4(13)$ \\
\hline C5 & $31.9(19)$ & $22.7(18)$ & $34.6(19)$ & $2.6(15)$ & $7.3(15)$ & $4.1(14)$ \\
\hline C6 & $36(2)$ & $26(2)$ & $53(3)$ & $-2.6(18)$ & $8.9(19)$ & $-3.5(16)$ \\
\hline
\end{tabular}




\begin{tabular}{|c|c|c|c|c|c|c|}
\hline $\mathrm{C} 7$ & $38(2)$ & $31(2)$ & $42(2)$ & $-8.0(17)$ & $9.1(18)$ & $4.4(17)$ \\
\hline $\mathrm{C} 8$ & $22.2(16)$ & $33(2)$ & $26.6(17)$ & $1.4(15)$ & $4.1(13)$ & $4.6(14)$ \\
\hline C9 & 28.2(19) & $34(2)$ & $40(2)$ & $4.1(17)$ & $-3.3(16)$ & $-2.6(16)$ \\
\hline $\mathrm{C} 10$ & $28.6(19)$ & $38(2)$ & $44(2)$ & $-0.1(19)$ & $-5.2(17)$ & $8.2(17)$ \\
\hline $\mathrm{C} 11$ & $20.0(16)$ & $29.5(18)$ & 26.1(17) & $3.5(14)$ & $1.7(13)$ & $-1.2(14)$ \\
\hline $\mathrm{C} 12$ & $33.4(19)$ & $32(2)$ & $35(2)$ & $0.9(16)$ & $4.0(16)$ & $-8.2(16)$ \\
\hline C13 & $30.5(19)$ & $34(2)$ & $32.6(19)$ & $11.3(16)$ & $5.8(15)$ & $-3.3(16)$ \\
\hline $\mathrm{C} 14$ & $32.4(19)$ & $24.9(18)$ & $24.4(17)$ & $0.7(14)$ & $6.0(15)$ & $1.1(14)$ \\
\hline $\mathrm{C} 15$ & $38(2)$ & $36(2)$ & $39(2)$ & $-4.2(18)$ & $7.9(17)$ & $10.1(18)$ \\
\hline $\mathrm{C} 16$ & $47(2)$ & $35(2)$ & $23.9(18)$ & $-1.6(16)$ & $6.5(17)$ & $2.1(19)$ \\
\hline $\mathrm{C} 17$ & $29.3(18)$ & $23.4(17)$ & $24.4(17)$ & $3.8(13)$ & $0.3(14)$ & $0.8(14)$ \\
\hline $\mathrm{C} 18$ & $30(2)$ & $53(3)$ & $26.4(19)$ & $3.9(18)$ & $1.5(15)$ & $13.8(18)$ \\
\hline C19 & $32(2)$ & $56(3)$ & $35(2)$ & $5(2)$ & $5.9(17)$ & $13(2)$ \\
\hline $\mathrm{C} 20$ & $29.1(18)$ & $28.7(19)$ & $29.0(18)$ & $4.3(15)$ & $-2.8(15)$ & $4.1(15)$ \\
\hline $\mathrm{C} 21$ & $35(2)$ & $29.5(19)$ & $23.0(17)$ & $0.9(14)$ & $-2.2(15)$ & $-2.6(15)$ \\
\hline $\mathrm{C} 22$ & $29.7(19)$ & $32(2)$ & $24.5(18)$ & $0.9(14)$ & $3.9(15)$ & $-0.5(15)$ \\
\hline $\mathrm{C} 23$ & $28.2(19)$ & $34(2)$ & $33(2)$ & $4.0(16)$ & $-3.7(15)$ & $5.6(16)$ \\
\hline $\mathrm{C} 24$ & $36(2)$ & $50(3)$ & $75(4)$ & $26(3)$ & $-15(2)$ & $-10(2)$ \\
\hline $\mathrm{C} 25$ & $38(2)$ & $45(3)$ & $39(2)$ & $0.5(19)$ & $-6.0(18)$ & $13.8(19)$ \\
\hline $\mathrm{C} 26$ & $23.9(17)$ & $24.7(17)$ & $21.7(16)$ & $0.4(13)$ & $-1.6(13)$ & $-1.7(13)$ \\
\hline C27 & $23.6(16)$ & $27.8(18)$ & $23.3(16)$ & $2.2(14)$ & $1.6(13)$ & $-1.2(14)$ \\
\hline $\mathrm{C} 28$ & $28.0(17)$ & $30.4(19)$ & $24.0(17)$ & $5.5(14)$ & $0.6(14)$ & $-4.1(15)$ \\
\hline $\mathrm{C} 29$ & $25.9(18)$ & $36(2)$ & $23.4(17)$ & $1.3(14)$ & $3.7(14)$ & $-6.1(15)$ \\
\hline C30 & $37(2)$ & $32(2)$ & $29.7(19)$ & $-0.9(16)$ & $8.1(16)$ & $1.5(16)$ \\
\hline C31 & $32.6(19)$ & $26.5(18)$ & $28.2(18)$ & $-1.1(14)$ & $12.8(15)$ & $0.2(15)$ \\
\hline C32 & $42(2)$ & $41(2)$ & $25.3(18)$ & $4.1(17)$ & $7.6(16)$ & $-6.6(18)$ \\
\hline C33 & $44(3)$ & $118(6)$ & $43(3)$ & $19(3)$ & $6(2)$ & $-30(3)$ \\
\hline C34 & $78(4)$ & $47(3)$ & $34(2)$ & $0(2)$ & $23(2)$ & $-4(3)$ \\
\hline $\mathrm{C} 35$ & $23.7(16)$ & $25.0(17)$ & $27.6(17)$ & $-3.3(14)$ & $5.0(14)$ & $-1.2(14)$ \\
\hline C36 & $25.7(17)$ & $30.2(19)$ & $27.0(17)$ & $3.5(15)$ & $3.1(14)$ & $4.8(15)$ \\
\hline C37 & $27.6(17)$ & $25.5(18)$ & $22.3(16)$ & $7.5(13)$ & $-0.2(13)$ & $1.2(14)$ \\
\hline C38 & $48(3)$ & $55(3)$ & $46(3)$ & $9(2)$ & $17(2)$ & $20(2)$ \\
\hline C39 & $36(2)$ & $35(2)$ & $26.9(18)$ & $-0.6(16)$ & $1.6(15)$ & $-0.8(17)$ \\
\hline $\mathrm{C} 40$ & $51(3)$ & $41(2)$ & $47(3)$ & $-13(2)$ & $18(2)$ & $-17(2)$ \\
\hline $\mathrm{C} 41$ & $42(2)$ & 69(3) & $42(2)$ & $-29(2)$ & $20(2)$ & $-15(2)$ \\
\hline $\mathrm{C} 42$ & $40(2)$ & $32(2)$ & $63(3)$ & $-14(2)$ & $17(2)$ & $-7.2(18)$ \\
\hline $\mathrm{C} 43$ & $104(5)$ & $41(3)$ & $26(2)$ & $-5.8(19)$ & $-8(2)$ & $8(3)$ \\
\hline $\mathrm{C} 44$ & $46(2)$ & $30(2)$ & $38(2)$ & $5.5(17)$ & $-8.5(19)$ & $3.9(18)$ \\
\hline $\mathrm{C} 45$ & $47(2)$ & $29(2)$ & $47(2)$ & $-3.9(18)$ & $6(2)$ & $5.4(18)$ \\
\hline $\mathrm{C} 46$ & $37(2)$ & $29(2)$ & $59(3)$ & $5.2(19)$ & $3(2)$ & $-2.7(17)$ \\
\hline
\end{tabular}


Table S4. Bond Lengths for 4.

\begin{tabular}{|c|c|c|c|c|c|}
\hline Atom & Atom & Length/Å & Atom & Atom & Length/Å \\
\hline Th1 & N1 & $2.708(3)$ & N5 & N6 & $1.362(4)$ \\
\hline Th1 & N2 & $2.569(3)$ & N6 & N7 & $1.268(4)$ \\
\hline Th1 & N4 & $2.891(3)$ & N7 & $\mathrm{C} 17$ & $1.425(5)$ \\
\hline Th1 & N5 & $2.551(3)$ & $\mathrm{C} 1$ & $\mathrm{C} 2$ & $1.391(5)$ \\
\hline Th1 & N7 & $2.910(3)$ & $\mathrm{C} 2$ & $\mathrm{C} 3$ & $1.397(5)$ \\
\hline Th1 & C35 & $2.518(4)$ & $\mathrm{C} 3$ & $\mathrm{C} 4$ & $1.390(5)$ \\
\hline Th1 & C36 & $2.514(4)$ & $\mathrm{C} 5$ & C6 & $1.533(6)$ \\
\hline Th1 & C37 & $2.580(4)$ & $\mathrm{C} 5$ & $\mathrm{C} 7$ & $1.534(6)$ \\
\hline $\mathrm{P} 1$ & N2 & $1.648(3)$ & $\mathrm{C} 8$ & C9 & $1.522(6)$ \\
\hline $\mathrm{P} 1$ & $\mathrm{C} 1$ & $1.764(4)$ & $\mathrm{C} 8$ & $\mathrm{C} 10$ & $1.527(5)$ \\
\hline $\mathrm{P} 1$ & $\mathrm{C} 5$ & $1.827(4)$ & C11 & $\mathrm{C} 12$ & $1.530(6)$ \\
\hline $\mathrm{P} 1$ & $\mathrm{C} 8$ & $1.825(4)$ & C11 & C13 & $1.538(5)$ \\
\hline $\mathrm{P} 2$ & N5 & $1.658(3)$ & C14 & $\mathrm{C} 15$ & $1.539(6)$ \\
\hline $\mathrm{P} 2$ & $\mathrm{C} 4$ & $1.765(3)$ & C14 & C16 & $1.534(5)$ \\
\hline $\mathrm{P} 2$ & C11 & $1.828(4)$ & C17 & $\mathrm{C} 18$ & $1.384(6)$ \\
\hline $\mathrm{P} 2$ & $\mathrm{C} 14$ & $1.825(4)$ & $\mathrm{C} 17$ & $\mathrm{C} 22$ & $1.395(5)$ \\
\hline Si1 & $\mathrm{C} 35$ & $1.852(4)$ & $\mathrm{C} 18$ & C19 & $1.390(6)$ \\
\hline Si1 & $\mathrm{C} 44$ & $1.888(4)$ & C19 & $\mathrm{C} 20$ & $1.403(6)$ \\
\hline Si1 & $\mathrm{C} 45$ & $1.878(5)$ & $\mathrm{C} 20$ & $\mathrm{C} 21$ & $1.372(6)$ \\
\hline Si1 & $\mathrm{C} 46$ & $1.886(4)$ & $\mathrm{C} 20$ & $\mathrm{C} 23$ & $1.528(5)$ \\
\hline $\mathrm{Si} 2$ & C36 & $1.842(4)$ & $\mathrm{C} 21$ & $\mathrm{C} 22$ & $1.387(5)$ \\
\hline $\mathrm{Si} 2$ & $\mathrm{C} 41$ & $1.866(5)$ & $\mathrm{C} 23$ & $\mathrm{C} 24$ & $1.526(6)$ \\
\hline $\mathrm{Si} 2$ & $\mathrm{C} 42$ & $1.878(5)$ & $\mathrm{C} 23$ & $\mathrm{C} 25$ & $1.526(6)$ \\
\hline $\mathrm{Si} 2$ & $\mathrm{C} 43$ & $1.870(5)$ & $\mathrm{C} 26$ & $\mathrm{C} 27$ & $1.396(5)$ \\
\hline $\mathrm{Si} 3$ & C37 & $1.816(4)$ & $\mathrm{C} 26$ & C31 & $1.392(5)$ \\
\hline $\mathrm{Si} 3$ & $\mathrm{C} 38$ & $1.872(5)$ & $\mathrm{C} 27$ & $\mathrm{C} 28$ & $1.400(5)$ \\
\hline $\mathrm{Si} 3$ & C39 & $1.880(4)$ & $\mathrm{C} 28$ & $\mathrm{C} 29$ & $1.387(6)$ \\
\hline $\mathrm{Si} 3$ & $\mathrm{C} 40$ & $1.894(5)$ & $\mathrm{C} 29$ & $\mathrm{C} 30$ & $1.405(6)$ \\
\hline N1 & $\mathrm{C} 1$ & $1.374(4)$ & $\mathrm{C} 29$ & $\mathrm{C} 32$ & $1.515(5)$ \\
\hline N1 & $\mathrm{C} 4$ & $1.374(4)$ & C30 & C31 & $1.385(5)$ \\
\hline $\mathrm{N} 2$ & N3 & $1.350(4)$ & $\mathrm{C} 32$ & $\mathrm{C} 33$ & $1.511(7)$ \\
\hline N3 & N4 & $1.276(4)$ & $\mathrm{C} 32$ & $\mathrm{C} 34$ & $1.512(6)$ \\
\hline N4 & $\mathrm{C} 26$ & $1.418(5)$ & & & \\
\hline
\end{tabular}


Table S5. Bond Angles for 4.

\begin{tabular}{|c|c|c|c|c|c|c|c|}
\hline Atom & Atom & Atom & Angle $/^{\circ}$ & Atom & Atom & Atom & Angle $/^{\circ}$ \\
\hline N1 & Th1 & N4 & $106.60(8)$ & N3 & $\mathrm{N} 2$ & Th1 & $109.4(2)$ \\
\hline N1 & Th1 & N7 & $105.00(8)$ & N3 & $\mathrm{N} 2$ & $\mathrm{P} 1$ & 115.9(2) \\
\hline $\mathrm{N} 2$ & Th1 & N1 & $61.84(9)$ & N4 & $\mathrm{N} 3$ & $\mathrm{~N} 2$ & $108.9(3)$ \\
\hline $\mathrm{N} 2$ & Th1 & N4 & $45.61(9)$ & N3 & N4 & Th1 & $95.78(19)$ \\
\hline $\mathrm{N} 2$ & Th1 & N7 & $153.52(9)$ & $\mathrm{N} 3$ & $\mathrm{~N} 4$ & $\mathrm{C} 26$ & $113.0(3)$ \\
\hline $\mathrm{N} 2$ & Th1 & C37 & $80.63(11)$ & $\mathrm{C} 26$ & N4 & Th1 & $150.6(2)$ \\
\hline $\mathrm{N} 4$ & Th1 & N7 & $144.78(8)$ & $\mathrm{P} 2$ & N5 & Th1 & $131.76(16)$ \\
\hline N5 & Th1 & N1 & $62.36(9)$ & N6 & N5 & Th1 & $109.2(2)$ \\
\hline N5 & Th1 & N2 & 123.71(9) & N6 & N5 & $\mathrm{P} 2$ & 113.1(2) \\
\hline N5 & Th1 & $\mathrm{N} 4$ & $168.90(9)$ & N7 & N6 & N5 & 109.4(3) \\
\hline N5 & Th1 & N7 & $45.71(9)$ & N6 & N7 & Th1 & $94.4(2)$ \\
\hline N5 & Th1 & C37 & $104.47(11)$ & N6 & N7 & C17 & 114.1(3) \\
\hline C35 & Th1 & N1 & $173.66(10)$ & C17 & N7 & Th1 & $149.5(2)$ \\
\hline C35 & Th1 & N2 & $122.44(10)$ & N1 & $\mathrm{C} 1$ & $\mathrm{P} 1$ & $116.5(3)$ \\
\hline C35 & Th1 & $\mathrm{N} 4$ & $77.08(10)$ & N1 & $\mathrm{C} 1$ & $\mathrm{C} 2$ & 111.9(3) \\
\hline C35 & Th1 & N5 & $113.79(11)$ & $\mathrm{C} 2$ & $\mathrm{C} 1$ & $\mathrm{P} 1$ & $131.5(3)$ \\
\hline C35 & Th1 & N7 & $73.02(10)$ & $\mathrm{C} 1$ & $\mathrm{C} 2$ & C3 & $106.0(3)$ \\
\hline C35 & Th1 & C37 & $88.13(11)$ & $\mathrm{C} 4$ & $\mathrm{C} 3$ & $\mathrm{C} 2$ & 106.2(3) \\
\hline $\mathrm{C} 36$ & Th1 & N1 & $91.64(10)$ & N1 & $\mathrm{C} 4$ & $\mathrm{P} 2$ & $115.7(2)$ \\
\hline C36 & Th1 & $\mathrm{N} 2$ & $79.45(11)$ & $\mathrm{N} 1$ & $\mathrm{C} 4$ & $\mathrm{C} 3$ & 111.9(3) \\
\hline C36 & Th1 & N4 & $85.88(10)$ & $\mathrm{C} 3$ & $\mathrm{C} 4$ & $\mathrm{P} 2$ & $131.8(3)$ \\
\hline C36 & Th1 & N5 & $95.24(11)$ & C6 & $\mathrm{C} 5$ & $\mathrm{P} 1$ & $110.4(3)$ \\
\hline $\mathrm{C} 36$ & Th1 & N7 & $78.04(10)$ & C6 & $\mathrm{C} 5$ & $\mathrm{C} 7$ & 111.7(4) \\
\hline $\mathrm{C} 36$ & Th1 & $\mathrm{C} 35$ & $93.80(12)$ & $\mathrm{C} 7$ & $\mathrm{C} 5$ & $\mathrm{P} 1$ & $111.0(3)$ \\
\hline C36 & Th1 & C37 & $157.51(12)$ & C9 & $\mathrm{C} 8$ & $\mathrm{P} 1$ & $110.1(3)$ \\
\hline C37 & Th1 & N1 & $88.10(10)$ & C9 & $\mathrm{C} 8$ & C10 & $111.0(3)$ \\
\hline C37 & Th1 & N4 & $72.70(10)$ & $\mathrm{C} 10$ & $\mathrm{C} 8$ & $\mathrm{P} 1$ & $112.9(3)$ \\
\hline C37 & Th1 & N7 & $123.68(10)$ & C12 & $\mathrm{C} 11$ & $\mathrm{P} 2$ & 108.7(3) \\
\hline $\mathrm{N} 2$ & $\mathrm{P} 1$ & $\mathrm{C} 1$ & $100.72(15)$ & C12 & $\mathrm{C} 11$ & C13 & $110.5(3)$ \\
\hline $\mathrm{N} 2$ & $\mathrm{P} 1$ & $\mathrm{C} 5$ & $113.29(17)$ & C13 & $\mathrm{C} 11$ & $\mathrm{P} 2$ & 113.2(3) \\
\hline $\mathrm{N} 2$ & $\mathrm{P} 1$ & $\mathrm{C} 8$ & $112.08(16)$ & C15 & $\mathrm{C} 14$ & $\mathrm{P} 2$ & 111.1(3) \\
\hline $\mathrm{C} 1$ & $\mathrm{P} 1$ & $\mathrm{C} 5$ & $108.57(18)$ & C16 & $\mathrm{C} 14$ & $\mathrm{P} 2$ & $114.2(3)$ \\
\hline $\mathrm{C} 1$ & $\mathrm{P} 1$ & $\mathrm{C} 8$ & $113.16(17)$ & C16 & C14 & C15 & $113.3(3)$ \\
\hline $\mathrm{C} 8$ & $\mathrm{P} 1$ & $\mathrm{C} 5$ & $108.86(18)$ & C18 & C17 & N7 & $124.0(3)$ \\
\hline N5 & $\mathrm{P} 2$ & $\mathrm{C} 4$ & $101.37(16)$ & C18 & $\mathrm{C} 17$ & $\mathrm{C} 22$ & 119.1(4) \\
\hline N5 & $\mathrm{P} 2$ & $\mathrm{C} 11$ & $109.70(16)$ & C22 & $\mathrm{C} 17$ & N7 & $116.9(4)$ \\
\hline N5 & $\mathrm{P} 2$ & $\mathrm{C} 14$ & $110.85(17)$ & C17 & $\mathrm{C} 18$ & C19 & $119.8(4)$ \\
\hline $\mathrm{C} 4$ & $\mathrm{P} 2$ & $\mathrm{C} 11$ & $109.46(17)$ & C18 & C19 & $\mathrm{C} 20$ & $121.5(4)$ \\
\hline $\mathrm{C} 4$ & $\mathrm{P} 2$ & $\mathrm{C} 14$ & $111.89(17)$ & C19 & $\mathrm{C} 20$ & $\mathrm{C} 23$ & $121.3(4)$ \\
\hline $\mathrm{C} 14$ & $\mathrm{P} 2$ & $\mathrm{C} 11$ & $112.96(18)$ & C21 & $\mathrm{C} 20$ & C19 & $117.7(4)$ \\
\hline C35 & Si1 & $\mathrm{C} 44$ & $114.02(19)$ & C21 & $\mathrm{C} 20$ & $\mathrm{C} 23$ & $121.0(4)$ \\
\hline
\end{tabular}




\begin{tabular}{|c|c|c|c|c|c|c|c|c|}
\hline C35 & Si1 & C45 & $113.71(19)$ & & C20 & C21 & C22 & $121.6(4)$ \\
\hline C35 & Si1 & C46 & $108.69(19)$ & & C21 & C22 & C17 & $120.3(4)$ \\
\hline C45 & Si1 & C44 & $103.6(2)$ & & C24 & C23 & C20 & $111.3(3)$ \\
\hline C45 & Si1 & C46 & $108.6(2)$ & & C25 & C23 & C20 & $111.8(4)$ \\
\hline C46 & Si1 & C44 & $107.9(2)$ & & C25 & C23 & C24 & $111.6(4)$ \\
\hline C36 & Si2 & C41 & $112.82(19)$ & & C27 & C26 & N4 & $117.9(3)$ \\
\hline C36 & Si2 & C42 & $113.4(2)$ & & C31 & C26 & N4 & $123.0(3)$ \\
\hline C36 & Si2 & C43 & $108.6(2)$ & & C31 & C26 & C27 & $119.1(3)$ \\
\hline C41 & Si2 & C42 & $106.8(2)$ & & C26 & C27 & C28 & $119.7(4)$ \\
\hline C41 & Si2 & C43 & $109.3(3)$ & & C29 & C28 & C27 & $121.9(4)$ \\
\hline C43 & Si2 & C42 & $105.6(3)$ & & C28 & C29 & C30 & $117.3(4)$ \\
\hline C37 & Si3 & C38 & $108.6(2)$ & & C28 & C29 & C32 & $120.5(4)$ \\
\hline C37 & Si3 & C39 & $114.57(18)$ & & C30 & C29 & C32 & $122.2(4)$ \\
\hline C37 & Si3 & C40 & $115.49(19)$ & & C31 & C30 & C29 & $121.6(4)$ \\
\hline C38 & Si3 & C39 & $108.2(2)$ & & C30 & C31 & C26 & $120.3(4)$ \\
\hline C38 & Si3 & C40 & $104.6(3)$ & & C33 & C32 & C29 & $110.6(4)$ \\
\hline C39 & Si3 & C40 & $104.7(2)$ & & C33 & C32 & C34 & $111.4(5)$ \\
\hline C1 & N1 & Th1 & $128.0(2)$ & & C34 & C32 & C29 & $112.8(4)$ \\
\hline C1 & N1 & C4 & $104.1(3)$ & & Si1 & C35 & Th1 & $131.96(18)$ \\
\hline C4 & N1 & Th1 & $127.7(2)$ & & Si2 & C36 & Th1 & $138.7(2)$ \\
\hline P1 & N2 & Th1 & $132.49(15)$ & & Si3 & C37 & Th1 & $126.51(19$ \\
\hline
\end{tabular}

Table S6. Hydrogen Atom Coordinates $\left(\AA \times 10^{4}\right)$ and Isotropic Displacement Parameters $\left(\AA^{2} \times 10^{3}\right)$ for 4 .

\begin{tabular}{|c|c|c|c|c|}
\hline Atom & $\mathbf{x}$ & $\mathbf{y}$ & $\mathbf{z}$ & $\mathbf{U}(\mathbf{e q})$ \\
\hline H2 & 3191.17 & 5425.79 & 3616.34 & 33 \\
\hline H3 & 3720.91 & 4571.56 & 3288.01 & 32 \\
\hline H5 & 3064.02 & 6811.85 & 4658.49 & 36 \\
\hline H6A & 3680.95 & 6354.79 & 5486.04 & 57 \\
\hline H6B & 3585.08 & 7546.11 & 5208.25 & 57 \\
\hline H6C & 3689 & 6533.93 & 4851.25 & 57 \\
\hline H7A & 2713.43 & 6584.57 & 5319.78 & 55 \\
\hline H7B & 3007.64 & 7511.1 & 5539.47 & 55 \\
\hline H7C & 3054.83 & 6270.42 & 5777 & 55 \\
\hline H8 & 2549.53 & 4594.48 & 4961.02 & 33 \\
\hline H9A & 2705.96 & 3344.64 & 4061.74 & 53 \\
\hline H9B & 2371.95 & 3156.45 & 4353.05 & 53 \\
\hline H9C & 2766.93 & 2875.72 & 4674.82 & 53 \\
\hline H10A & 2488.98 & 6140.88 & 4364.12 & 58 \\
\hline H10B & 2209.59 & 5186.88 & 4123.83 & 58 \\
\hline H10C & 2563.08 & 5359.19 & 3877.4 & 58 \\
\hline H11 & 4849.68 & 3869.61 & 4342.25 & 31 \\
\hline H12A & 4331.51 & 5495.68 & 4507.45 & 50 \\
\hline H12B & 4753.86 & 5694.4 & 4648.23 & 50 \\
\hline
\end{tabular}




\begin{tabular}{|c|c|c|c|c|}
\hline $\mathrm{H} 12 \mathrm{C}$ & 4575.19 & 4792.71 & 4984.8 & 50 \\
\hline H13A & 4672.54 & 4194.36 & 3394.86 & 49 \\
\hline H13B & 4808.95 & 5340.73 & 3690.94 & 49 \\
\hline H13C & 4390.17 & 5111.66 & 3503.05 & 49 \\
\hline H14 & 4130.27 & 1487.16 & 3767.67 & 33 \\
\hline H15A & 4816.26 & 2183.75 & 3608.77 & 57 \\
\hline H15B & 4654.01 & 955.12 & 3512.73 & 57 \\
\hline $\mathrm{H} 15 \mathrm{C}$ & 4753.94 & 1452.86 & 4119.74 & 57 \\
\hline H16A & 3865.9 & 2650.88 & 3062.77 & 53 \\
\hline H16B & 4132.63 & 1822.87 & 2837.62 & 53 \\
\hline $\mathrm{H} 16 \mathrm{C}$ & 4247.2 & 3087.85 & 2976.89 & 53 \\
\hline H18 & 5252.8 & 1777.89 & 5322.24 & 45 \\
\hline H19 & 5802.21 & 1627.22 & 5919.33 & 49 \\
\hline $\mathrm{H} 21$ & 5326.92 & 2136.69 & 7208.13 & 36 \\
\hline $\mathrm{H} 22$ & 4780.36 & 2360.27 & 6617.41 & 35 \\
\hline $\mathrm{H} 23$ & 5936.98 & 1848.31 & 7403.17 & 40 \\
\hline $\mathrm{H} 24 \mathrm{~A}$ & 6275.59 & 2721.48 & 6566.51 & 85 \\
\hline H24B & 6454.99 & 2678.49 & 7203.18 & 85 \\
\hline $\mathrm{H} 24 \mathrm{C}$ & 6114.84 & 3455.36 & 6997.89 & 85 \\
\hline $\mathrm{H} 25 \mathrm{~A}$ & 5994.47 & 63.59 & 7033.9 & 64 \\
\hline $\mathrm{H} 25 \mathrm{~B}$ & 6378.27 & 606.15 & 7254.17 & 64 \\
\hline $\mathrm{H} 25 \mathrm{C}$ & 6216.48 & 583 & 6610.93 & 64 \\
\hline $\mathrm{H} 27$ & 3286.54 & 1267.63 & 6542.12 & 30 \\
\hline $\mathrm{H} 28$ & 2958.62 & 684.07 & 7193.17 & 34 \\
\hline H30 & 2570.87 & 3796.41 & 7264.46 & 39 \\
\hline H31 & 2889.12 & 4385.45 & 6610.76 & 34 \\
\hline $\mathrm{H} 32$ & 2620.3 & 1032.9 & 7859.48 & 43 \\
\hline H33A & 2020.46 & 2242.79 & 7294.77 & 103 \\
\hline H33B & 2002.06 & 1234.14 & 7708.61 & 103 \\
\hline $\mathrm{H} 33 \mathrm{C}$ & 2126.94 & 1011.47 & 7140.15 & 103 \\
\hline H34A & 2788.17 & 2682.49 & 8359.08 & 77 \\
\hline H34B & 2419.28 & 2194.98 & 8472.81 & 77 \\
\hline $\mathrm{H} 34 \mathrm{C}$ & 2418.13 & 3242.25 & 8074.86 & 77 \\
\hline $\mathrm{H} 35 \mathrm{~A}$ & $3857(9)$ & $1660(40)$ & $6533(16)$ & $33(12)$ \\
\hline H35B & $4232(10)$ & $2050(40)$ & $6480(20)$ & $43(14)$ \\
\hline H36A & $4090(13)$ & $5030(30)$ & $5663(16)$ & $45(14)$ \\
\hline H36B & $4400(8)$ & $4200(50)$ & $5960(20)$ & $58(17)$ \\
\hline H37A & $3405(14)$ & $940(40)$ & $5440(14)$ & $50(15)$ \\
\hline H37B & $3139(8)$ & $1810(30)$ & $5150(18)$ & $27(11)$ \\
\hline H38A & 3925.8 & 199.07 & 4511.34 & 73 \\
\hline H38B & 3688.7 & -822.92 & 4233.72 & 73 \\
\hline $\mathrm{H} 38 \mathrm{C}$ & 3773.61 & -691.46 & 4886.07 & 73 \\
\hline H39A & 2996.92 & 1697.12 & 3769.19 & 50 \\
\hline H39B & 3273.86 & 909.99 & 3542.5 & 50 \\
\hline
\end{tabular}




\begin{tabular}{|c|c|c|c|c|}
\hline H39C & 3412.28 & 2008.43 & 3882.15 & 50 \\
\hline H40A & 2937.31 & -892.31 & 4734.67 & 68 \\
\hline H40B & 2961.57 & -996.7 & 4101.76 & 68 \\
\hline H40C & 2704.38 & -87.13 & 4291.74 & 68 \\
\hline H41A & 3621.55 & 6079.4 & 6475.29 & 74 \\
\hline H41B & 3779.56 & 6001.13 & 7118.61 & 74 \\
\hline H41C & 3604.76 & 4926.75 & 6791.94 & 74 \\
\hline H42A & 4710.44 & 6323.41 & 6640.38 & 66 \\
\hline H42B & 4471.55 & 6853.51 & 7037.15 & 66 \\
\hline H42C & 4361.33 & 7027.8 & 6388.73 & 66 \\
\hline H43A & 4280.97 & 3590.51 & 7190.89 & 89 \\
\hline H43B & 4455.69 & 4650.57 & 7530.32 & 89 \\
\hline H43C & 4658.32 & 4056.8 & 7102.25 & 89 \\
\hline H44A & 4722.51 & 221.71 & 7055.08 & 61 \\
\hline H44B & 4570.3 & -1020.81 & 7047.85 & 61 \\
\hline H44C & 4395.02 & -51.12 & 7349.22 & 61 \\
\hline H45A & 4294.1 & -162.08 & 5472.78 & 62 \\
\hline H45B & 4446.62 & -1209.5 & 5842.64 & 62 \\
\hline H45C & 4665.74 & -65.86 & 5897.14 & 62 \\
\hline H46A & 3661.86 & -638.31 & 6661.01 & 64 \\
\hline H46B & 3850.37 & -1610.26 & 6380.89 & 64 \\
\hline H46C & 3609.93 & -703.01 & 6007.97 & 64 \\
\hline
\end{tabular}

Table S7. Fractional Atomic Coordinates $\left(\times 10^{4}\right)$ and Equivalent Isotropic Displacement Parameters $\left(\AA^{2} \times 10^{3}\right)$ for 5 . $U_{\text {eq }}$ is defined as $1 / 3$ of of the trace of the orthogonalised $U_{\text {IJ }}$ tensor.

\begin{tabular}{|c|c|c|c|c|}
\hline Atom & $\mathbf{x}$ & $\mathbf{y}$ & $\mathbf{z}$ & $\mathbf{U}(\mathbf{e q})$ \\
\hline Th1 & $5109.6(2)$ & $6490.1(2)$ & $6994.4(2)$ & $13.94(5)$ \\
\hline C11 & $6360.3(10)$ & $7235.3(4)$ & $8102.7(5)$ & $29.7(2)$ \\
\hline C12 & $3668.4(9)$ & $6302.4(4)$ & $7910.5(5)$ & $27.77(19)$ \\
\hline C13 & $3707.5(9)$ & $5733.6(3)$ & $5971.2(5)$ & $24.67(17)$ \\
\hline P1 & $7878.3(8)$ & $5807.0(3)$ & $6872.9(5)$ & $17.06(17)$ \\
\hline P2 & $4932.2(8)$ & $7466.5(3)$ & $5381.6(5)$ & $14.49(16)$ \\
\hline N1 & $6868(3)$ & $5877.3(11)$ & $7340.7(16)$ & $16.9(6)$ \\
\hline N2 & $4359(3)$ & $7228.1(12)$ & $6053.4(16)$ & $17.0(6)$ \\
\hline N3 & $6423(3)$ & $6646.2(11)$ & $6145.1(16)$ & $15.5(5)$ \\
\hline C00Q & $3240(4)$ & $7990.5(15)$ & $6495(2)$ & $24.0(8)$ \\
\hline C1 & $7396(3)$ & $6316.2(13)$ & $6110.2(19)$ & $15.6(6)$ \\
\hline C2 & $6216(3)$ & $7016.8(13)$ & $5518.7(19)$ & $15.4(6)$ \\
\hline C3 & $7022(3)$ & $6923.6(15)$ & $5095(2)$ & $20.6(7)$ \\
\hline C4 & $7776(4)$ & $6468.7(14)$ & $5475(2)$ & $20.5(7)$ \\
\hline C5 & $3922(3)$ & $7396.9(15)$ & $4356(2)$ & $20.4(7)$ \\
\hline C6 & $3594(4)$ & $6776.0(17)$ & $4162(2)$ & $29.4(8)$ \\
\hline C7 & $2780(4)$ & $7767.7(17)$ & $4181(2)$ & $27.1(8)$ \\
\hline C8 & $5443(4)$ & $8199.4(14)$ & $5557(2)$ & $19.5(7)$ \\
\hline
\end{tabular}




\begin{tabular}{|c|c|c|c|c|}
\hline C9 & $5948(4)$ & $8421.4(15)$ & $4909(2)$ & $27.7(9)$ \\
\hline $\mathrm{C} 10$ & $6398(4)$ & $8248.4(15)$ & $6386(2)$ & $23.3(7)$ \\
\hline $\mathrm{C} 11$ & $9452(4)$ & $5972.4(17)$ & $7476(2)$ & $28.0(8)$ \\
\hline C12 & $10372(4)$ & $5850(2)$ & $7028(3)$ & $40.6(11)$ \\
\hline $\mathrm{C} 13$ & $9506(4)$ & $6589.4(18)$ & $7744(3)$ & $32.8(9)$ \\
\hline $\mathrm{C} 14$ & $7853(4)$ & $5110.9(15)$ & $6430(2)$ & $27.3(8)$ \\
\hline $\mathrm{C} 15$ & $8420(5)$ & $4655.9(17)$ & $7056(3)$ & $38.2(10)$ \\
\hline $\mathrm{C} 16$ & $6547(5)$ & $4962.8(17)$ & $5918(3)$ & $35.6(10)$ \\
\hline $\mathrm{C} 17$ & $6948(3)$ & $5548.0(13)$ & 8036.1(19) & $17.1(7)$ \\
\hline $\mathrm{C} 18$ & $7857(4)$ & $5645.3(14)$ & $8764(2)$ & $22.0(7)$ \\
\hline C19 & $7894(4)$ & $5326.6(15)$ & $9424(2)$ & $24.7(8)$ \\
\hline $\mathrm{C} 20$ & $7020(4)$ & $4907.9(14)$ & 9391(2) & $22.2(7)$ \\
\hline $\mathrm{C} 21$ & $6104(4)$ & $4823.2(16)$ & $8670(2)$ & $24.4(8)$ \\
\hline $\mathrm{C} 22$ & $6059(3)$ & $5134.8(14)$ & $7997(2)$ & $20.1(7)$ \\
\hline $\mathrm{C} 23$ & $7077(4)$ & $4570.0(16)$ & 10132(2) & $27.7(8)$ \\
\hline $\mathrm{C} 24$ & $8269(5)$ & $4234(2)$ & 10432(3) & 41.1(11) \\
\hline $\mathrm{C} 25$ & $6909(6)$ & 4943(2) & 10787(3) & $50.6(14)$ \\
\hline $\mathrm{C} 26$ & $3262(3)$ & 7461.3(14) & $6152.8(19)$ & $17.0(7)$ \\
\hline $\mathrm{C} 27$ & 2191(4) & 7134.1(15) & $5945(2)$ & $23.0(7)$ \\
\hline $\mathrm{C} 28$ & $1107(4)$ & $7344.6(15)$ & $6037(2)$ & $22.9(7)$ \\
\hline C29 & $1066(3)$ & $7878.6(15)$ & $6342(2)$ & $20.4(7)$ \\
\hline $\mathrm{C} 30$ & $2149(4)$ & $8196.2(15)$ & $6577(2)$ & $24.4(8)$ \\
\hline C31 & $-135(4)$ & $8110.7(16)$ & $6416(2)$ & $23.7(8)$ \\
\hline C32 & $-20(4)$ & $8208.3(17)$ & $7292(2)$ & $28.0(8)$ \\
\hline C33 & $-510(5)$ & $8652(2)$ & $5943(3)$ & $39.6(11)$ \\
\hline C1S & $-2162(5)$ & $9039(2)$ & $8629(3)$ & $37.8(10)$ \\
\hline C2S & $-1422(7)$ & 9491(2) & $8972(3)$ & $55.8(15)$ \\
\hline $\mathrm{C} 3 \mathrm{~S}$ & $-218(7)$ & $9442(3)$ & $9350(4)$ & $69.6(19)$ \\
\hline $\mathrm{C} 4 \mathrm{~S}$ & $334(6)$ & $8958(4)$ & $9414(3)$ & $62.6(18)$ \\
\hline $\mathrm{C} 5 \mathrm{~S}$ & $-308(7)$ & $8468(3)$ & $9109(3)$ & $63(2)$ \\
\hline C6S & $-1631(6)$ & $8513.4(19)$ & $8687(3)$ & $42.7(13)$ \\
\hline C7S & $-3521(6)$ & $9099(4)$ & $8251(4)$ & $91(3)$ \\
\hline
\end{tabular}

Table S8. Anisotropic Displacement Parameters $\left(\AA^{2} \times 10^{3}\right)$ for 5. The Anisotropic displacement factor exponent takes the form: $-2 \pi^{2}\left[h^{2} a^{* 2} U_{11}+2 h k a * b * U_{12}+\ldots\right]$.

\begin{tabular}{|c|c|c|c|c|c|c|}
\hline Atom & $\mathbf{U}_{\mathbf{1 1}}$ & $\mathbf{U}_{\mathbf{2 2}}$ & $\mathbf{U}_{\mathbf{3 3}}$ & $\mathbf{U}_{\mathbf{2 3}}$ & $\mathbf{U}_{\mathbf{1 3}}$ & $\mathbf{U}_{\mathbf{1 2}}$ \\
\hline $\mathrm{Th} 1$ & $17.72(8)$ & $14.16(6)$ & $11.43(6)$ & $-0.07(3)$ & $6.74(5)$ & $-0.93(4)$ \\
\hline $\mathrm{C} 11$ & $43.3(6)$ & $28.2(4)$ & $19.4(4)$ & $-7.3(3)$ & $12.5(4)$ & $-13.4(4)$ \\
\hline $\mathrm{C} 12$ & $26.8(5)$ & $40.4(5)$ & $20.6(4)$ & $-0.4(4)$ & $13.8(4)$ & $-7.2(4)$ \\
\hline $\mathrm{C} 13$ & $27.8(5)$ & $21.1(4)$ & $22.2(4)$ & $-3.4(3)$ & $3.9(4)$ & $-2.7(3)$ \\
\hline P1 & $18.7(5)$ & $15.5(4)$ & $16.3(4)$ & $4.1(3)$ & $4.8(4)$ & $3.0(3)$ \\
\hline P2 & $16.4(4)$ & $14.3(3)$ & $13.3(3)$ & $1.4(3)$ & $5.5(3)$ & $1.7(3)$ \\
\hline N1 & $21.0(16)$ & $14.9(12)$ & $13.9(12)$ & $2.8(10)$ & $4.6(12)$ & $-1.3(11)$ \\
\hline
\end{tabular}




\begin{tabular}{|c|c|c|c|c|c|c|}
\hline N2 & $16.0(16)$ & $19.6(13)$ & $16.0(13)$ & $1.1(11)$ & $5.9(12)$ & $3.4(11)$ \\
\hline N3 & $19.7(16)$ & $13.1(12)$ & $13.7(12)$ & $0.2(10)$ & $5.5(12)$ & $2.5(11)$ \\
\hline COOQ & $21(2)$ & $24.8(17)$ & $27.3(18)$ & $-7.2(14)$ & $9.4(17)$ & $-3.5(14)$ \\
\hline $\mathrm{C} 1$ & $15.6(18)$ & $15.7(14)$ & $14.3(14)$ & $3.4(12)$ & $3.3(14)$ & $6.1(13)$ \\
\hline $\mathrm{C} 2$ & 19.8(19) & $14.6(14)$ & $11.9(14)$ & $-0.5(11)$ & $5.1(14)$ & $-0.9(12)$ \\
\hline C3 & $24(2)$ & $22.3(16)$ & $19.8(16)$ & $5.7(13)$ & $13.0(16)$ & $6.5(14)$ \\
\hline $\mathrm{C} 4$ & $21(2)$ & $23.6(17)$ & $19.6(17)$ & $3.8(13)$ & $10.3(16)$ & $5.8(13)$ \\
\hline $\mathrm{C} 5$ & $20.2(19)$ & $27.4(17)$ & $13.5(14)$ & $1.6(13)$ & $5.2(14)$ & $-0.5(14)$ \\
\hline C6 & $34(2)$ & $29.3(19)$ & $20.9(17)$ & $-2.1(15)$ & $3.6(17)$ & $-3.2(17)$ \\
\hline $\mathrm{C} 7$ & $23(2)$ & $31.5(19)$ & $23.3(17)$ & $7.7(15)$ & $2.8(16)$ & $3.9(16)$ \\
\hline $\mathrm{C} 8$ & $24(2)$ & $17.3(15)$ & $17.2(15)$ & $1.2(12)$ & $7.2(15)$ & $0.0(13)$ \\
\hline C9 & $40(3)$ & $20.2(16)$ & $25.7(18)$ & $4.9(14)$ & 14.1(19) & $-6.6(16)$ \\
\hline $\mathrm{C} 10$ & $30(2)$ & $19.9(16)$ & $21.8(17)$ & $-2.6(13)$ & $10.3(16)$ & $-4.3(14)$ \\
\hline $\mathrm{C} 11$ & $24(2)$ & $29.8(19)$ & $26.3(18)$ & $15.2(15)$ & $2.2(17)$ & $4.0(16)$ \\
\hline $\mathrm{C} 12$ & $22(2)$ & $50(3)$ & $50(3)$ & $16(2)$ & $11(2)$ & $8(2)$ \\
\hline $\mathrm{C} 13$ & $24(2)$ & $31.2(19)$ & $34(2)$ & $7.3(17)$ & $-3.5(19)$ & $-11.1(17)$ \\
\hline C14 & $38(2)$ & $18.1(16)$ & $30.4(19)$ & $-0.9(14)$ & 17.7(19) & $4.7(15)$ \\
\hline $\mathrm{C} 15$ & $53(3)$ & $20.6(18)$ & $46(2)$ & $6.5(17)$ & $22(2)$ & $10.5(18)$ \\
\hline $\mathrm{C} 16$ & $50(3)$ & $23.1(18)$ & $34(2)$ & $-11.6(16)$ & $14(2)$ & $0.5(18)$ \\
\hline C17 & $24(2)$ & $12.3(14)$ & $14.8(14)$ & $3.1(12)$ & $6.3(14)$ & $3.2(13)$ \\
\hline $\mathrm{C} 18$ & $29(2)$ & $17.5(15)$ & $16.7(15)$ & $2.9(13)$ & $3.7(16)$ & $-5.5(14)$ \\
\hline C19 & $33(2)$ & $23.6(17)$ & $14.6(15)$ & $3.4(13)$ & $3.3(16)$ & $-4.9(16)$ \\
\hline $\mathrm{C} 20$ & $28(2)$ & $19.2(15)$ & $18.9(16)$ & $3.1(13)$ & $6.7(16)$ & $-4.2(14)$ \\
\hline $\mathrm{C} 21$ & $26(2)$ & $25.8(17)$ & $21.0(17)$ & $-0.4(14)$ & $7.4(16)$ & $-11.6(15)$ \\
\hline $\mathrm{C} 22$ & $18.5(19)$ & $20.7(15)$ & $17.5(15)$ & $1.0(13)$ & $0.7(15)$ & $-3.5(14)$ \\
\hline $\mathrm{C} 23$ & $38(2)$ & $22.5(17)$ & $19.7(17)$ & $8.0(14)$ & $5.9(17)$ & $-7.0(16)$ \\
\hline $\mathrm{C} 24$ & $45(3)$ & $36(2)$ & $32(2)$ & $19.0(19)$ & $-1(2)$ & $-3(2)$ \\
\hline $\mathrm{C} 25$ & $91(4)$ & $39(2)$ & $33(2)$ & $6.4(19)$ & $35(3)$ & $-9(3)$ \\
\hline $\mathrm{C} 26$ & $17.6(18)$ & $18.2(15)$ & $16.8(15)$ & $2.7(12)$ & $7.9(14)$ & $4.1(13)$ \\
\hline $\mathrm{C} 27$ & $23(2)$ & $19.8(16)$ & $30.2(18)$ & $-3.8(14)$ & $13.5(17)$ & $-2.1(14)$ \\
\hline $\mathrm{C} 28$ & $20(2)$ & $22.5(16)$ & $28.5(18)$ & $-0.3(14)$ & $11.1(16)$ & $-2.9(14)$ \\
\hline $\mathrm{C} 29$ & 17.7(19) & $25.9(17)$ & $19.5(16)$ & $5.0(13)$ & $8.6(15)$ & $8.0(14)$ \\
\hline $\mathrm{C} 30$ & $18(2)$ & $24.2(17)$ & $31.9(19)$ & $-7.6(15)$ & $9.7(17)$ & $0.9(14)$ \\
\hline C31 & $19(2)$ & $27.0(17)$ & $28.7(18)$ & $-0.1(15)$ & $12.5(17)$ & $3.7(14)$ \\
\hline $\mathrm{C} 32$ & $26(2)$ & $33.6(19)$ & $28.2(19)$ & $-4.9(16)$ & $14.6(18)$ & $1.7(16)$ \\
\hline C33 & $36(3)$ & $42(2)$ & $48(3)$ & $13(2)$ & $24(2)$ & $13(2)$ \\
\hline $\mathrm{C} 1 \mathrm{~S}$ & $36(3)$ & $45(2)$ & $31(2)$ & $-6.4(19)$ & $9(2)$ & $9(2)$ \\
\hline $\mathrm{C} 2 \mathrm{~S}$ & $81(5)$ & $38(3)$ & $45(3)$ & $7(2)$ & $16(3)$ & $-8(3)$ \\
\hline $\mathrm{C} 3 \mathrm{~S}$ & $63(5)$ & $81(5)$ & $69(4)$ & $6(4)$ & $28(4)$ & $-27(4)$ \\
\hline $\mathrm{C} 4 \mathrm{~S}$ & $35(3)$ & $118(6)$ & $38(3)$ & $3(3)$ & $16(2)$ & $-16(4)$ \\
\hline $\mathrm{C} 5 \mathrm{~S}$ & $91(5)$ & $76(4)$ & $46(3)$ & $37(3)$ & $54(4)$ & $60(4)$ \\
\hline C6S & $68(4)$ & $39(2)$ & $36(2)$ & $-7.5(18)$ & $37(3)$ & $-12(2)$ \\
\hline $\mathrm{C} 7 \mathrm{~S}$ & $43(4)$ & $168(8)$ & $51(3)$ & $-31(5)$ & $1(3)$ & $44(5)$ \\
\hline
\end{tabular}


Table S9. Bond Lengths for 5.

\begin{tabular}{|c|c|c|c|c|c|c|}
\hline Atom & Atom & Length/A & & Atom & Atom & Length/A \\
\hline Th1 & C11 & $2.6882(9)$ & & C11 & C12 & $1.535(6)$ \\
\hline Th1 & C12 & $2.6983(8)$ & & C11 & C13 & $1.533(6)$ \\
\hline Th1 & C13 & $2.6859(9)$ & & C14 & C15 & $1.534(6)$ \\
\hline Th1 & N1 & $2.397(3)$ & & C14 & C16 & $1.520(7)$ \\
\hline Th1 & N2 & $2.380(3)$ & & C17 & C18 & $1.393(5)$ \\
\hline Th1 & N3 & $2.474(3)$ & & C17 & C22 & $1.399(5)$ \\
\hline P1 & N1 & $1.634(3)$ & & C18 & C19 & $1.383(5)$ \\
\hline P1 & C1 & $1.764(3)$ & & C19 & C20 & $1.397(5)$ \\
\hline P1 & C11 & $1.819(4)$ & & C20 & C21 & $1.385(5)$ \\
\hline P1 & C14 & $1.824(4)$ & & C20 & C23 & $1.522(5)$ \\
\hline P2 & N2 & $1.634(3)$ & & C21 & C22 & $1.390(5)$ \\
\hline P2 & C2 & $1.769(3)$ & & C23 & C24 & $1.521(7)$ \\
\hline P2 & C5 & $1.823(4)$ & & C23 & C25 & $1.521(6)$ \\
\hline P2 & C8 & $1.827(3)$ & & C26 & C27 & $1.397(5)$ \\
\hline N1 & C17 & $1.437(4)$ & & C27 & C28 & $1.395(5)$ \\
\hline N2 & C26 & $1.434(4)$ & & C28 & C29 & $1.383(5)$ \\
\hline N3 & C1 & $1.379(4)$ & & C29 & C30 & $1.395(5)$ \\
\hline N3 & C2 & $1.376(4)$ & & C29 & C31 & $1.525(5)$ \\
\hline C00Q & C26 & $1.397(5)$ & & C31 & C32 & $1.533(5)$ \\
\hline C00Q & C30 & $1.392(5)$ & & C31 & C33 & $1.519(6)$ \\
\hline C1 & C4 & $1.380(4)$ & & C1S & C2S & $1.380(8)$ \\
\hline C2 & C3 & $1.381(4)$ & & C1S & C6S & $1.377(7)$ \\
\hline C3 & C4 & $1.410(5)$ & & C1S & C7S & $1.490(8)$ \\
\hline C5 & C6 & $1.530(5)$ & & C2S & C3S & $1.331(10)$ \\
\hline C5 & C7 & $1.523(5)$ & & C3S & C4S & $1.296(10)$ \\
\hline C8 & C9 & $1.536(5)$ & & C4S & C5S & $1.390(10)$ \\
\hline C8 & C10 & $1.529(5)$ & & C5S & C6S & $1.462(9)$ \\
\hline
\end{tabular}

Table S10. Bond Angles for 5.

\begin{tabular}{|c|c|c|c|l|c|c|c|c|}
\hline Atom & Atom & Atom & Angle $^{\circ}$ & & Atom & Atom & Atom & Angle $^{\circ}$ \\
\hline C11 & Th1 & C12 & $87.33(3)$ & & C6 & C5 & P2 & $110.0(3)$ \\
\hline C13 & Th1 & C11 & $174.34(3)$ & & C7 & C5 & P2 & $112.3(2)$ \\
\hline C13 & Th1 & C12 & $87.03(3)$ & & C7 & C5 & C6 & $112.1(3)$ \\
\hline N1 & Th1 & C11 & $90.25(7)$ & & C9 & C8 & P2 & $112.0(2)$ \\
\hline N1 & Th1 & C12 & $111.81(7)$ & & C10 & C8 & P2 & $109.1(2)$ \\
\hline N1 & Th1 & C13 & $92.25(7)$ & & C10 & C8 & C9 & $111.6(3)$ \\
\hline N1 & Th1 & N3 & $67.79(9)$ & & C12 & C11 & P1 & $111.7(3)$ \\
\hline N2 & Th1 & Cl1 & $90.99(7)$ & & C13 & C11 & P1 & $108.8(3)$ \\
\hline N2 & Th1 & Cl2 & $112.98(7)$ & & C13 & C11 & C12 & $112.0(4)$ \\
\hline N2 & Th1 & Cl3 & $90.81(7)$ & & C15 & C14 & P1 & $112.3(3)$ \\
\hline
\end{tabular}




\begin{tabular}{|c|c|c|c|c|c|c|c|}
\hline $\mathrm{N} 2$ & Th1 & N1 & \begin{tabular}{|l|}
$135.20(9)$ \\
\end{tabular} & C16 & C14 & $\mathrm{P} 1$ & $110.0(3)$ \\
\hline $\mathrm{N} 2$ & Th1 & N3 & $67.44(9)$ & C16 & $\mathrm{C} 14$ & $\mathrm{C} 15$ & $112.1(4)$ \\
\hline N3 & Th1 & $\mathrm{Cl1}$ & $93.53(7)$ & C18 & C17 & $\mathrm{N} 1$ & $121.9(3)$ \\
\hline N3 & Th1 & $\mathrm{Cl} 2$ & \begin{tabular}{|l|}
$179.05(6)$ \\
\end{tabular} & C18 & C17 & $\mathrm{C} 22$ & $118.5(3)$ \\
\hline N3 & Th1 & $\mathrm{Cl} 3$ & $92.12(7)$ & $\mathrm{C} 22$ & $\mathrm{C} 17$ & $\mathrm{~N} 1$ & $119.5(3)$ \\
\hline $\mathrm{N} 1$ & $\mathrm{P} 1$ & $\mathrm{C} 1$ & $102.78(15)$ & C19 & C18 & $\mathrm{C} 17$ & $120.3(3)$ \\
\hline N1 & $\mathrm{P} 1$ & C11 & $114.16(17)$ & C18 & C19 & $\mathrm{C} 20$ & $121.7(4)$ \\
\hline $\mathrm{N} 1$ & $\mathrm{P} 1$ & C14 & $113.26(16)$ & C19 & $\mathrm{C} 20$ & $\mathrm{C} 23$ & $120.4(3)$ \\
\hline $\mathrm{C} 1$ & $\mathrm{P} 1$ & C11 & $108.08(17)$ & $\mathrm{C} 21$ & C20 & C19 & $117.5(3)$ \\
\hline $\mathrm{C} 1$ & $\mathrm{P} 1$ & C14 & $109.32(17)$ & $\mathrm{C} 21$ & $\mathrm{C} 20$ & $\mathrm{C} 23$ & $122.1(3)$ \\
\hline $\mathrm{C} 11$ & $\mathrm{P} 1$ & $\mathrm{C} 14$ & $108.9(2)$ & $\mathrm{C} 20$ & $\mathrm{C} 21$ & $\mathrm{C} 22$ & $121.6(3)$ \\
\hline $\mathrm{N} 2$ & $\mathrm{P} 2$ & $\mathrm{C} 2$ & $101.64(15)$ & $\mathrm{C} 21$ & $\mathrm{C} 22$ & $\mathrm{C} 17$ & $120.2(3)$ \\
\hline $\mathrm{N} 2$ & $\mathrm{P} 2$ & $\mathrm{C} 5$ & $114.94(16)$ & $\mathrm{C} 24$ & $\mathrm{C} 23$ & $\mathrm{C} 20$ & $111.2(3)$ \\
\hline $\mathrm{N} 2$ & $\mathrm{P} 2$ & $\mathrm{C} 8$ & $112.70(15)$ & $\mathrm{C} 25$ & $\mathrm{C} 23$ & $\mathrm{C} 20$ & $111.9(3)$ \\
\hline $\mathrm{C} 2$ & $\mathrm{P} 2$ & $\mathrm{C} 5$ & $108.40(16)$ & $\mathrm{C} 25$ & $\mathrm{C} 23$ & $\mathrm{C} 24$ & $110.9(4)$ \\
\hline $\mathrm{C} 2$ & $\mathrm{P} 2$ & $\mathrm{C} 8$ & $110.07(17)$ & C00Q & $\mathrm{C} 26$ & $\mathrm{~N} 2$ & $122.6(3)$ \\
\hline $\mathrm{C} 5$ & $\mathrm{P} 2$ & $\mathrm{C} 8$ & $108.77(16)$ & C00Q & $\mathrm{C} 26$ & $\mathrm{C} 27$ & $118.4(3)$ \\
\hline $\mathrm{P} 1$ & N1 & Th1 & $127.22(14)$ & $\mathrm{C} 27$ & $\mathrm{C} 26$ & $\mathrm{~N} 2$ & $118.9(3)$ \\
\hline $\mathrm{C} 17$ & $\mathrm{~N} 1$ & Th1 & $111.5(2)$ & $\mathrm{C} 28$ & $\mathrm{C} 27$ & $\mathrm{C} 26$ & $120.6(3)$ \\
\hline $\mathrm{C} 17$ & N1 & $\mathrm{P} 1$ & $121.3(2)$ & $\mathrm{C} 29$ & $\mathrm{C} 28$ & $\mathrm{C} 27$ & 121.1(3) \\
\hline $\mathrm{P} 2$ & $\mathrm{~N} 2$ & Th1 & $128.78(15)$ & $\mathrm{C} 28$ & $\mathrm{C} 29$ & C30 & $118.2(3)$ \\
\hline $\mathrm{C} 26$ & $\mathrm{~N} 2$ & Th1 & $109.30(19)$ & $\mathrm{C} 28$ & C29 & C31 & $120.6(3)$ \\
\hline $\mathrm{C} 26$ & $\mathrm{~N} 2$ & $\mathrm{P} 2$ & $121.9(2)$ & C30 & $\mathrm{C} 29$ & C31 & $121.3(3)$ \\
\hline $\mathrm{C} 1$ & N3 & Th1 & $126.9(2)$ & CO0Q & C30 & C29 & $121.4(3)$ \\
\hline $\mathrm{C} 2$ & N3 & Th1 & $127.3(2)$ & $\mathrm{C} 29$ & C31 & C32 & $110.8(3)$ \\
\hline $\mathrm{C} 2$ & N3 & $\mathrm{C} 1$ & $105.0(3)$ & C33 & C31 & $\mathrm{C} 29$ & 111.1(3) \\
\hline $\mathrm{C} 30$ & $\mathrm{COOQ}$ & $\mathrm{C} 26$ & 120.1(3) & $\mathrm{C} 33$ & C31 & C32 & $110.5(3)$ \\
\hline N3 & $\mathrm{C} 1$ & $\mathrm{P} 1$ & $114.3(2)$ & $\mathrm{C} 2 \mathrm{~S}$ & $\mathrm{C} 1 \mathrm{~S}$ & C7S & $122.0(6)$ \\
\hline N3 & $\mathrm{C} 1$ & $\mathrm{C} 4$ & $111.0(3)$ & C6S & C1S & $\mathrm{C} 2 \mathrm{~S}$ & $118.4(5)$ \\
\hline $\mathrm{C} 4$ & $\mathrm{C} 1$ & $\mathrm{P} 1$ & $134.6(3)$ & C6S & C1S & C7S & $119.5(6)$ \\
\hline N3 & $\mathrm{C} 2$ & $\mathrm{P} 2$ & $114.6(2)$ & C3S & $\mathrm{C} 2 \mathrm{~S}$ & C1S & $123.0(6)$ \\
\hline N3 & $\mathrm{C} 2$ & $\mathrm{C} 3$ & $111.4(3)$ & $\mathrm{C} 4 \mathrm{~S}$ & C3S & $\mathrm{C} 2 \mathrm{~S}$ & $121.3(7)$ \\
\hline $\mathrm{C} 3$ & $\mathrm{C} 2$ & $\mathrm{P} 2$ & $133.8(3)$ & C3S & $\mathrm{C} 4 \mathrm{~S}$ & C5S & $121.6(6)$ \\
\hline $\mathrm{C} 2$ & $\mathrm{C} 3$ & $\mathrm{C} 4$ & $106.0(3)$ & $\mathrm{C} 4 \mathrm{~S}$ & C5S & C6S & $118.0(5)$ \\
\hline $\mathrm{C} 1$ & $\mathrm{C} 4$ & $\mathrm{C} 3$ & 106.6(3) & $\mathrm{C} 1 \mathrm{~S}$ & C6S & C5S & $117.8(5)$ \\
\hline
\end{tabular}

Table S11. Hydrogen Atom Coordinates $\left(\AA \times 10^{4}\right)$ and Isotropic Displacement Parameters $\left(\AA^{2} \times 10^{3}\right)$ for 5.

\begin{tabular}{|c|c|c|c|c|}
\hline Atom & $\mathbf{x}$ & $\mathbf{y}$ & $\mathbf{z}$ & $\mathbf{U}(\mathbf{e q})$ \\
\hline H00Q & 3971.55 & 8210.33 & 6671.62 & 29 \\
\hline H3 & 7059.62 & 7124.9 & 4640.17 & 25 \\
\hline H4 & 8420.2 & 6300.36 & 5323.37 & 25 \\
\hline
\end{tabular}




\begin{tabular}{|c|c|c|c|c|}
\hline $\mathrm{H} 5$ & 4396.97 & 7526.67 & 4002.8 & 25 \\
\hline H6A & 4352.7 & 6554.73 & 4266.74 & 44 \\
\hline H6B & 3099.85 & 6739.66 & 3599.23 & 44 \\
\hline H6C & 3119.76 & 6636.19 & 4495.08 & 44 \\
\hline H7A & 2260.88 & 7633.04 & 4488.12 & 41 \\
\hline H7B & 2314.7 & 7750.5 & 3611.17 & 41 \\
\hline $\mathrm{H} 7 \mathrm{C}$ & 3029.74 & 8158.26 & 4330.46 & 41 \\
\hline $\mathrm{H} 8$ & 4714.08 & 8435.17 & 5547.99 & 23 \\
\hline H9A & 6632.9 & 8182.14 & 4884.96 & 41 \\
\hline H9B & 6240.5 & 8809.39 & 5035.76 & 41 \\
\hline $\mathrm{H} 9 \mathrm{C}$ & 5292.1 & 8414.28 & 4392.95 & 41 \\
\hline H10A & 6058.63 & 8090.51 & 6781.66 & 35 \\
\hline H10B & 6605.91 & 8646.45 & 6507.49 & 35 \\
\hline $\mathrm{H} 10 \mathrm{C}$ & 7143.48 & 8039.7 & 6398.44 & 35 \\
\hline H11 & 9672.8 & 5728.57 & 7961.21 & 34 \\
\hline H12A & 10380.68 & 5444.37 & 6923.98 & 61 \\
\hline H12B & 11198.06 & 5971.78 & 7353.35 & 61 \\
\hline $\mathrm{H} 12 \mathrm{C}$ & 10123.54 & 6056 & 6522.39 & 61 \\
\hline H13A & 9354.04 & 6837.43 & 7280.12 & 49 \\
\hline H13B & 10323.47 & 6669.75 & 8123.49 & 49 \\
\hline $\mathrm{H} 13 \mathrm{C}$ & 8876.38 & 6655.29 & 7999.58 & 49 \\
\hline H14 & 8367.16 & 5132.17 & 6069.12 & 33 \\
\hline $\mathrm{H} 15 \mathrm{~A}$ & 9265.41 & 4763.59 & 7363.73 & 57 \\
\hline H15B & 8431.16 & 4294.65 & 6789.25 & 57 \\
\hline $\mathrm{H} 15 \mathrm{C}$ & 7927.06 & 4618.94 & 7413.84 & 57 \\
\hline H16A & 6554.79 & 4606.87 & 5638.91 & 53 \\
\hline H16B & 6219.22 & 5263.63 & 5528.59 & 53 \\
\hline $\mathrm{H} 16 \mathrm{C}$ & 6024.58 & 4922.53 & 6257.92 & 53 \\
\hline H18 & 8454.74 & 5932.06 & 8807.39 & 26 \\
\hline H19 & 8529.56 & 5394.14 & 9913.44 & 30 \\
\hline $\mathrm{H} 21$ & 5490.35 & 4545.23 & 8633.46 & 29 \\
\hline $\mathrm{H} 22$ & 5421.18 & 5066.93 & 7508.59 & 24 \\
\hline $\mathrm{H} 23$ & 6378.99 & 4294.12 & 9979.48 & 33 \\
\hline $\mathrm{H} 24 \mathrm{~A}$ & 8330.8 & 3978.76 & 10011.87 & 62 \\
\hline $\mathrm{H} 24 \mathrm{~B}$ & 8270.21 & 4013.11 & 10899.85 & 62 \\
\hline $\mathrm{H} 24 \mathrm{C}$ & 8972.41 & 4493.15 & 10578.12 & 62 \\
\hline $\mathrm{H} 25 \mathrm{~A}$ & 7600.96 & 5207.23 & 10967.69 & 76 \\
\hline $\mathrm{H} 25 \mathrm{~B}$ & 6880.52 & 4707.43 & 11235.53 & 76 \\
\hline $\mathrm{H} 25 \mathrm{C}$ & 6135.9 & 5154.63 & 10581.44 & 76 \\
\hline $\mathrm{H} 27$ & 2200.45 & 6764.93 & 5738.15 & 28 \\
\hline $\mathrm{H} 28$ & 385.56 & 7117.43 & 5888.39 & 28 \\
\hline H30 & 2141.18 & 8560.84 & 6797.7 & 29 \\
\hline H31 & -796.97 & 7824.29 & 6193.16 & 28 \\
\hline H32A & 155.24 & 7849.08 & 7579.83 & 42 \\
\hline
\end{tabular}




\begin{tabular}{|c|c|c|c|c|}
\hline H32B & -794.4 & 8364.08 & 7325.49 & 42 \\
\hline H32C & 653.18 & 8474.23 & 7529.9 & 42 \\
\hline H33A & -1290.69 & 8788.29 & 5991.54 & 59 \\
\hline H33B & -611.85 & 8579.38 & 5381.13 & 59 \\
\hline H33C & 130.64 & 8938.57 & 6149.63 & 59 \\
\hline H2S & -1788.42 & 9853.87 & 8937.57 & 67 \\
\hline H3S & 246.68 & 9767.16 & 9576.98 & 83 \\
\hline H4S & 1198.98 & 8938.75 & 9675.51 & 75 \\
\hline H5S & 99.64 & 8113.11 & 9172.46 & 76 \\
\hline H6S & -2107.25 & 8190.9 & 8461.18 & 51 \\
\hline H7SA & -3692.67 & 9370.1 & 7811.67 & 137 \\
\hline H7SB & -3887.42 & 9233.7 & 8647.12 & 137 \\
\hline H7SC & -3877.69 & 8731.94 & 8045.34 & 137 \\
\hline
\end{tabular}

Table S12. Fractional Atomic Coordinates $\left(\times 10^{4}\right)$ and Equivalent Isotropic Displacement Parameters $\left(\AA^{2} \times 10^{3}\right)$ for Complex 6. $U_{\text {eq }}$ is defined as $1 / 3$ of of the trace of the orthogonalised $U_{\text {IJ }}$ tensor.

\begin{tabular}{|c|c|c|c|c|}
\hline Atom & $\mathbf{x}$ & $\mathbf{y}$ & $\mathbf{z}$ & $\mathbf{U}(\mathbf{e q})$ \\
\hline Th1 & $4733.3(2)$ & $7153.9(2)$ & $3850.8(2)$ & $17.29(5)$ \\
\hline P1 & $4016.1(8)$ & $7814.2(5)$ & $5398.3(5)$ & $19.10(18)$ \\
\hline P2 & $7460.3(7)$ & $6516.4(5)$ & $4609.4(5)$ & $20.48(18)$ \\
\hline Si1 & $2279.7(9)$ & $5932.7(6)$ & $3348.0(6)$ & $26.7(2)$ \\
\hline Si2 & $5761.7(10)$ & $8502.4(6)$ & $2878.3(5)$ & $26.9(2)$ \\
\hline N1 & $3672(3)$ & $7714.0(19)$ & $4635.9(16)$ & $23.2(7)$ \\
\hline N2 & $6695(3)$ & $6658.2(17)$ & $3926.1(16)$ & $21.7(6)$ \\
\hline N3 & $5785(3)$ & $7249.8(17)$ & $4966.0(16)$ & $20.3(6)$ \\
\hline C1 & $5373(3)$ & $7463(2)$ & $5519.9(19)$ & $21.0(7)$ \\
\hline C2 & $6043(3)$ & $7258(2)$ & $6073(2)$ & $24.4(8)$ \\
\hline C3 & $6927(3)$ & $6904(2)$ & $5861(2)$ & $26.8(8)$ \\
\hline C4 & $6744(3)$ & $6909(2)$ & $5193.8(19)$ & $21.3(7)$ \\
\hline C5 & $3261(3)$ & $7307(2)$ & $5925(2)$ & $25.3(8)$ \\
\hline C6 & $3159(4)$ & $6569(2)$ & $5675(2)$ & $32.1(9)$ \\
\hline C7 & $2137(4)$ & $7608(3)$ & $6011(2)$ & $33.5(10)$ \\
\hline C8 & $3977(3)$ & $8696(2)$ & $5679.9(19)$ & $24.2(8)$ \\
\hline C9 & $4508(4)$ & $9172(2)$ & $5230(2)$ & $31.2(9)$ \\
\hline C10 & $4508(4)$ & $8767(2)$ & $6385.9(19)$ & $26.4(8)$ \\
\hline C11 & $8853(3)$ & $6865(3)$ & $4679(2)$ & $30.6(9)$ \\
\hline C12 & $8795(4)$ & $7643(3)$ & $4599(3)$ & $38.2(11)$ \\
\hline C13 & $9569(4)$ & $6658(4)$ & $5307(3)$ & $46.7(13)$ \\
\hline C14 & $7605(4)$ & $5611(2)$ & $4827(2)$ & $31.2(9)$ \\
\hline C15 & $8363(5)$ & $5211(3)$ & $4425(3)$ & $40.3(11)$ \\
\hline C16 & $6476(4)$ & $5278(3)$ & $4790(3)$ & $38.9(11)$ \\
\hline C17 & $7060(3)$ & $6385(2)$ & $3348.4(18)$ & $20.7(7)$ \\
\hline C18 & $6479(3)$ & $5861(2)$ & $3013.1(19)$ & $22.5(7)$ \\
\hline & & & & \\
\hline
\end{tabular}




\begin{tabular}{|l|l|l|c|c|}
\hline C19 & $6777(3)$ & $5612(2)$ & $2432(2)$ & $25.4(8)$ \\
\hline C20 & $7666(3)$ & $5887(2)$ & $2168.6(19)$ & $24.2(8)$ \\
\hline C21 & $8248(4)$ & $6412(2)$ & $2515(2)$ & $29.8(9)$ \\
\hline C22 & $7957(3)$ & $6666(2)$ & $3090(2)$ & $24.3(8)$ \\
\hline C23 & $8002(4)$ & $5635(2)$ & $1537(2)$ & $28.8(9)$ \\
\hline C24 & $8035(5)$ & $6226(3)$ & $1060(3)$ & $42.9(12)$ \\
\hline C25 & $7259(4)$ & $5067(2)$ & $1219(2)$ & $33.6(10)$ \\
\hline C26 & $2835(3)$ & $8010(2)$ & $4176.9(19)$ & $20.8(7)$ \\
\hline C27 & $1872(3)$ & $8345(2)$ & $4303(2)$ & $24.9(8)$ \\
\hline C28 & $1129(3)$ & $8577(2)$ & $3782(2)$ & $23.9(8)$ \\
\hline C29 & $1319(3)$ & $8491(2)$ & $3147(2)$ & $24.2(8)$ \\
\hline C30 & $2298(3)$ & $8162(2)$ & $3040.9(19)$ & $24.0(8)$ \\
\hline C31 & $3067(3)$ & $7910(2)$ & $3541(2)$ & $23.7(8)$ \\
\hline C32 & $481(3)$ & $8729(2)$ & $2582(2)$ & $26.2(8)$ \\
\hline C33 & $995(4)$ & $9159(3)$ & $2092(2)$ & $34.1(10)$ \\
\hline C34 & $-113(4)$ & $8107(3)$ & $2254(2)$ & $35.7(10)$ \\
\hline C35 & $5204(3)$ & $7620(2)$ & $2803(2)$ & $25.6(8)$ \\
\hline C36 & $3724(4)$ & $6053(3)$ & $3732(3)$ & $34.6(10)$ \\
\hline C37 & $7277(4)$ & $8517(3)$ & $2808(2)$ & $36.6(10)$ \\
\hline C38 & $5035(5)$ & $9123(3)$ & $2277(3)$ & $45.0(12)$ \\
\hline C39 & $5642(5)$ & $8853(3)$ & $3710(3)$ & $47.4(14)$ \\
\hline C40 & $2093(5)$ & $6289(3)$ & $2508(3)$ & $48.1(13)$ \\
\hline C41 & $1267(4)$ & $6364(3)$ & $3814(3)$ & $46.0(13)$ \\
\hline C42 & $1878(5)$ & $5004(3)$ & $3277(3)$ & $45.6(13)$ \\
\hline
\end{tabular}

Table S13. Anisotropic Displacement Parameters $\left(\AA^{2} \times 10^{3}\right)$ for Complex 6. The Anisotropic displacement factor exponent takes the form: $-2 \pi^{2}\left[h^{2} a^{* 2} U_{11}+2 h k a * b * U_{12}+\ldots\right]$.

\begin{tabular}{|c|c|c|c|c|c|c|}
\hline Atom & $\mathbf{U}_{\mathbf{1 1}}$ & $\mathbf{U}_{\mathbf{2 2}}$ & $\mathbf{U}_{\mathbf{3 3}}$ & $\mathbf{U}_{\mathbf{2 3}}$ & $\mathbf{U}_{\mathbf{1 3}}$ & $\mathbf{U}_{\mathbf{1 2}}$ \\
\hline Th1 & $16.60(7)$ & $21.34(8)$ & $13.65(7)$ & $-0.28(4)$ & $1.09(5)$ & $-0.25(4)$ \\
\hline P1 & $19.7(4)$ & $23.4(5)$ & $14.3(4)$ & $-0.7(3)$ & $2.8(3)$ & $-1.1(3)$ \\
\hline P2 & $17.6(4)$ & $25.7(5)$ & $17.7(4)$ & $-0.4(4)$ & $1.0(3)$ & $1.8(4)$ \\
\hline Si1 & $22.4(5)$ & $33.9(6)$ & $24.2(5)$ & $-4.4(4)$ & $4.6(4)$ & $-7.4(4)$ \\
\hline Si2 & $31.5(6)$ & $27.5(5)$ & $23.5(5)$ & $1.1(4)$ & $9.9(4)$ & $0.9(5)$ \\
\hline N1 & $21.7(16)$ & $29.4(17)$ & $17.3(16)$ & $2.5(13)$ & $-1.7(12)$ & $-0.4(13)$ \\
\hline N2 & $23.6(16)$ & $25.5(16)$ & $16.5(15)$ & $0.3(12)$ & $4.4(12)$ & $0.4(13)$ \\
\hline N3 & $21.2(15)$ & $23.5(15)$ & $16.9(15)$ & $0.8(12)$ & $5.5(12)$ & $-0.7(12)$ \\
\hline C1 & $18.6(17)$ & $25.9(19)$ & $18.4(18)$ & $0.5(15)$ & $1.8(14)$ & $-0.1(14)$ \\
\hline C2 & $27(2)$ & $31(2)$ & $15.2(18)$ & $-0.8(15)$ & $2.4(15)$ & $0.3(16)$ \\
\hline C3 & $25.0(19)$ & $36(2)$ & $17.7(19)$ & $3.9(16)$ & $-2.5(15)$ & $4.3(17)$ \\
\hline C4 & $20.6(18)$ & $23.4(18)$ & $19.7(18)$ & $-1.4(14)$ & $2.7(14)$ & $-2.5(14)$ \\
\hline C5 & $27(2)$ & $33(2)$ & $16.7(18)$ & $0.9(15)$ & $3.6(15)$ & $-7.2(16)$ \\
\hline C6 & $44(2)$ & $27(2)$ & $27(2)$ & $2.8(17)$ & $10.8(18)$ & $-11.7(18)$ \\
\hline C7 & $28(2)$ & $42(3)$ & $31(2)$ & $1(2)$ & $9.0(18)$ & $-3.7(19)$ \\
\hline
\end{tabular}




\begin{tabular}{|c|c|c|c|c|c|c|}
\hline $\mathrm{C} 8$ & $24.8(18)$ & $26(2)$ & $20.6(19)$ & $0.1(15)$ & $1.0(15)$ & $-1.4(15)$ \\
\hline C9 & $37(2)$ & $29(2)$ & $29(2)$ & $2.6(17)$ & $7.0(18)$ & $-5.6(18)$ \\
\hline $\mathrm{C} 10$ & $32(2)$ & $28(2)$ & 18.7(19) & $-3.8(15)$ & $4.3(16)$ & $-2.3(16)$ \\
\hline $\mathrm{C} 11$ & 19.4(19) & $47(3)$ & $25(2)$ & $-7.6(19)$ & $4.0(16)$ & $-3.1(18)$ \\
\hline $\mathrm{C} 12$ & $37(2)$ & $39(3)$ & $40(3)$ & $-11(2)$ & $11(2)$ & $-14(2)$ \\
\hline $\mathrm{C} 13$ & $25(2)$ & $75(4)$ & $37(3)$ & $-7(3)$ & $-7.0(19)$ & $0(2)$ \\
\hline $\mathrm{C} 14$ & $41(2)$ & $28(2)$ & $24(2)$ & $2.8(17)$ & $3.1(17)$ & $5.3(18)$ \\
\hline $\mathrm{C} 15$ & $47(3)$ & $37(3)$ & $36(3)$ & $-2(2)$ & $3(2)$ & $14(2)$ \\
\hline $\mathrm{C} 16$ & $48(3)$ & $30(2)$ & $39(3)$ & $8(2)$ & $6(2)$ & $-1(2)$ \\
\hline $\mathrm{C} 17$ & $22.1(17)$ & $23.9(18)$ & $16.0(17)$ & $2.2(14)$ & $1.6(13)$ & $4.3(14)$ \\
\hline $\mathrm{C} 18$ & $21.2(18)$ & 27.4(19) & $19.3(18)$ & $1.2(15)$ & $4.2(14)$ & $-0.6(15)$ \\
\hline C19 & $26.2(19)$ & $28(2)$ & 21.1(19) & $-1.8(16)$ & $1.3(15)$ & $-1.6(16)$ \\
\hline $\mathrm{C} 20$ & 26.1(19) & $25.3(19)$ & 21.1(19) & $1.6(15)$ & $3.2(15)$ & $1.7(15)$ \\
\hline $\mathrm{C} 21$ & $29(2)$ & $33(2)$ & $29(2)$ & $-3.2(18)$ & $10.1(17)$ & $-3.1(17)$ \\
\hline $\mathrm{C} 22$ & $22.8(18)$ & $26.1(19)$ & $25(2)$ & $-2.0(15)$ & $7.9(15)$ & $-2.6(15)$ \\
\hline $\mathrm{C} 23$ & $35(2)$ & $29(2)$ & $24(2)$ & $0.7(16)$ & $7.5(17)$ & $3.0(17)$ \\
\hline $\mathrm{C} 24$ & $63(3)$ & $36(3)$ & $33(3)$ & $2(2)$ & $20(2)$ & $0(2)$ \\
\hline $\mathrm{C} 25$ & $41(2)$ & $32(2)$ & $29(2)$ & $-6.1(18)$ & 9.5(19) & $3.7(19)$ \\
\hline $\mathrm{C} 26$ & $14.7(16)$ & $25.9(18)$ & 21.3(19) & $1.8(15)$ & $0.7(14)$ & $2.0(14)$ \\
\hline $\mathrm{C} 27$ & $22.2(18)$ & $33(2)$ & $20.5(19)$ & $-1.3(16)$ & $4.3(15)$ & $1.8(16)$ \\
\hline $\mathrm{C} 28$ & $17.7(17)$ & $30(2)$ & $26(2)$ & $-1.9(16)$ & $6.3(14)$ & $6.0(15)$ \\
\hline $\mathrm{C} 29$ & $23.2(18)$ & $24.3(19)$ & $24.8(19)$ & $2.4(15)$ & $1.7(15)$ & $2.6(15)$ \\
\hline C30 & $23.1(18)$ & $30(2)$ & $18.3(18)$ & $0.3(15)$ & $0.0(14)$ & $3.1(16)$ \\
\hline C31 & $23.6(19)$ & $30(2)$ & $17.9(19)$ & $-0.7(15)$ & $3.5(15)$ & $-1.9(15)$ \\
\hline C32 & $17.5(17)$ & $38(2)$ & $22.3(19)$ & $2.6(16)$ & $-0.2(14)$ & $6.8(16)$ \\
\hline C33 & $30(2)$ & $38(2)$ & $32(2)$ & $9.2(19)$ & $-0.1(18)$ & $6.9(19)$ \\
\hline C34 & $24(2)$ & $52(3)$ & $31(2)$ & $-1(2)$ & $1.5(17)$ & $-2.2(19)$ \\
\hline C35 & $25.5(19)$ & $28(2)$ & $23(2)$ & $0.0(16)$ & $3.0(15)$ & $4.4(16)$ \\
\hline C36 & $22(2)$ & $38(2)$ & $43(3)$ & $-2(2)$ & $-0.6(18)$ & $-0.5(17)$ \\
\hline C37 & $31(2)$ & $43(3)$ & $37(3)$ & $-1(2)$ & $8.5(19)$ & $-1(2)$ \\
\hline C38 & $45(3)$ & $31(2)$ & $60(3)$ & $6(2)$ & $8(2)$ & $9(2)$ \\
\hline C39 & $48(3)$ & $53(3)$ & $45(3)$ & $-21(3)$ & $19(2)$ & $-19(3)$ \\
\hline $\mathrm{C} 40$ & $54(3)$ & $56(3)$ & $33(3)$ & $5(2)$ & $-1(2)$ & $-12(3)$ \\
\hline $\mathrm{C} 41$ & $29(2)$ & $65(4)$ & $47(3)$ & $-18(3)$ & $15(2)$ & $-9(2)$ \\
\hline $\mathrm{C} 42$ & $48(3)$ & $44(3)$ & $45(3)$ & $-4(2)$ & $5(2)$ & $-23(2)$ \\
\hline
\end{tabular}

Table S14. Bond Lengths for Complex 6.

\begin{tabular}{|c|c|c|c|c|c|}
\hline Atom & Atom & Length/Å & Atom & Atom & Length/Å \\
\hline Th1 & N1 & $2.483(4)$ & $\mathrm{C} 2$ & $\mathrm{C} 3$ & $1.407(6)$ \\
\hline Th1 & $\mathrm{N} 2$ & $2.577(3)$ & $\mathrm{C} 3$ & $\mathrm{C} 4$ & $1.379(6)$ \\
\hline Th1 & N3 & $2.508(3)$ & $\mathrm{C} 5$ & C6 & $1.532(6)$ \\
\hline
\end{tabular}




\begin{tabular}{|c|c|c|l|c|c|c|}
\hline Th1 & C26 & $3.018(4)$ & & C5 & C7 & $1.530(6)$ \\
\hline Th1 & C31 & $2.531(4)$ & & C8 & C9 & $1.529(6)$ \\
\hline Th1 & C35 & $2.509(4)$ & & C8 & C10 & $1.533(5)$ \\
\hline Th1 & C36 & $2.477(5)$ & & C11 & C12 & $1.530(7)$ \\
\hline P1 & N1 & $1.601(3)$ & & C11 & C13 & $1.528(7)$ \\
\hline P1 & C1 & $1.784(4)$ & & C14 & C15 & $1.547(7)$ \\
\hline P1 & C5 & $1.824(4)$ & & C14 & C16 & $1.521(7)$ \\
\hline P1 & C8 & $1.823(4)$ & & C17 & C18 & $1.383(6)$ \\
\hline P2 & N2 & $1.619(3)$ & & C17 & C22 & $1.401(5)$ \\
\hline P2 & C4 & $1.772(4)$ & & C18 & C19 & $1.401(6)$ \\
\hline P2 & C11 & $1.824(4)$ & & C19 & C20 & $1.394(6)$ \\
\hline P2 & C14 & $1.829(5)$ & & C20 & C21 & $1.394(6)$ \\
\hline Si1 & C36 & $1.855(5)$ & & C20 & C23 & $1.517(6)$ \\
\hline Si1 & C40 & $1.872(6)$ & & C21 & C22 & $1.389(6)$ \\
\hline Si1 & C41 & $1.879(5)$ & & C23 & C24 & $1.528(7)$ \\
\hline Si1 & C42 & $1.880(5)$ & & C23 & C25 & $1.529(7)$ \\
\hline Si2 & C35 & $1.853(5)$ & & C26 & C27 & $1.407(5)$ \\
\hline Si2 & C37 & $1.882(5)$ & & C26 & C31 & $1.410(6)$ \\
\hline Si2 & C38 & $1.880(6)$ & & C27 & C28 & $1.395(6)$ \\
\hline Si2 & C39 & $1.891(5)$ & & C28 & C29 & $1.387(6)$ \\
\hline N1 & C26 & $1.426(5)$ & & C29 & C30 & $1.405(6)$ \\
\hline N2 & C17 & $1.445(5)$ & & C29 & C32 & $1.525(5)$ \\
\hline N3 & C1 & $1.388(5)$ & & C30 & C31 & $1.397(6)$ \\
\hline N3 & C4 & $1.377(5)$ & & C32 & C33 & $1.527(6)$ \\
\hline C1 & C2 & $1.381(6)$ & & C32 & C34 & $1.530(7)$ \\
\hline
\end{tabular}

Table S15. Bond Angles for Complex 6.

\begin{tabular}{|c|c|c|c|c|c|c|c|c|}
\hline Atom & Atom & Atom & Angle $^{\circ}$ & & Atom & Atom & Atom & Angle $^{\circ}$ \\
\hline N1 & Th1 & N2 & $134.01(11)$ & & N3 & C1 & P1 & $115.8(3)$ \\
\hline N1 & Th1 & N3 & $66.63(11)$ & & C2 & C1 & P1 & $131.8(3)$ \\
\hline N1 & Th1 & C26 & $27.94(11)$ & & C2 & C1 & N3 & $111.5(3)$ \\
\hline N1 & Th1 & C31 & $55.53(12)$ & & C1 & C2 & C3 & $106.0(3)$ \\
\hline N1 & Th1 & C35 & $128.29(13)$ & & C4 & C3 & C2 & $106.8(4)$ \\
\hline N2 & Th1 & C26 & $159.43(11)$ & & N3 & C4 & P2 & $116.8(3)$ \\
\hline N3 & Th1 & N2 & $67.38(11)$ & & N3 & C4 & C3 & $111.4(3)$ \\
\hline N3 & Th1 & C26 & $93.60(11)$ & & C3 & C4 & P2 & $131.6(3)$ \\
\hline N3 & Th1 & C31 & $119.16(12)$ & & C6 & C5 & P1 & $109.3(3)$ \\
\hline N3 & Th1 & C35 & $129.16(13)$ & & C7 & C5 & P1 & $113.6(3)$ \\
\hline C31 & Th1 & N2 & $161.45(12)$ & & C7 & C5 & C6 & $111.7(4)$ \\
\hline C31 & Th1 & C26 & $27.70(12)$ & & C9 & C8 & P1 & $110.1(3)$ \\
\hline C35 & Th1 & N2 & $82.25(12)$ & & C9 & C8 & C10 & $112.0(4)$ \\
\hline C35 & Th1 & C26 & $105.80(12)$ & & C10 & C8 & P1 & $111.6(3)$ \\
\hline C35 & Th1 & C31 & $80.74(13)$ & & C12 & C11 & P2 & $109.5(3)$ \\
\hline
\end{tabular}




\begin{tabular}{|c|c|c|c|c|c|c|c|}
\hline C36 & Th1 & N1 & $98.58(14)$ & C13 & C11 & $\mathrm{P} 2$ & $112.9(4)$ \\
\hline C36 & Th1 & N2 & $97.36(13)$ & $\mathrm{C} 13$ & $\mathrm{C} 11$ & $\mathrm{C} 12$ & $111.6(4)$ \\
\hline C36 & Th1 & N3 & $110.07(14)$ & $\mathrm{C} 15$ & $\mathrm{C} 14$ & $\mathrm{P} 2$ & $113.6(3)$ \\
\hline C36 & Th1 & $\mathrm{C} 26$ & $96.57(13)$ & $\mathrm{C} 16$ & $\mathrm{C} 14$ & $\mathrm{P} 2$ & $110.2(3)$ \\
\hline C36 & Th1 & C31 & $96.18(14)$ & $\mathrm{C} 16$ & $\mathrm{C} 14$ & $\mathrm{C} 15$ & $111.5(4)$ \\
\hline C36 & Th1 & $\mathrm{C} 35$ & $113.59(16)$ & C18 & $\mathrm{C} 17$ & $\mathrm{~N} 2$ & $120.0(3)$ \\
\hline $\mathrm{N} 1$ & $\mathrm{P} 1$ & $\mathrm{C} 1$ & $101.96(18)$ & $\mathrm{C} 18$ & $\mathrm{C} 17$ & $\mathrm{C} 22$ & $118.2(4)$ \\
\hline $\mathrm{N} 1$ & $\mathrm{P} 1$ & $\mathrm{C5}$ & 116.51(19) & $\mathrm{C} 22$ & $\mathrm{C} 17$ & $\mathrm{~N} 2$ & $121.8(4)$ \\
\hline $\mathrm{N} 1$ & $\mathrm{P} 1$ & $\mathrm{C} 8$ & $114.90(19)$ & $\mathrm{C} 17$ & $\mathrm{C} 18$ & C19 & $121.2(4)$ \\
\hline $\mathrm{C} 1$ & P1 & $\mathrm{C} 5$ & $104.19(19)$ & $\mathrm{C} 20$ & C19 & $\mathrm{C} 18$ & $121.3(4)$ \\
\hline $\mathrm{C} 1$ & $\mathrm{P} 1$ & $\mathrm{C} 8$ & $112.54(19)$ & C19 & $\mathrm{C} 20$ & $\mathrm{C} 23$ & $122.6(4)$ \\
\hline $\mathrm{C} 8$ & $\mathrm{P} 1$ & $\mathrm{C} 5$ & $106.3(2)$ & $\mathrm{C} 21$ & $\mathrm{C} 20$ & C19 & $116.7(4)$ \\
\hline $\mathrm{N} 2$ & $\mathrm{P} 2$ & $\mathrm{C} 4$ & $104.53(18)$ & $\mathrm{C} 21$ & $\mathrm{C} 20$ & $\mathrm{C} 23$ & $120.6(4)$ \\
\hline $\mathrm{N} 2$ & $\mathrm{P} 2$ & C11 & $115.3(2)$ & $\mathrm{C} 22$ & $\mathrm{C} 21$ & $\mathrm{C} 20$ & $122.6(4)$ \\
\hline $\mathrm{N} 2$ & $\mathrm{P} 2$ & $\mathrm{C} 14$ & $114.1(2)$ & $\mathrm{C} 21$ & $\mathrm{C} 22$ & $\mathrm{C} 17$ & $120.0(4)$ \\
\hline $\mathrm{C} 4$ & $\mathrm{P} 2$ & C11 & $109.3(2)$ & $\mathrm{C} 20$ & $\mathrm{C} 23$ & $\mathrm{C} 24$ & $110.9(4)$ \\
\hline $\mathrm{C} 4$ & $\mathrm{P} 2$ & $\mathrm{C} 14$ & $106.7(2)$ & $\mathrm{C} 20$ & $\mathrm{C} 23$ & $\mathrm{C} 25$ & $113.3(4)$ \\
\hline $\mathrm{C} 11$ & $\mathrm{P} 2$ & C14 & $106.5(2)$ & $\mathrm{C} 25$ & $\mathrm{C} 23$ & $\mathrm{C} 24$ & $109.5(4)$ \\
\hline C36 & Si1 & $\mathrm{C} 40$ & $110.2(3)$ & $\mathrm{N} 1$ & $\mathrm{C} 26$ & Th1 & $54.68(19)$ \\
\hline C36 & Si1 & $\mathrm{C} 41$ & $112.2(2)$ & $\mathrm{C} 27$ & $\mathrm{C} 26$ & Th1 & $173.4(3)$ \\
\hline C36 & Si1 & $\mathrm{C} 42$ & $112.3(3)$ & $\mathrm{C} 27$ & C26 & N1 & $127.4(4)$ \\
\hline $\mathrm{C} 40$ & Si1 & $\mathrm{C} 41$ & $108.3(3)$ & $\mathrm{C} 27$ & $\mathrm{C} 26$ & C31 & $121.6(4)$ \\
\hline $\mathrm{C} 40$ & Si1 & $\mathrm{C} 42$ & $106.7(3)$ & C31 & $\mathrm{C} 26$ & Th1 & $56.6(2)$ \\
\hline $\mathrm{C} 41$ & Si1 & $\mathrm{C} 42$ & $106.9(3)$ & C31 & $\mathrm{C} 26$ & N1 & $110.9(3)$ \\
\hline C35 & $\mathrm{Si} 2$ & C37 & $111.3(2)$ & $\mathrm{C} 28$ & $\mathrm{C} 27$ & $\mathrm{C} 26$ & $118.7(4)$ \\
\hline C35 & $\mathrm{Si} 2$ & C38 & $114.1(2)$ & $\mathrm{C} 29$ & $\mathrm{C} 28$ & $\mathrm{C} 27$ & $121.9(4)$ \\
\hline $\mathrm{C} 35$ & $\mathrm{Si} 2$ & C39 & $109.9(2)$ & $\mathrm{C} 28$ & $\mathrm{C} 29$ & $\mathrm{C} 30$ & $117.8(4)$ \\
\hline C37 & $\mathrm{Si} 2$ & C39 & $105.5(2)$ & $\mathrm{C} 28$ & C29 & $\mathrm{C} 32$ & $121.2(4)$ \\
\hline C38 & $\mathrm{Si} 2$ & C37 & $108.6(2)$ & C30 & $\mathrm{C} 29$ & C32 & $121.1(4)$ \\
\hline C38 & $\mathrm{Si} 2$ & C39 & $106.9(3)$ & C31 & C30 & $\mathrm{C} 29$ & $123.2(4)$ \\
\hline $\mathrm{P} 1$ & $\mathrm{~N} 1$ & Th1 & $128.29(19)$ & $\mathrm{C} 26$ & C31 & Th1 & $95.7(3)$ \\
\hline $\mathrm{C} 26$ & $\mathrm{~N} 1$ & Th1 & $97.4(2)$ & C30 & C31 & Th1 & $146.6(3)$ \\
\hline $\mathrm{C} 26$ & $\mathrm{~N} 1$ & P1 & $133.2(3)$ & C30 & C31 & $\mathrm{C} 26$ & $116.9(4)$ \\
\hline $\mathrm{P} 2$ & $\mathrm{~N} 2$ & Th1 & $122.70(17)$ & $\mathrm{C} 29$ & C32 & C33 & $113.0(3)$ \\
\hline $\mathrm{C} 17$ & $\mathrm{~N} 2$ & Th1 & $118.8(2)$ & $\mathrm{C} 29$ & C32 & C34 & $109.4(4)$ \\
\hline $\mathrm{C} 17$ & $\mathrm{~N} 2$ & $\mathrm{P} 2$ & $117.7(3)$ & C33 & C32 & C34 & $110.6(4)$ \\
\hline $\mathrm{C} 1$ & N3 & Th1 & $126.6(3)$ & $\mathrm{Si} 2$ & C35 & Th1 & $112.98(19)$ \\
\hline $\mathrm{C} 4$ & $\mathrm{~N} 3$ & Th1 & $126.1(3)$ & Si1 & C36 & Th1 & $125.9(2)$ \\
\hline $\mathrm{C} 4$ & N3 & $\mathrm{C} 1$ & $104.4(3)$ & & & & \\
\hline
\end{tabular}


Table S16. Hydrogen Atom Coordinates $\left(\AA \times 10^{4}\right)$ and Isotropic Displacement Parameters $\left(\AA^{2} \times 10^{3}\right)$ for Complex 6.

\begin{tabular}{|c|c|c|c|c|}
\hline Atom & $\mathbf{x}$ & $\mathbf{y}$ & $\mathbf{z}$ & $\mathrm{U}(\mathbf{e q})$ \\
\hline $\mathrm{H} 2$ & 5930.75 & 7338.96 & 6507.45 & 29 \\
\hline $\mathrm{H} 3$ & 7533.63 & 6700.59 & 6125.7 & 32 \\
\hline H5 & 3713.92 & 7295.37 & 6361.53 & 30 \\
\hline H6A & 2708.53 & 6561.15 & 5249.37 & 48 \\
\hline H6B & 2810.55 & 6286.74 & 5977.44 & 48 \\
\hline H6C & 3894.36 & 6388.54 & 5638.91 & 48 \\
\hline H7A & 2239.18 & 8062.55 & 6208.47 & 50 \\
\hline H7B & 1770.81 & 7308.03 & 6291.13 & 50 \\
\hline $\mathrm{H} 7 \mathrm{C}$ & 1682.37 & 7646.66 & 5587.92 & 50 \\
\hline $\mathrm{H} 8$ & 3185.22 & 8830.8 & 5656.98 & 29 \\
\hline H9A & 5272.97 & 9030.85 & 5218.9 & 47 \\
\hline H9B & 4493.95 & 9642.53 & 5389.61 & 47 \\
\hline H9C & 4097.94 & 9145.99 & 4793.21 & 47 \\
\hline H10A & 4134.28 & 8461.77 & 6658.6 & 40 \\
\hline H10B & 4438.9 & 9240.93 & 6527.72 & 40 \\
\hline $\mathrm{H} 10 \mathrm{C}$ & 5288.73 & 8643.01 & 6422.6 & 40 \\
\hline H11 & 9206.68 & 6676.29 & 4312.3 & 37 \\
\hline $\mathrm{H} 12 \mathrm{~A}$ & 8308.05 & 7757.52 & 4201.51 & 57 \\
\hline H12B & 9534.64 & 7822.76 & 4575.27 & 57 \\
\hline $\mathrm{H} 12 \mathrm{C}$ & 8505.52 & 7845.66 & 4970.91 & 57 \\
\hline $\mathrm{H} 13 \mathrm{~A}$ & 9265.78 & 6857.83 & 5675.18 & 70 \\
\hline H13B & 10321.49 & 6825.09 & 5302.2 & 70 \\
\hline $\mathrm{H} 13 \mathrm{C}$ & 9577.6 & 6158.16 & 5345.81 & 70 \\
\hline H14 & 7952.36 & 5589.5 & 5288.74 & 37 \\
\hline H15A & 8021.17 & 5191.65 & 3972.44 & 60 \\
\hline H15B & 8470.01 & 4744.81 & 4595.7 & 60 \\
\hline $\mathrm{H} 15 \mathrm{C}$ & 9077.33 & 5441.96 & 4452.41 & 60 \\
\hline H16A & 6030.54 & 5528.46 & 5067.85 & 58 \\
\hline H16B & 6561.24 & 4802.26 & 4936.9 & 58 \\
\hline $\mathrm{H} 16 \mathrm{C}$ & 6109.02 & 5288.76 & 4342.61 & 58 \\
\hline H18 & 5866.08 & 5665.15 & 3179.67 & 27 \\
\hline H19 & 6365.24 & 5248.99 & 2213.63 & 30 \\
\hline $\mathrm{H} 21$ & 8867.04 & 6602.83 & 2352.65 & 36 \\
\hline $\mathrm{H} 22$ & 8365.78 & 7030.53 & 3307.34 & 29 \\
\hline $\mathrm{H} 23$ & 8765.23 & 5446.08 & 1633.11 & 35 \\
\hline $\mathrm{H} 24 \mathrm{~A}$ & 7308.4 & 6444.22 & 981.07 & 64 \\
\hline $\mathrm{H} 24 \mathrm{~B}$ & 8231.15 & 6049.9 & 651.18 & 64 \\
\hline $\mathrm{H} 24 \mathrm{C}$ & 8584.99 & 6562.48 & 1241.06 & 64 \\
\hline $\mathrm{H} 25 \mathrm{~A}$ & 7262.52 & 4680.65 & 1518.95 & 50 \\
\hline $\mathrm{H} 25 \mathrm{~B}$ & 7532 & 4915.25 & 823.18 & 50 \\
\hline
\end{tabular}




\begin{tabular}{|c|c|c|c|c|}
\hline H25C & 6505.41 & 5240.58 & 1112.64 & 50 \\
\hline H27 & 1729.55 & 8412.5 & 4734.69 & 30 \\
\hline H28 & 473.6 & 8800.71 & 3863.81 & 29 \\
\hline H30 & 2442.71 & 8108.96 & 2608.26 & 29 \\
\hline H32 & -78.93 & 9018.14 & 2761.11 & 31 \\
\hline H33A & 1325.48 & 9571.56 & 2303.22 & 51 \\
\hline H33B & 424.55 & 9289.64 & 1736.79 & 51 \\
\hline H33C & 1565.93 & 8891.21 & 1919.64 & 51 \\
\hline H34A & 423.2 & 7806.15 & 2088.24 & 54 \\
\hline H34B & -660.24 & 8259.19 & 1895.65 & 54 \\
\hline H34C & -484.18 & 7855.97 & 2569.18 & 54 \\
\hline H35A & 5754.92 & 7316.94 & 2643.26 & 31 \\
\hline H35B & 4533.97 & 7617.03 & 2478.6 & 31 \\
\hline H36A & $3890(80)$ & $5940(50)$ & $4196(15)$ & $110(30)$ \\
\hline H36B & $4080(60)$ & $5700(30)$ & $3500(30)$ & $70(20)$ \\
\hline H37A & 7671.53 & 8215.39 & 3135.92 & 55 \\
\hline H37B & 7556.8 & 8985.38 & 2874.56 & 55 \\
\hline H37C & 7390.37 & 8359.43 & 2376.48 & 55 \\
\hline H38A & 5157.7 & 8991.03 & 1839.42 & 67 \\
\hline H38B & 5324.68 & 9584.57 & 2371.99 & 67 \\
\hline H38C & 4243.24 & 9116.59 & 2305.16 & 67 \\
\hline H39A & 4867.13 & 8851.5 & 3779.13 & 71 \\
\hline H39B & 5925.4 & 9322.5 & 3743.41 & 71 \\
\hline H39C & 6071.84 & 8566.98 & 4038.28 & 71 \\
\hline H40A & 2312.68 & 6771.31 & 2521.2 & 72 \\
\hline H40B & 1317.66 & 6249.85 & 2319.55 & 72 \\
\hline H40C & 2551.67 & 6031.64 & 2242.49 & 72 \\
\hline H41A & 1324.62 & 6165.46 & 4248.82 & 69 \\
\hline H41B & 518.37 & 6297 & 3589.7 & 69 \\
\hline H41C & 1430.19 & 6854.65 & 3848 & 69 \\
\hline H42A & 2475.1 & 4741.36 & 3126.32 & 68 \\
\hline H42B & 1206.27 & 4955.58 & 2966.23 & 68 \\
\hline H42C & 1745.02 & 4832.73 & 3699.94 & 68 \\
\hline
\end{tabular}

\section{References}

1. Johnson, K. R. D.; Hayes, P. G. Organometallics 2009, 28, 6352.

2. Cantat, T.; Scott, B. L.; Kiplinger, J. L. Chem. Commun. 2010, 46, 919.

3. MacNeil, C. S.; Glynn, K. E.; Hayes, P. G. Organometallics 2018, 37, 3248.

4. Dolomanov, O. V.; Bourhis, J.; Gildea, R. J.; Howard, J. A. K.; Puschmann, H. J. Appl. Cryst. 2009, 42, 339.

5. (a) Sheldrick, G. M. Acta Cryst. 2015, A71, 3.; (b) Sheldrick, G. M. Acta Cryst. 2015, C71, 3. 